Alain MARCHANDISSE

\title{
L'obituaire du chapitre de Saint-Materne à la cathédrale Saint-Lambert de Liège
}

BRUXELLES

PALAIS DES ACADÉMIES

rue Ducale, 1 
Extrait du Bulletin de la Commission royale d'Histoire,

t. CLVII, 1991, pp. 1-124. 


\title{
L'obituaire du chapitre de Saint-Materne à la cathédrale Saint-Lambert de Liège
}

\author{
par Alain MARCHANDISSE \\ Université de Liège
}

\section{AVANT-PROPOS}

Il est de ces objets d'étude qui, pour être importants, ne suscitent que sporadiquement l'intérêt des historiens belges. Tel est précisément le cas des sources nécrologiques. En effet, si elles ont fait l'objet d'un regain d'intérêt à l'étranger (1), force nous est de constater qu'en revanche, dans notre pays, l'édition des obituaires n'a pas connu une efflorescence exceptionnelle, ces dix dernières années (2). Pourtant l'apport des documents nécrologiques est considérable dans tous les domaines de la recherche historique. D'aucuns l'ont bien montré (3). Tant l'histoire économique (4), l'histoire des mentalités, l'histoire de

(1) Pour la bibliographie des ouvrages que les éruditions allemande, française et italienne ont consacré aux documents nécrologiques, on se reportera à N. HuYGHEBAERT, Les documents nécrologiques, TYPOLOGIE DES SOURCES DU MOYEN ÂGE OCCIDENTAL, fasc. 4, Turnhout, 1972, pp. 60-62. - J.-L. Lemaître, Mise à jour de N. HuyghebaerT, Les documents nécrologiques, Turnhout, 1985, pp. 1-10. - Ph. GeORGE, La mémoire des morts, Le MoYen ÂGE, t. 95, 1989, pp. 527-534.

(2) Le séminaire d'histoire du moyen âge de l'Université de Liège s'est toutefois montré assez prolifique en la matière. Plusieurs collaborateurs ou étudiants du professeur A. Joris ont, en effet, consacré publications et mémoires de licence à l'édition de divers obituaires du diocèse de Liège. Cf. J. DeCKers et Chr. Renardy, L'obituaire de la collégiale Notre-Dame de Huy, Bruxelles, 1975. - Cl. MOREAU, L'obituaire de la collégiale Sainte-Ode d'Amay. Édition et commentaires, mém. de lic. en Histoire, dactyl., Université de Liège, Liège, 1982-1983. - E. ClosSET, L'obituaire du Neufmoustier-lez-Huy. Étude des mains médiévales, mém. de lic. en Histoire, dactyl., Université de Liège, Liège, 1987. 1988. - A. Marchandisse, L'obituaire de la cathédrale Saint-Lambert de Liége (XI$X V^{e}$ siècles), Bruxelles, 1991 (sous presse). Cf. également Ph. GeORGE, op. cit., p. 533.

(3) N. HuyghebaerT, op. cit., pp. 63-70. - J.-L. Lemaître, Les obituaires français. Perspectives nouvelles, Revue D'HISTOIRE De L'ÉGLISE DE France, t. 64, 1978, p. 71. L. Génicot, Une source mal connue de revenus paroissiaux : les rentes obituaires. L'exemple de Frizet, Louvain-la-Neuve, 1980.

(4) Les obituaires constituent, par exemple, de précieux compléments des cartulaires pour quiconque entreprend l'étude du domaine des établissements religieux. 
l'art que la prosopographie, l'onomastique voire l'histoire politique, sociale et liturgique peuvent y trouver une matière appréciable (5). Aussi nous a-t-il semblé utile d'éditer un document dont l'on a fait peu de cas par le passé : l'obituaire du chapitre de Saint-Materne, à la cathédrale de Liège (6). Ce collège fut fondé au début du XIII ${ }^{\mathrm{e}}$ siècle, entre autres raisons pour pallier les manquements dont se rendaient coupables les chanoines de Saint-Lambert dans l'exercice de leur ministère (7). Tout comme l'obituaire qui émane de lui, ce chapitre secondaire n'a guère retenu l'attention des historiens (8). Certes, les chanoines de Saint-Materne n'avaient pas le prestigieux statut des chanoines tréfonciers. Il n'en reste pas moins qu'ils s'associaient aux chanoines cathédraux et aux autres bénéficiers - notamment les chanoines de la Petite Table (9) - pour constituer l'un des plus importants chapitres de l'Empire (10).

(5) Il suffira, pour s'en convaincre, de se reporter à l'introduction de notre édition de l'obituaire de Saint-Lambert, mentionnée à la note 2 .

(6) L'édition de l'obituaire de Saint-Materne s'avère intéressante, ne fût-ce que parce qu'elle constitue un complément à l'édition de l'obituaire de Saint-Lambert, mentionnée à la note 2. Elle était d'ailleurs préconisée par dom U. Berlière dans le cadre du programme de publication des obituaires belges décidé par la C.R.H. au début du siècle. Cf. Lettre de dom U. Berlière à G. Kurth, 26 décembre 1904 (cf., à son propos, S. ORLOFF, M. Rucquoy, D. Figa, C. Nó́, G. Vandendosch, Inventaires des Archives de l'académie Royale des Sciences, des Lettres et des Beaux-Arts de Belgique, 1769-1984, sous la dir. de J.-L. DE PAEPE, Bruxelles, 1986, p. 1020, $\mathrm{n}^{\circ} 16.334$ [dossier obituaire]). À propos des projets de la C.R.H. en matière d'obituaires, ef. H. WELLMER, Le nécrologe de la cathédrale Saint-Lambert de Liège, Le Moyen ÂGe, t. 74, 1968, pp. 422-425. - A. MARChandisse, L'obituaire de la cathédrale Saint-Lambert de Liège. Notes pour une édition, LE MOYEN ÂGE, t. 96, 1990, pp. 411-420.

(7) Cf. Introduction, chapitre 1.

(8) À notre connaissance, seules deux études lui ont été consacrées: L. LAHAYE, Les chanoines de Saint-Materne à Saint-Lambert de Liège, B.S.A.H.D.L., t. 27, 1936, pp. 97-150, qui, bien que précieux, pèche par le caractère restreint de sa problématique (cf. introduction, chapitre 5, note 94), et Abbé J. Brouwers, Le Chapitre de SaintMaterne à Liège et Gelinden, Leodium, t. 71, 1986, pp. 1-20, lequel concerne essentiellement l'époque moderne.

(9) Sur ce chapitre qui n'a pas encore fait l'objet d'une étude approfondie, cf. J. GöRRES, Das Lütticher Domkapitel bis zum 14. Jahrhundert, Diss., Berlin, 1907, pp. 15-16. A. DuBors, Le chapitre cathédral de Saint-Lambert à Liège au XVIr siècle, Liège, 1949, pp. 157-158.

(10) Outre les deux ouvrages vieillis de J. DE Theux de Montuardin, Le chapitre de Saint-Lambert à Liège, 4 vol., Bruxelles, 1871-1872 et J. DARIS, Notice sur le Chapitre de Saint-Lambert, à Liège, Notices SUR Les ÉGliSes du DtocèSE DE LiĖGe, t. 3, Louvain, 1872, pp. 145-262, cf., à son propos, S. CHOT-STASSART, Le chapitre cathédral de SaintLambert à Liège au moyen âge. Nationalité, conditions juridique, sociale et intellectuelle des chanoines, mém. de lic. en Histoire, dactyl., Université de Liège, Liège, 1954-1955. Cf. également J. GörRes, op. cit. et A. DUBOIS, op. cit. 
Grâce à l'édition et à l'étude de l'obituaire de Saint-Materne, document conservé sous la forme d'un original, datant de la seconde moitié du XIV $\mathrm{XI}^{\mathrm{e}}$ siècle et jusqu'alors inédit, nous espérons pouvoir apporter une lumière nouvelle sur l'organisation du chapitre cathédral et sur l'histoire du diocèse et de la principauté de Liège au moyen âge.

C'est pour nous un bien agréable devoir de remercier ici MM. les professeurs A. Joris et J.-L. Kupper, respectivement professeur honoraire aux Universités de Liège et de Luxembourg, et chargé de cours aux Universités de Liège et de Luxembourg. Nous leur sommes en effet redevable de très nombreux et judicieux conseils. Tous deux ont également accepté de relire cet article. Nous les en remercions chaleureusement.

Nous tenons également à exprimer notre gratitude à Messieurs Hansotte, Joris, Verhulst et Wyffels, qui ont examiné cette publication pour la Commission royale d'Histoire et l'ont enrichie de leurs suggestions. Nous avons tenté d'en tirer parti au mieux. 


\section{BIBLIOGRAPHIE DES PRINCIPAUX OUVRAGES UTILISÉS ET TABLE DES ABRÉVIATIONS}

\section{REMARQUE LIMINAIRE}

On remarquera que les notices bibliographiques ont été précédées d'une désignation abrégée (généralement nom de l'auteur suivi des premiers mots du titre ou titre de la source et nom de son éditeur). Ces abréviations, réservées aux ouvrages mentionnés à plusieurs reprises dans cet article, visent à alléger l'apparat bibliographique et à améliorer la compréhension du lecteur (11). Afin de faciliter la résolution des abréviations, nous avons classé les ouvrages par ordre alphabétique, sans établir de distinction entre sources (manuscrites et imprimées) et travaux.

A.C.H.S.B.A. : Annales du Cercle hutols des Sciences et BeauX-Arts.

A.É.L. : Archives de l'ÉtAT À Liège.

A.H.E.B. : ANALECTES POUR SERVIR À L'Histoire eCclésiastique de la BelgiQUE.

B.C.R.H. : Bulletin DE LA COMmission Royale D'HistorRe.

BERLIÈre, Instrumenta Miscellanea: U. BERLIÈRE, Inventaire des Instrumenta Miscellanea des Archives Vaticanes au point de vue de nos anciens diocèses, B.I.H.B.R., t. 4, 1924, pp. 6-162.

Berlière, Lettres Clément VI: U. Berlière, Lettres de Clément VI (13421352), t. 1 (1342-1346), Analecta Vaticano-Belgica, t. 6, Rome-Bruxelles-Paris, 1924.

Berlière, Suppliques Clément VI: U. Berlière, Suppliques de Clément VI (1342-1352), Analecta Vaticano-Belgica, t. 1, Rome-Bruges-Paris, 1906.

BerLière, Suppliques Innocent VI: U. Berlière, Suppliques d'Innocent VI (1352-1362), Analecta Vaticano-Belgica, t. 5, Rome-Bruxelles-Paris, 1911.

Bormans, Conclusions capitulaires : St. Bormans, Répertoire chronologique des

(11) Notre souci est de répondre au vœu légitime émis par M.C. WyFfels dans Bibliografieën en renvooien in voethoten in wetenschappelijke publikaties, BIBLIOTHEEK- EN ArCHIEFGIDS, t. 66, no 1, 1990, pp. 131-134. 
conclusions capitulaires du chapitre cathédral de Saint-Lambert, à Liège, Liège, 1869-1875.

Bormans, Saint-Denis: St. Bormans, Notice des cartulaires de la collégiale Saint-Denis à Liège, B.C.R.H., $3^{\mathrm{e}}$ sér., t. 14, 1872, pp. 23-190.

Bormans-SChOOLMEesters, Liber officiorum : St. Bormans, E. SchoolmeEsTERS, Le Liber officiorum ecclesiae Leodiensis, B.C.R.H., $5^{\mathrm{e}}$ sér., t. 6, 1896, pp. 445-520.

B.I.A.L. : BUlletin DE L'INSTITUT ARCHÉOLOGIQUE LIÉGEOIS.

B.I.H.B.R. : BULLETIN DE L'INSTITUT HISTORIQUE BELGE DE ROME.

Brouwers, Saint-Materne et Gelinden: J. Brouwers, Le chapitre de SaintMaterne à Liège et Gelinden, LeODium, t. 71, 1986, pp. 1-20.

C.S.L. : St. Bormans, É. Schoolmeesters, É. Poncelet, Cartulaire de l'Église Saint-Lambert à Liège, 6 vol., Bruxelles, 1893-1933.

B.S.A.H.D.L. : Bulletin de la Société d’Art et D'Histoire du Diocèse de LIÈGE.

B.S.S.L.L. : Bulletin de la Société ScIentifique et litTÉRaire du LiMBOURG.

B.T.D. : Bulletin de la Commission royale de Toponymie et de DialectoLOGIE.

Chot-Stassart : S. Chot-Stassart, Le Chapitre cathédral de Saint-Lambert à Liège au moyen âge. Nationalité, conditions juridique, sociale et intellectuelle des chanoines, mém. de lic. en Histoire, dactyl., Université de Liège, Liège, 1954-1955.

Ob. Neufmoustier, éd. Closset : E. Closset, L'obituaire du Neufmoustier-lezHuy : Étude des mains médiévales, mém. de lic. en Histoire, dactyl., 2 vol., Université de Liège, Liège, 1987-1988.

Ob. Saint-Laurent (Liège), éd. Coens : M. COENS, Un calendrier-obituaire de Saint-Laurent de Liège, Analecta Bollandiana, t. 58, 1940, pp. 48-78.

C.R. : Compte-rendu.

C.R.H. : COMMISSION ROYALE D'HistoIRE.

Cuvelier: Val-Benoit: J. Cuvelier, Cartulaire de l'abbaye du Val-Benoit, Bruxelles, 1906.

Cuvelier, Inventaire Val-Benoît: J. Cuvelier, Inventaire des archives de l'abbaye du Val-Benoît-lez-Liège de l'ordre de Cîteaux, B.I.A.L., t. 30, 1901, pp. 1-706.

Ob. N.-D. Huy, éd. Deckers-Renardy : J. Deckers, Chr. Renardy, L'obituaire de la collégiale Notre-Dame à Huy, Bruxelles, 1975.

De Theux, S.L. : J. De Theux De MontJardin, Le chapitre de Saint-Lambert à Liège, 4 vol., Bruxelles, 1871-1872.

Néc. N.-D. Maestricht, éd. Doppler : P. DOPPLER, Nécrologe de l'église collégiale de Notre-Dame à Maestricht, commencé vers 1307, P.S.H.A.L., t. 52, 1916, pp. 241-315.

Ob. Saint-Servais Maestricht, éd. Doppler : P. DopPler, Obituaire de l'église collégiale, libre et impériale de Saint-Servais à Maestricht commencé vers 1294, P.S.H.A.L., t. 47, 1911, pp. 261-325.

Evrard, Flône: H. Evrard, Documents relatifs à l'abbaye de Flône, Louvain, 1894 (tiré-à-part de A.H.E.B., t. 23-24, 1892-1893).

FaIron, Regestes: Fairon, Regestes de la Cité de Liège, 4 vol., Liège, 19331939. 
FAyen, Lettres Jean XXII: A. FAYen, Lettres de Jean XXII (1316-1334), analecta Vaticano-Belgica, t. 2-3, Rome-Bruxelles-Paris, 1908-1912.

Fierens, Lettres Urbain $V:$ A. Fierens, Lettres d'Urbain $V$ (1362-1370), t. 1 (1362-1366), Analecta Vaticano-Belgica, t. 9, Bruxelles-Rome-Paris, 1928.

Fierens, Suppliques Urbain V: A. Fierens, Suppliques d'Urbain $V$ (13621370), Analecta Vaticano-Belgica, t. 7, Rome-Bruxelles-Paris, 1914.

GENICOT, Les chanoines de Huy: L.-F. GÉNICOT, Les chanoines et le recrutement du chapitre de Huy pendant le moyen âge, A.C.H.S.B.A., t. 27, 19631964, pp. 1-99.

GOBERT, Les rues de liège: Th. GoBert, Liège à travers les âges. Les rues de Liège, $2^{\mathrm{c}}$ éd., 6 vol., Liège, 1924-1929.

GroTEFEND, Zeitrechnung: H. GroTEFEND, Zeitrechnung des deutschen Mittelalters und der Neuzeit, 2 vol., Hanovre, 1891-1892.

Huyghebaert, Documents nécrologiques: N. HuYGHebaert, Les documents nécrologiques, TYPOLOGIE DES SOURCES DU MOYEN ÂGE OCCIDENTAL, t. 4, Turnhout, 1972.

Hemricourt : Jacques de Hemricourt, Euvres, éd. C. DE Borman, A. Bayot, É. Poncelet, 3 vol., Bruxelles, 1910-1931.

Joris, Ville de Huy: A. JORIS, La ville de Huy au moyen âge. Des origines à la fin du $X I V^{n}$ siècle, Paris, 1959.

KUPPER, Liège et l'Église impériale: J.-L. KuPPER, Liège et l'Église impériale ( $X I^{P}$-XII siècles), Paris-Liège, 1981.

LAHAYE, Saint-Jean: L. LAHAYE, Inventaire analytique des chartes de la collégiale de Saint-Jean l'Évangéliste à Liège, 2 vol., Bruxelles, 1921-1931.

Lahaye, Saint-Materne: L. Lahaye, Les chanoines de Saint-Materne à SaintLambert de Liège, B.S.A.H.D.L., t. 27, 1936, pp. 97-150.

Lahaye, Paroisses: L. Lahaye, Les paroisses de Liège, B.I.A.L., t. 46, 1921, pp. 1-208.

LEMA ÎTRE, Répertoire : J.-L. LEMAîTRE, Répertoire des documents nécrologiques français, sous la dir. de P. Marot, RecueIL DES historiens DE LA FranCE, publié par l'Académie des inscriptions et belles-lettres, OBITUAIRES, t. 7, Paris, 1980.

O.S.L., éd. MARChANDISSE: A. MaRChandisSE, L'obituaire de la cathédrale Saint-Lambert de Liège ( $X I^{i}-X V^{r}$ siècles), Bruxelles, 1991 (à paraître).

MoREL, Calendriers perpétuels: E. MOREL, Les calendriers perpétuels en usage dans les diocèses de Beauvais, Noyon et Senlis du XIII siècle au XVII Compiègne, 1911.

M.A. : Le Moyen ÂGE.

Naveau-Poullet : L. Naveau de Marteau, A. Poullet, Recueil d'Épitaphes de Henri van den Berch, héraut d'armes Liège-Looz de 1640 à 1666, 2 vol., Liège, 1925-1928.

Ob. St-Barthélemy: Obituaire, sur parchemin, $\mathrm{XVI}^{e}$ s. avec ajouts, A.É.L., Fonds de la collégiale Saint-Barthélemy, $\mathrm{n}^{\circ} 1$.

Ob. Ste-Croix (I): Stock, $\mathrm{f}^{\circ} 121 \mathrm{r}^{\circ}-143 \mathrm{v}^{\circ}$, Fragment d'un obituaire (jusqu'au 4 novembre), $\mathrm{XV}^{\mathrm{e}}$ s., A.É.L., Fonds de la collégiale Sainte-Croix, $\mathrm{n}^{\circ} 14$.

Ob. Ste-Croix (II) : Obituaire sur parchemin, XV'-XVI', A.É.L., Fonds de la collégiale Sainte-Croix, $\mathrm{n}^{\circ} 101$.

Ob. Ste-Croix (III) : Obituaire sur papier, $\mathrm{XV}^{\mathrm{e}}-\mathrm{XVI}^{\mathrm{e}}$ s., A.É.L., Fonds de la collégiale Sainte-Croix, $\mathrm{n}^{\circ} 102$. 
Ob. St-Denis : Commemorationes fratrum et benefactorum ecclesie sancti Dyonisii, XIII ${ }^{\mathrm{e}}$ s. avec ajouts, A.É.L., Fonds de la collégiale Saint-Denis, $\mathrm{n}^{\circ} 57$.

Ob. Saint-Materne: Obituaire du chapitre de Saint-Materne, sur parchemin, $\mathrm{XIV}^{\mathrm{e}}-\mathrm{XV}^{\mathrm{e}}$ s., A.É.L., Fonds de la cathédrale Saint-Lambert, Chapitre de Saint-Materne, $\mathrm{n}^{\mathrm{o}} 3$.

OEDIGER, Regesten: F. W. OEDIGER, Die Regesten der Erzbischöfe von Köln im Mittelalter, t. 1, 313-1099, Bonn, 1954.

Pairoux, Une vie inédite: A. Pairoux, Une vie inédite de saint Simètre de Lierneux (XIV siècle), B.C.R.H., t. 154, 1988, pp. 199-226.

Paquay, Cartulaire N.-D. Tongres: J. Paquay, Cartulaire de la collégiale Notre-Dame à Tongres jusqu'au $X V^{e}$ siècle, 2 vol., Tongres, 1909.

Ob. N.-D. Tongres, éd. Paquay: J. Paquay, Obituaire de la Collégiale Notre-Dame à Tongres, B.S.S.L.L., t. 40, 1926, pp. 74-79; t. 41, 1927, pp. $66-99$; t. 42,1928 , pp. 67-92.

Paquay, Saint-Barthélemy: J. Paquay, La collégiale Saint-Barthélemy à Liège. Inventaire analytique des chartes, Liège, 1935.

Platelle, Missel de Saint-Amand: H. Platelle, Un missel du $X V^{e}$ siècle à l'usage de l'abbaye de Saint-Amand (ms. Valenciennes $n^{\circ} 118$ ). Le donateur, l'enlumineur, le contenu, dans Littérature et Religion, Mélanges G. Coppin, Lille, 1966, pp. 119-155.

Poncelet, Hugues de Pierrepont : É. Poncelet, Actes de Hugues de Pierrepont (1200-1229), Bruxelles, 1941.

Poncelet, Saint-Pierre: É Ponceler, Inventaire analytique des chartes de la collégiale de Saint-Pierre à Liège, Bruxelles, 1906.

PONCELET, Sainte-Croix: É. PonCELET, Inventaire analytique des chartes de la collégiale de Sainte-Croix à Liège, 2 vol., Bruxelles, 1911-1922.

Poncelet, F.A.M.: É. Poncelet, Le livre des fiefs de l'église de Liège sous Adolphe de la Marck, Bruxelles, 1898.

Poncelet, F.E.M. : É. Poncelet, Les feudataires de la principauté de Liège sous Englebert de la Marck, Bruxelles, 1948.

P.S.S.L.L. : Publications de la Sociéte SCientifique et littéraire du LimBOURG.

R.B.P.H. : Revue belge De Philologie et d'Histoire.

RENARDY, Les maîtres universitaires: Chr. RENARDY, Les maîtres universitaires du diocèse de Liège. Répertoire biographique, 1140-1350, Paris, 1981.

SCHOOLMEesters, Notice: E. SCHOOLMEESTERS, Notice concernant un manuscrit de l'ancienne abbaye de Saint-Jacques à Liège, relatif au schisme de Thierry de Perwez (1406-1408), B.C.R.H., $4^{\mathrm{e}}$ sér., t. 15, 1888, pp. 4-57.

Schoonbroodr, Val-Saint-Lambert: J. G. SCHOONBRoOdr, Inventaire analytique et chronologique des archives de l'abbaye du Val-Saint-Lambert-lezLiège, 2 vol., Liège, 1875-1880.

Schoonbroodt, Saint-Martin: J. G. Schoonbroodt, Inventaire analytique et chronologique des chartes du chapitre de Saint-Martin à Liège, Liège, 1871.

Series episcoporum: Series episcoporum ecclesiae catholicae occidentalis, series V, Germania, t. 1, Archiepiscopatus Coloniensis, Stuttgart, 1982.

Stiennon, Paléographie: J. Stiennon, Paléographie du Moyen Âge, Paris, 1973.

Thimister, Saint-Paul: O. J. Thimister, Cartulaire ou recueil de chartes et documents inédits de l'église collégiale de Saint-Paul actuellement cathédrale de Liège, Liège, 1878. 
TiHon, Lettres Grégoire XI: C. TiHON, Lettres de Grégoire XI (1371-1378), t. 1 ( $\left.n^{\circ} 1-1389\right)$, Analecta Vaticano-Belgica, t. 11, Bruxelles-Rome, 1958 ; t. 2 ( $\left.n^{\circ} 1390-2976\right)$, Analecta Vaticano-Belgica, t. 20, BruxellesRome, 1962 ; t. 3 ( $n^{\circ}$ 2977-4070), Analecta Vaticano-Belgica, t. 25, Bruxelles-Rome, 1964.

TiHon, Lettres Urbain $V$ : C. TiHON, Lettres d'Urbain $V(1362-1370)$, t. 2 (1366-1370), Analecta Vaticano-Belgica, t. 15, Rome-Bruxelles-Paris, 1932.

VAN Der MAde, Guillemins: R. VAN Der MADE, Inventaire analytique et chronologique du chartrier des Guillemins de Liège (1317-1669), Bruxelles, 1955.

Polyptyque de Saint-Lambert: D. VAN DerveEghde, Le polyptyque de $1280 \mathrm{du}$ chapitre de la Cathédrale Saint-Lambert à Liège, Bruxelles, 1958.

VANNÉRUS, Documents: J. VANNÉRUS, Documents relatifs aux conflits ayant surgi, de 1302 à 1310 , entre le comté de Hainaut et l'évêché de Liège, B.C.R.H., t. 72, 1903, pp. 181-304. 


\section{INTRODUCTION}

\section{LE CHAPITRE DE SAINT-MATERNE A SAINT-LAMBERT DE LIËGE}

Conformément aux règles d'édition actuelles en matière de sources nécrologiques (12), il nous paraît nécessaire de retracer brièvement l'histoire du chapitre dont émane le document que nous éditons ici : le chapitre de Saint-Materne, collège secondaire de la Cathédrale de Liège (13).

La fondation du chapitre fit l'objet de deux chartes (14), datées respectivement de 1200 (15) et de décembre 1203 (16). Attachons-nous, tout d'abord, au premier document (17). Par celui-ci, Gauthier de

(12) J.-L. Lemaître, Les obituaires français. Perspectives nouvelles, Revue d'Histolre DE L'ÉGLISE DE FRANCE, t. 64, 1978, p. 79. - ID., Directives pour la préparation d'une édition de document nécrologique, BuLletin PHILOLOGIQUe eT HISTORIQUe, 1979, p. 12.

(13) La présente contribution étant consacrée à l'obituaire de Saint-Materne et non directement à l'établissement religieux auquel il ressortit, on comprendra que nous ne relations pas ici, par le menu, l'histoire du chapitre. Une approche plus détaillée est présentée par LAHAYe, Saint-Materne, pp. 95-150. Nous y renvoyons une fois pour toutes. Cf. également l'exposé de Brouwers, Saint-Materne et Gelinden, pp. 1-20.

(14) Si le second document constitue le seul veritable acte de fondation de SaintMaterne, le premier acte fut un préalable à cette institution, ainsi que nous le verrons dans la suite de cet exposé.

(15) Cf. C.S.L., t. 1, pp. 121-123.

(16) Cf. C.S.L., t. 1, pp. 135-138.

(17) La date de cet acte - 1200, indiction 1 - pose problème et appelle un commentaire. Selon Poncelet, Hugues de Pierrepont, pp. IX-X, le document est faux à la fois par sa forme et par la date qu'il comporte. Afin d'étayer ses dires, il émet trois arguments de valeur inégale, selon nous. Premièrement, l'évêque de Liège, Hugues de Pierrepont, mentionné dans l'acte de 1200 , y est qualifié d'episcopus alors qu'à cette date, il n'est encore qu'electus - il sera consacré le 21 avril 1202 (cf. Renier de Saint-Jacques, Annales, éd. J. Alexandre, Liège, 1874, pp. 65-66). Poncelet pense qu'une confusion entre les termes episcopus et electus est difficilement envisageable dans un document qui a été rédigé à Liège et qui émane du doyen de Saint-Lambert et abbé de Notre-Dame-auxFonts, ce qui constitue une présomption de compétence. Deuxièmement, l'indiction est fausse : c'est l'indiction 3 qui correspond à 1200. Troisièmement, l'acte de 1200 doit être rapproché, chronologiquement, de celui de 1203, dont il sera question dans la suite de notre exposé. En effet, l'acte de 1200 et la première partie de celui de 1203 comportent des listes de témoins pratiquement similaires tant dans la composition que dans l'ordre 
Chauvency, doyen de Saint-Lambert et abbé de Notre-Dame-auxFonts (18), constate, d'une part, que les biens relevant de l'abbatialité de Notre-Dame ont été répartis en une multitude d'infimes prébendes, distribuées sans discernement et, d'autre part, que ses prédécesseurs ne se sont guère souciés de remédier à une situation dont ils ont, au contraire, tiré profit. En conséquence, Gauthier convoque les détenteurs de ces prebendulae, les convainc de renoncer à leur charge (19) et, sur le conseil d'Hugues de Pierrepont, élu de Liège, il ramène à dix le nombre des prébendes de Notre-Dame (20). Il poursuit en définissant les quali-

de citation des témoins. É. Poncelet ne peut concevoir que l'on soit parvenu à réunir les mêmes témoins à trois ans d'intervalle et à propos d'un même sujet. À notre avis, les deux premiers arguments avancés par Poncelet doivent être rejetés. En ce qui concerne la confusion entre episcopus et electus dans l'acte de 1200, il ne faut pas perdre de vue que celui-ci n'est pas un original mais une copie tardive ( $\mathrm{XVI}^{\mathrm{c}}$ siècle), conservée dans le cartulaire de Saint-Materne. C'est peut-être le rédacteur de la copie qui est responsable de la confusion. Quant à l'erreur d'indiction, elle ne prouve qu'une chose : la fragilité de la chronologie de l'acte. Elle n'oblige aucunement à modifier la date de 1200 en 1203. À tout prendre, il faudrait plutôt changer 1200 en 1198 , l'indiction 1 correspondant à 1198 et non à 1203 , ce qui serait une aberration. Pour ce qui est du troisième argument, il est évidemment beaucoup plus troublant et suggère assurément un rapprochement chronologique des deux actes, lesquels seraient, tous deux, de 1203. La remarque de Poncelet est fondée, nous semble-t-il, mais elle n'est confortée par rien. Tout d'abord, l'analyse de la liste des témoins n'invalide pas la date du document 1 (1200). Toutes les personnes citées étaient déjà en fonction à cette date ou avant cette date et le sont d'ailleurs encore après 1203 . On pourrait penser que ce sont les sources narratives qui vont emporter notre conviction. Il n'en est rien. Aucune d'entre elles ne mentionne les faits relatés par les deux actes et, partant, ne précise la chronologie des événements. Quoi qu'il en soit, nous voudrions émettre une hypothèse, qui pourrait expliquer l'identité des deux listes de témoins dans les actes de 1200 et de 1203. L'acte de 1203 comporte deux parties. La première reprend, dans des formulaires quasi identiques, les propos de l'acte de 1200, à savoir la résignation des prébendes mineures de Notre-Dameaux-Fonts. Ceci étant, on a peut-être jugé superflu, en 1203, de donner une nouvelle mouture à une décision prise trois ans auparavant. Peut-être s'est-on contenté de recopier consciencieusement l'acte de 1200, avec sa liste de témoins d'alors. En conséquence, dans l'acte de 1203, seule serait originale, et assortie d'ailleurs d'une autre liste de témoins, tous ecclésiastiques, la seconde partie de l'acte, relative, quant à elle, à l'institution d'un chapitre dit de Saint-Materne et à sa dotation. Mais, tout ceci reste une hypothèse. Dans l'état actuel de nos connaissances, il ne semble pas que l'on puisse apporter une solution péremptoire au problème de chronologie et de diplomatique que pose, à l'évidence, l'acte de 1200 .

(18) Il est commémoré le 24 novembre dans l'ob. Saint-Materne, $\mathrm{f}^{\circ} 46 \mathrm{v}^{\circ}$. Cf. notre édition, p. 101 et n. 385 .

(19) Ceci ne se fera pas sans mal. Entre 1223 et 1229, un clere réclama les revenus de sa prebendula. Il fut débouté de sa demande, dut renoncer définitivement à sa charge mais reçut une compensation pécuniaire de la part des chanoines de Saint-Materne. C. C.S.L., t. 1, pp. 214-215.

(20) Il semble qu'avant 1200 , le chapitre de Notre-Dame comportait vingt canonicats. Cf. Brouwers, Saint-Materne et Gelinden, pp. 2-3. 
tés requises pour accéder à cette charge, le mode de désignation à celleci et l'organisation interne du chapitre. Après avoir souligné l'interdiction de cumuler un canonicat à Saint-Materne avec tout autre bénéfice ecclésiastique liégeois (21), il dote le nouveau chapitre en lui assignant d'importants revenus pris sur le patrimoine de Notre-Dame-aux-Fonts et situés à Villers-l'Évêque et dans ses dépendances (22).

Pour l'essentiel, le second document, daté, quant à lui, de décembre 1203 , corrobore le premier. Il fait état cependant d'une modification importante. En effet, à l'instigation de Guy, évêque de Palestrina et légat pontifical, les dix canonicats institués en 1200 sont détachés de Notre-Dame-aux-Fonts et forment un nouveau chapitre, dépendant de celui de la Cathédrale et placé sous l'invocation de saint Materne (23).

Sa raison d'être répond au vœu d'Hugues de Pierrepont, lequel était d'apporter une solution au relâchement de la vie religieuse liégeoise, notamment dans le chef des tréfonciers (24). Assurer le service du culte, telle sera la tâche première des chanoines de Saint-Materne (25), lesquels sont placés, en dignité, entre les chanoines primaires et les bénéficiers (26). La fondation du nouveau collège est confirmée en 1203 par l'évêque de Liège (27) et par le légat pontifical, Guy, évêque de Palestrina (28).

(21) Cette prescription a pour but de focaliser l'attention des chanoines de SaintMaterne sur ce qui devait constituer leur tâche primordiale : le service du culteà Saint-Lambert. $\mathrm{Cr}$. ci-après.

(22) Cf. C.S.L., t. 1, pp. 121-123.

(23) Cf. C.S.L., t. 1, pp. 135-138.

(24) Cf. Poncelet, Hugues de Pierrepont, pp. XXXIV-XXXIX. Au vu des efforts déployés par l'évêque de Liège pour pallier les incongruités de la vie religieuse liégeoise, efforts d'ailleurs soulignés par Poncelet lui-même, nous pensons que l'acte de fondation du chapitre de Saint-Materne ne peut être tenu pour un « acte d'importance médiocre " comme Ponchlet l'affirme dans Hugues de Pierrepont, p. X. Cf. également É. Poncelet, La cessation de la vie commune dans les églises canoniales de Liège, ANNUAIRE D'HISTOIRE LIÉGEOISE, t. 4, 1952, pp. 613-648.

(25) Signalons que le 25 février 1303, le pape Boniface VIII mit l'accent sur cette obligation. Tout chanoine qui contreviendrait à ses devoirs est passible d'une sentence pécuniaire et ecclésiastique. Cf. C.S.L., t. 3, p. 33 ; t. 6, p. 44. Cf. également LaHAYE, Saint-Materne, pp. 102-103.

(26) Cf. C.S.L., t. 1, pp. 121-123, 135-138. Nous entendons par bénéficiers les simples désservants des multiples autels de la Cathédrale. À leur propos, cf. A. DuBors, Le chapitre cathédral de Saint-Lambert à Liège au XVIT siècle, Liège, 1949, pp. 158-160.

(27) $\mathrm{Cr}$. Poncelet, Hugues de Pierrepont, p. 12. Ca 1215, il confirme ses possessions au chapitre de Saint-Materne. Cf. C.S.L., t. 1, pp. 181-182. - PonCelet, Hugues de Pierrepont, pp. 140-141.

(28) Cf. C.S.L., t. 1, pp. 123-124, 138-139. Guy de Palestrina confirmera également une autre fondation de Gauthier de Chauvency : celle de l'hôpital de Saint-Mathieu-à-la-chaîne. Cf. C.S.L., t. 1, pp. 139-140. 
Que le chapitre nouvellement créé ait pris pour patron saint Materne n'a rien d'étonnant. Ce personnage, considéré au moyen âge comme un disciple de saint Pierre, dont l'historicité est prouvée (29) mais dont il est difficile d'établir avec précision les éléments de la biographie (30), a été particulièrement honoré en Belgique. La fondation de nombreuses églises de notre pays lui a été attribuée. Ainsi à Huy (31), à Tongres ou à Namur. Il fut choisi comme patron principal de diverses églises du diocèse de Namur et comme patron secondaire à la collégiale de Tongres. Disons pour faire bref que son culte était particulièrement vivace dans les diocèses de Liège, Namur, Trèves et Cologne, notamment (32). Après cette brève parenthèse, reprenons succinctement l'historique du chapitre de Saint-Materne.

En 1223, le chapitre sera augmenté d'un onzième canonicat. Il est fondé par Jean, prévôt de Saint-Lambert (33), et doté de l'église de Gelinden et de ses dépendances (34). C'est le 16 novembre 1274 que sont publiés les statuts de Saint-Materne, lesquels décrivent notamment la composition du collège. Il comporte quatre prébendes presbytérales, quatre diaconales et trois sous-diaconales (35). En 1277, dans la confirmation des statuts par le pape Jean XXI, il est question de douze cano-

(29) Il participa au concile de Rome en 313 et au concile d'Arles en 314. Cf. OEDIGER, Regesten, t. 1, pp. 1-10. - ID., Geschichte des Erzbistums Köln, t. 1, Cologne, 1964, pp. 36-42. -- ID., Das Bistum Köln von den Anfängen bis zum Ende des 12. Jahrhunderts, Cologne, 1972, pp. 23-25. - Series episcoporum, p. 7. - Palroux, Une vie inédite, pp. 202-203, 219 et n. 84. - P. STINTZI, art. Maternus von Köln, LEXIKON DER CHRISTLICHEN IKonographIE, t. 7, Rome-Fribourg-Bâle-Vienne, 1974, col. 585-586. - A.-M. BURG, La Christianisation de l'Alsace et de l'Église de Trèves, ArChives DE L'ÉGlise D'Alsace, t. 24, 1957, pp. 1-20 et, en particulier, pp. 10-11.

(30) Si la qualité d'évêque de Cologne lui est reconnue, en revanche, celle d'évêque de Trèves est l'objet de nombrcuses controverses. Cf. PaIroux, Une vie inédite, p. 219 n. 84. Écrire l'histoire de saint Materne est d'autant plus difficile qu'aux éléments proprement historiques se mêle la légende. Cf. Oediger, Regesten, pp. 1-9. - F. Rousseau, La légende de saint Materne et du dieu Nam à Namur, dans ID., À travers l'histoire de Namur, du Namurois et de la Wallonie. Recueil d'articles de F. Rousseau publié à l'occasion de son nonantième anniversaire, Bruxelles, 1977, pp. 29-69 (repr. anastatique de ANNALES DE LA SOCIÉTÉ ARCHÉOLOGIQUE DE NAMUR, t. 35, 1922, pp. 191-221).

(31) Cf. Joris, Ville de Huy, pp. 70, 71, 79, 111, 116, 188, 481.

(32) Cf. É. De Moreau, Histoire de l'Église en Belgique, des origines au début du $X I I^{e}$ siècle, t. 1, Bruxelles, 1945, pp. 27-29.

(33) Cf. C.S.L., t. 1, pp. 201-202. L'acte sera confirmé en septembre 1223 par Hugues de Pierrepont et le chapitre de Saint-Lambert (cf. C.S.L., t. 1, p. 203. - PONCELET, Hugues de Pierrepont, p. 204), par Conrad, évêque de Porto, légat apostolique, en juin 1224 (cf. C.S.L., t. 1, pp. 208-209).

(34) Cf. Brouwers, Saint-Materne et Gelinden, pp. 1-20.

(35) Cf. C.S.L., t. 2, p. 229. L'acte prescrit également la résidence et interdit le cumul. 
nicats (36). La douzième prébende n'étant jamais plus mentionnée par la suite, on doit conclure soit qu'elle n'a été concédée que durant peu de temps, soit que la mention qui en est faite dans la confirmation pontificale est erronée.

Sans vouloir entrer dans le détail de l'organisation du chapitre, il convient de préciser que la nomination des chanoines de Saint-Materne qui, durant un temps, appartint à l'abbé de Notre-Dame (37), fut transférée au prévôt de Saint-Lambert lorsque l'abbatialité et la prévôté fusionnèrent, le 23 mai 1230 (38). Juridiquement, les chanoines de Saint-Materne relevaient du doyen et du chapitre cathédral (39).

Avant d'en venir au domaine et aux revenus du chapitre de SaintMaterne, il convient encore de signaler que le 16 avril 1315 , le chapitre cathédral concéda aux prébendiers un lieu de réunion - la chapelle dite du nouveau portail - où ils pouvaient désormais accomplir toutes les tâches incombant à un collège de chanoines (40). Par ailleurs, le 2 septembre 1325, Evrard, abbé du monastère de Saint-Mathias, à Trèves, concéda aux bénéficiers de Saint-Materne une relique de leur saint patron (41).

Venons-en, pour terminer, au patrimoine de Saint-Materne. À la dotation primitive de Villers-l'Évêque (42), localité où le chapitre acquit de très nombreux biens et qui fit l'objet de multiples tractations, contestations et arbitrages (43), s'ajoutèrent, dès 1220, deux maisons, en Gérardrie, à Liège, cédées contre redevance par le chapitre de Saint-

(36) Cf. C.S.L., t. 2, pp. 283-284.

(37) Cf. C.S.L., t. 1, pp. 121-123, 135-138.

(38) Cf. acte de l'évêque de Liège Jean d'Eppes (1229-1238), à cette date (cf. C.S.L., t. 1, pp. 267-269. - F. LecomTE, Catalogue des actes de Jean d'Eppes, prince-évêque de Liège (1229-1238), mém. de lic. en Histoire, dactyl., Université de Liège, Liège, 1960-1961, ${ }^{\circ}{ }^{14}$ ), confirmé par le pape Grégoire IX, le 23 décembre 1230 (cf. C.S.L., t. 1, p. 291). À propos de la fusion des deux charges, cf. Lahaye, Paroisses, pp. 68-70. Le droit de nomination des chanoines de Saint-Materne détenu par le prévôt de la Cathédrale, connut diverses restrictions. Cr. LAHAYE, Saint-Materne, p. 101. - Brouwers, Saint-Materne et Gelinden, p. 4 et n. 8. - A. Dubors, Le chapitre cathédral de Saint-Lambert à Liège au XVII siècle, Liège, 1949 , p. 157 et n. 3.

(39) Cf. note 36. Cette juridiction est confirmée par le pape Grégoire IX, le 28 mars 1230 ou 1231. Cf. C.S.L., t. 1, p. 261 ; t. 6, p. 10.

(40) Cf. C.S.L., t. 3, pp. 151-152.

(41) Cf. C.S.L., t. 3, pp. 279-280.

(42) À celle-ci, l'archidiacre Ludolphe (Lydulphe) ajouta, en 1215, les revenus de l'église du lieu. Cf. C.S.L., t. 1, p. 180. À propos de Lydulphe, archidiacre de Hesbaye, cf. ChOT-STASSART, p. 42.

(43) Cf. C.S.L., t. 1, pp. 230, 305, 330-332, 340-341; t. 2, pp. 26-27, 72-73, 236-237, $463-464$; t. 3, pp. $422-424$; t. 4, pp. 509-512, 553-554 ; t. 5, pp. 16, 18, 87, 115, 191, 197, 206, 228 ; t. 6 , pp. 157-158, 165, 170-175. 
Lambert (44). À ces maisons, pour la réfection desquelles les fidèles accordaient souvent une part de leur fondation obituaire (45), se substituèrent, à la fin du XIV ${ }^{\mathrm{e}}$ siècle, trois maisons qui furent endommagées ou détruites en 1468. Elles furent réparées et firent toujours partie du patrimoine de Saint-Materne, même s'il fut parfois question de les vendre (46). En terme de donation, mentionnons encore celle de 1223 : le prévôt de Saint-Lambert, Jean d'Eppes, fonde un onzième canonicat et le dote de l'église de Gelinden (47). D'un point de vue général, on peut dire que le patrimoine de Saint-Materne s'est accru, au fil du temps, en terres et en revenus de toutes sortes (48). Ceux-ci provenaient de biens fonciers, de dîmes, de rentes, de cens (49) et bien évidemment des fondations destinées à assurer la commémoration des fidèles, inscrites dans le livre des morts.

\section{DESCRIPTION DU MANUSCRIT}

1) Support: parchemin d'épaisseur variable.

2) Cahiers et reliure: 7 cahiers qui comportaient, initialement, 8 folios chacun. En raison de la perte de plusieurs folios, le manuscrit compte aujourd'hui : $2 \mathrm{f}^{\circ}$ initiaux $+6 \mathrm{f}^{\circ}+8 \mathrm{f}^{\circ}+8 \mathrm{f}^{\circ}+8 \mathrm{f}^{\circ}+5 \mathrm{f}^{\circ}+$ $3 \mathrm{f}^{\circ}$ ne formant pas cahier et permettant d'achever l'obituaire de SaintMaterne $+2 \mathrm{f}^{\circ}$ additionnels, lesquels constitueraient le fragment d'un obituaire de l'abbaye de Saint-Jacques à Liège (cf. les paragraphes 3 et 10 de cette description). Le verso du dernier folio de chaque cahier présente une signature. Il s'agit du numéro du cahier, rubriqué et en chiffres romains. Les 3 folios qui terminent l'obituaire de Saint-Materne

(44) Cf. C.S.L., t. 1, p. 186.

(45) Cf. ob. Saint-Materne, $\mathrm{f}^{\circ} 40 \mathrm{v}^{\circ}-41 \mathrm{r}^{\circ}$

(46) Cf. C.S.L., t. 4, pp. 508, 553-554. - LaHAYe, Saint-Materne, pp. 108-109. Polyptyque de Saint-Lambert, p. 180.

(47) Cf. ci-avant, p. 12. Le 16 novembre 1224, le pape Honorius III confirme les possessions du chapitre de Saint-Materne (les églises de Gelinden et de Villers-l'Évêque). Cf. C.S.L., t. 1, pp. 211-212.

(48) On trouvera la liste des localités où sont situés les biens de Saint-Materne dans LaHaYe, Saint-Materne, p. 108. Doivent y être ajoutés les lieux mentionnés dans l'ob. SaintMaterne. Bien que nous ne possédions guère de point de comparaison, il nous a semblé que le domaine de Saint-Materne était relativement important, supérieur, en tout cas, à ce que l'on était en droit d'attendre d'un chapitre, somme toute, secondaire.

(49) Cf. C.S.L., t. 1, pp. 197, 284-286 ; t. 2, pp. 382-384, 388, 514-515; t. 3, pp. 187, 393, 423-424; t. 4, pp. $375-376,393,444,497,498,589-590$; t. 5 , pp. $2,15,19,20,98$; t. 6 , pp. $158,160,163,164-165,171-175,177,178,181,187$. 
portent des signatures : ai, aii et aiii $-\mathrm{a} 1$, a2 et a4, le folio a 3 étant manquant. Reliure : plats (ca $283 \times 190 \mathrm{~mm}$ ), en bois, recouverts de cuir brun, gaufré et décoré de losanges; le cuir recouvrait en partie le revers du plat supérieur et l'avers du plat inférieur. Il subsiste encore de façon fragmentaire. Là où le cuir a disparu, le bois est à nu et comporte des taches (d'encre, probablement). Sur le revers du plat supérieur et sur l'avers du plat inférieur apparaissent quatre encoches (ca $25 \mathrm{~mm}$ de long) où est insérée une double corde de chanvre qui assure la solidité de la reliure. Le revers du plat inférieur comporte une boucle de métal (cuivre?), clouée en son centre sur le côté extérieur du plat. Elle emprisonne une languette de cuir blanchâtre (ca $240 \mathrm{~mm}$ ), qui comporte, en son centre, de part et d'autre, deux plaques métalliques (cuivre ?) fixées grâce à quatre clous et percées d'un trou. Une petite plaquette métallique, qui ne tient plus très bien, enferme son extrémité, de part et d'autre. Le trou que comporte la languette vient s'adapter sur l'avers du plat supérieur, sur lequel est fixé une petite pointe métallique et son support horizontal décoré. Ce dispositif assure la fermeture du manuscrit. Dos (ca $262 \times 52 \mathrm{~mm}$ ), probablement en cuir, renforcé par une bande de papier vert, elle-même surmontée d'une autre, de couleur beige, laquelle est couronnée, sur le haut du dos, par une étiquette avec l'inscription suivante : A.É.L. / Cathédrale Saint-Lambert / Chapitre Saint-Materne / 3.

3) Composition : - 1 petit folio (ca $263 \times 52 \mathrm{~mm}$ ), non folioté, couvert d'une écriture du $\mathrm{XV}^{\mathrm{e}}$ siècle et servant probablement à la consolidation de la reliure. Il présente des trous et des taches d'encre;

-2 folios initiaux $\left(1-2 v^{0}\right)$ : le folio $1 r^{\circ}$ comporte des indications de nature comptable datant du XVIII ${ }^{e}$ siècle et une mention d'inventaire au crayon bleu (chapitre Saint-Materne, 3); sur les folios $1 \mathrm{v}^{\circ}-2 \mathrm{r}^{\circ}$ est transcrite une charte rédigée probablement au $\mathrm{XV}^{\mathrm{e}}$ siècle et dont la lecture est rendue difficile en raison de la mauvaise conservation du parchemin (souillé, taché, encre ternie) et d'une reliure trop serrée ; le folio $2 \mathrm{v}^{0}$ comporte des indications de nature comptable datant du XVIII ${ }^{\mathrm{e}}$ siècle ;

- $f^{0} 3-51 v^{0}$ : obituaire de Saint-Materne, avec lacunes. Le texte complet de l'obituaire devait être rédigé sur 7 cahiers de 8 folios, auxquels s'ajoutaient 4 folios additionnels, soit 60 folios. Seuls 49 folios subsistent. Les lacunes ( 11 folios) concernent les périodes allant du 13 au 24 janvier, du 25 juillet au 12 août, du 7 septembre au 6 octobre et du 13 au 18 décembre. Les folios manquants doivent avoir été perdus après 1936. En effet, l'article de LAHAYE, Saint- 
Materne, p. 111, datant de 1936, mentionne l'existence d'un chanoine Arnold de Brouckem, commémoré le 23 janvier dans l'ob. SaintMaterne. Le folio qui comportait la ou les notice(s) du 23 janvier est aujourd'hui perdu ;

- $\mathrm{f}^{\circ} 52-53 \mathrm{v}^{0}$ : fragment d'un obituaire de l'abbaye de Saint-Jacques de Liège (?). Cf. bibliographie du manuscrit, paragraphe 10 ;

- 1 folio semblable au premier (ca $258 \times$ $53 \mathrm{~mm}$ ), plié et taché, avec indications comptables modernes, au verso.

4) Nombre et numérotation des folios : 53 folios, foliotés au crayon noir dans le coin supérieur droit par une main du $\mathrm{XIX}^{\mathrm{e}}$ siècle. Celle-ci a également numéroté, à gauche de chaque page, les jours selon le calendrier actuel, a indiqué, au-dessus du recto et du verso de chaque folio, le mois dont relèvent les notices rédigées plus bas, et a mentionné, au bas des commémorations de certains chanoines, la date de leur décès.

5) Dimensions des folios: variable. Approximativement, hauteur à gauche : $265 \mathrm{~mm}$, à droite : $268 \mathrm{~mm}$; largeur en haut : $197 \mathrm{~mm}$, en bas : $190 \mathrm{~mm}$.

6) Présentation et disposition des folios : piqûres : absentes, en ce qui concerne l'obituaire de Saint-Materne proprement dit. En revanche, les deux folios de l'obituaire de Saint-Jacques comportent des piqûres, sur le côté intérieur des folios; réglure d'encadrement à la mine de plomb (marges au recto, à gauche : ca $17 \mathrm{~mm}$, à droite: ca $22 \mathrm{~mm}$, en haut : ca $15 \mathrm{~mm}$, en bas : ca $38 \mathrm{~mm}$ ). Chaque face est divisée en six colonnes de ca $7,5,5,8,5,5,5,2,5$ et $125 \mathrm{~mm}$. La justification est de ca $155 \times 212 \mathrm{~mm}$. Réglures linéaires à la mine de plomb (nombre de lignes/page : 31 ; espace interlinéaire : ca $7 \mathrm{~mm}$ ). Présentation du texte: il comporte un calendrier romain, des lettres dominicales, des nombres d'or et un calendrier liturgique (cf. chapitre 4 de l'introduction). Sont rubriqués : les indications de jours et de lunes en début de mois, l'initiale $\mathrm{K}$ de l'abréviation $\mathrm{KL}$ de kalendas, uniquement le premier du mois, jour des kalendes (la majuscule L est de couleur bleue), les lettres dominicales, certaines fêtes du calendrier liturgique, le degré de solennité (duplex, III lectiones, collecta...) de toutes les fêtes, l'indication, à la fin de chaque mois, du nombre d'heures de clarté et d'obscurité que comptent les jours de chaque mois. Pour ce qui est de la couche initiale, est également rubriquée : l'initiale de l'abréviation de commemoratio et du prénom de la personne commémorée, parfois du nom et de la 
dignité de cette même personne. Par ailleurs, toujours dans la couche initiale et au début de nombreuses commémorations, on constate la présence de pieds de mouche (de couleur bleue ou rouge). Chaque folio comporte les commémorations de trois jours de calendrier, hormis les $\mathrm{f}^{\circ} 10 \mathrm{r}^{\mathrm{O}}-\mathrm{v}^{\mathrm{o}}$ comportant deux jours du calendrier (du 25 au 28 février) et les $f^{\circ} 5 v^{\circ}, 15 v^{\circ}, 25 v^{\circ}, 37 v^{0}, 42 v^{\circ}, 47 v^{0}, 51 v^{0}$, comportant, chacun, quatre groupes de commémorations. Le texte est entièrement rédigé en latin.

7) Écritures et encres : cf. Introduction, chapitre 3.

8) Détériorations : Pour l'essentiel, le document, par ailleurs assez luxueux, est bien conservé. On constate néanmoins que les folios de parchemin sont tannés, surtout dans leur moitié inférieure. La plupart d'entre eux gondolent; certains présentent des plis horizontaux ( $\left.f^{\circ} 44\right)$ ou verticaux $\left(\mathrm{f}^{\mathrm{o}} 45,47\right)$; d'autres sont quelque peu déchirés sur le côté intérieur $\left(\mathrm{f}^{\mathrm{o}} 49\right)$ ou sont détachés, partiellement — vers le bas - $\left(\mathrm{f}^{\circ} 50\right)$ ou complètement $\left(f^{\circ} 39,51\right)$.

9) Conservation actuelle: ARCHIVES DE L'ÉTAT À LiÈGE (A.É.L.), Fonds de la cathédrale Saint-Lambert, Chapitre de Saint-Materne, $\mathrm{n}^{\circ} 3$.

10) Bibliographie du manuscrit (non exhaustive): A.É.L., Inventaire. Cathédrale et archives ecclésiastiques d'ancien régime. Chapitre Saint-Materne, p. 1, $\mathrm{n}^{\circ} 3$ [obituaire, $\mathrm{XV}^{\mathrm{e}}$ siècle] (50). - U. BERLIÈRE, Inventaire des obituaires belges (collégiales et maisons religieuses), Bruxelles, 1899, pp. 40-41:- LaHAYE, Saint-Materne, p. 106 et passim. - Brouwers, Saint-Materne et Gelinden, p. 9 n. 36 . L'obituaire de Saint-Materne est également mentionné dans les deux éditions des folios $52-53 \mathrm{v}^{0}$, lesquels sont présentés comme des fragments d'un obituaire de l'abbaye de Saint-Jacques à Liège : Fragment d'un obituaire du $X I I^{e}$ siècle, éd. St. Bormans, B.I.A.L., t. 9, 1868, p. 511. - Fragments d'un nécrologe de l'abbaye de Saint-Jacques à Liège, éd. U. BERLIÈrE, B.C.R.H., t. 95, 1931, pp. 223-235.

\section{RÉDACTION ET CHRONOLOGIE DU DOCUMENT}

Avant d'aborder le problème de la chronologie de notre document, il est utile, pensons-nous, de revenir sur le problème de la distinction

(50) A. Hansay, Inventaire sommaire du chapitre de Saint-Lambert, Bruxelles, 1902, p. 12, intégré dans cet inventaire, mentionne l'existence d'un obituaire de Saint-Materne, sur parchemin, datant du XIII ${ }^{\mathrm{e}}$ siècle. 
entre nécrologe et obituaire, d'autant que l'obituaire de Saint-Materne apporte des éléments de nature à confirmer l'opinion que nous avons en la matière. À ce propos se sont prononcés deux éminents spécialistes des documents nécrologiques : dom N. Huyghebaert et J.-L. Lemaître. En bref, rappelons que pour dom Huyghebaert, le nécrologe est un livre liturgique stricto sensu, une liste de défunts dont le souvenir est rappelé après l'office de Prime tandis que l'obituaire est une sorte d'aidemémoire à caractère économique, dans lequel sont notées les commémorations fondées (obits) et tout ce qui touche à ces fondations (51). J.-L. Lemaître, quant à lui, distingue nécrologe et obituaire en tenant davantage compte du mode d'inscription que d'un usage liturgique ou non du manuscrit. Selon lui, dans le nécrologe sont inscrits les membres de la communauté religieuse au sens large et tous ceux, bienfaiteurs ou autres, qui sont admis dans sa familiarité, dans la confraternité de prières. L'obituaire, en revanche, renferme le nom des défunts, internes ou externes à la communauté, qui ont demandé la célébration d'un anniversaire et en ont assuré l'exécution par une fondation, dont la nature est variable. Le premier comporte un calendrier, les nom et qualités du défunt, éventuellement une mention d'origine, de legs ou d'officium plenum. Dans le second, on retrouve le calendrier, nom et qualités du défunt, auxquels s'ajoute une fondation plus ou moins détaillée. De l'obituaire disparaissent progressivement, à partir du XIII' siècle, les membres de la communauté religieuse à laquelle appartient le document, au profit des laïcs (52).

Qu'en est-il du document que nous éditons ici ? S'agit-il d'un nécrologe ? S'agit-il d'un obituaire? Tenter de le placer dans une catégorie plutôt que dans une autre, c'est se heurter à des difficultés dans la mesure où il comporte des caractères propres à chacune des deux catégories. Il est en effet à la fois un manuscrit liturgique, ne fût-ce que par la présence d'un calendrier sanctoral, élément liturgique s'il en fut (53), et un registre de fondations, parfois décrites avec force détails. La distinction émise par $\mathrm{N}$. Huyghebaert est donc passablement théorique (54). Quant à celle préconisée par J.-L. Lemaître, bien que mieux assurée, elle suscite quelques difficultés lorsqu'on l'applique au livre des

(51) Huyghebaert, Documents nécrologiques, pp. 33-37.

(52) Lema ÎTRE, Répertoire, pp. 14-26 et en particulier, pp. 24-26. - ID., Mise à jour de N. HuyghebaerT, Les documents nécrologiques; Turnhout, 1985, p. 11.

(53) On trouve également d'autres indications à caractère liturgique. Cf. ob. SaintMaterne, $\mathrm{f}^{\circ} 15 \mathrm{v}^{\circ}$ : Notum sit quod in die palmarum non fiunt vigilie.

(54) Lema ÎTRE, Répertoire, p. 17, souligne que "la distinction entre nécrologe, livre liturgique, et obituaire, livre comptable, est difficile à faire dans la pratique ». 
morts de Saint-Materne. Nous remarquons en effet que notre document comporte des notices à la fois de type «nécrologique » (nom et qualités du défunt, brève mention d'un legs) et de type « obituaire » (nom et qualités du défunt, fondation plus ou moins détaillée de son anniversaire) (55). Ces dernières sont, certes, plus nombreuses et l'on peut penser que notre document ressortit davantage à la classe des obituaires qu'à celle des nécrologes mais il s'agit alors d'un obituaire atypique. En effet, bien loin d'en être exclus au profit des laïcs, les membres de la communauté religieuse, c'est-à-dire les chanoines de SaintMaterne, représentent la moitié des personnes commémorées (67 sur 135). Les laïcs sont, quant à eux, très peu nombreux (56). On le voit, ce que nous appelons « obituaire de Saint-Materne », par habitude liégeoise, est rétif à des critères de classification stricts (57).

$\mathrm{Au}$ vrai, nous pensons qu'il convient d'émettre deux remarques à propos des sources nécrologiques.

Tout d'abord, lorsque l'on aborde un tel document, il faut garder présent à l'esprit qu'un livre des morts est avant tout une source vivante (58), une source qui change, qui évolue au fil du temps, tant dans sa forme que dans sa finalité. De livre liturgique qu'il est aujour-

(55) L'association de notices nécrologiques et obituaires apparaît de façon encore plus éclatante dans l'obituaire de Saint-Lambert, éd. MarChandisse, C.R.H., Bruxelles, 1991 (sous presse).

(56) On peut penser que la forte concentration en chanoines de Saint-Materne s'explique par le fait que ce chapitre est d'importance secondaire, donc moins attractif. Il reste que, pour l'obituaire de Saint-Lambert, document nécrologique émanant d'un chapitre on ne peut plus important et rayonnant, nous avons fait les mêmes constatations. C'est dans le fonds primitif du document, qui correspond davantage à la définition du nécrologe, que l'on trouve les laïcs de condition supérieure (empereurs, rois, ducs, comtes). Bien loin de se multiplier dans ce qui peut être défini comme étant la partie obituaire du document, ils sont au contraire complètement évincés par les dignitaires et les chanoines cathédraux. $\mathrm{Cf}$. O.S.L., éd. MarChandisSE, p. LXII.

(57) Nous avons montré ailleurs que l'identité ou l'altérité de la date d'inscription dans le document nécrologique et de la date de décès ne pouvait constituer un critère infaillible dans la distinction entre nécrologe et obituaire. Il a en effet été souligné que, dans le cas d'un nécrologe, la date d'inscription et la date de décès pouvaient coïncider et qu'en revanche, dans un obituaire, la date de commémoration est sujette à caution (cf. LEMAîtTRE, Répertoire, pp. 76-80. - ID., Prosopographie nobiliaire et documents nécrologiques en Limousin au Moyen Âge, BULLETIN PHLLLOGIQUE ET HISTORIQUE, 1977, pp. 30-31). Il apparaît cependant que, dans le cas de l'obituaire de Saint-Lambert (éd. MaRCHANDISSE, pp. LXvLXVI), la fiabilité de la date d'inscription est grande, tant dans ce que, suivant là les critères de J.-L. Lemaître, nous avons pu détecter comme étant la partie "nécrologe" de notre document que dans sa partie « obituaire».

(58) L'expression est de J.-L. Lemaître dans Un livre vivant, l'obituaire dans Le livre au moyen âge, sous la dir. de J. GLéNIsson, Turnhout, 1988, pp. 92-94. 
d'hui, il sera peut-être registre de la pratique quotidienne d'une administration, demain ou dans d'autres circonstances, tout en conservant, peut-être, une fonction liturgique. Aux notices brèves (« nécrologiques ») qu'il reçoit durant un temps, peuvent venir s'ajouter des commémorations plus étoffées ( obituaires »), ultérieurement. Un document nécrologique est donc difficilement justiciable d'une appellation plutôt que et à l'exclusion d'une autre.

Par ailleurs, vouloir distinguer à tout prix le nécrologe de l'obituaire, c'est en quelque sorte appliquer nos modes de raisonnement cartésiens à des hommes qui pensent différemment de nous, qui interprètent la confusion des choses comme une expression de leur richesse (59), qui considèrent un livre des morts comme un instrument fonctionnel, dont on fait usage tous les jours, comme un "florilège " dans lequel on doit pouvoir trouver, à tout moment, tout ce dont on a besoin : le saint du jour, le nom de la personne pour laquelle il faut prier ou dont il faut célébrer le souvenir, l'origine de telle parcelle de terre, de telle redevance...

Certes, nous sommes conscient du fait que, dans des cas bien précis, il est possible de dire d'un document qu'il est un nécrologe stricto sensu ou un obituaire stricto sensu mais nous pensons qu'il est outrancier de vouloir établir une classification systématique parmi des documents tout à fait singuliers et qui ne peuvent être jugés qu'au cas par cas (60).

Après ces quelques réflexions personnelles, évoquons à présent la chronologie de l'obituaire de Saint-Materne.

Il se présente sous la forme d'un original dont il n'existe, à notre connaissance, aucune copie.

Afin de fixer la date à laquelle commença la commémoration des

(59) On aura une idée de la richesse, de la complexité et en même temps de la confusion des idées des médiévaux dans les schémas de pensée d'un Joachim de Flore ou d'un Ubertin de Casale. 11 s'agit là, certes, de cas extrêmes, qu'il ne faut en aucun cas ériger en absolu. Il n'empêche qu'ils illustrent bien une certaine tendance du temps. À leur propos, cf. la bibliographie établie par M. REEvES, W. Gould, Joachim of Fiore and the myth of the eternal evangel in the nineteenth century, Oxford, 1987, p. 7 n. 1 et, en particulier, M. REEves, The Influence of Prophecy in the later Middle Ages, Oxford, 1969.

(60) À cet égard, l'érudition allemande se montre souvent plus circonspecte en utilisant plutôt le terme Totenbuch (cf. par exemple, G. Althoff, J. Wollasch, Die Totenbücher von Merseburg, Magdeburg and Lünebourg, M.G.H., Libri Memoriales et Necrologia, nova series, t. 2, Hanovre, 1983). L'appellation " livre des morts » est, somme toute, plus proche de la réalité compte tenu du fait que, souvent, les médiévaux ne donnaient pas de titre à leur manuscrit - c'est notamment le cas pour le chapitre de Saint-Materne - ou l'appelaient tout simplement Liber (le mot « obituaire " n'apparait pas avant la fin du moyen âge, le mot " nécrologe" avant le milieu du XVII ${ }^{\mathfrak{e}}$ siècle !). Cf. J.-L. LeMAître, Mise à jour de N. HUYGHEBAERT, Les documents nécrologiques; Turnhout, 1985, p. 11. 
défunts à Saint-Materne, il convient, tout d'abord, d'étudier la couche initiale du document, c'est-à-dire, logiquement, la main qui est responsable de la mise en œuvre du manuscrit. Celle-ci est facilement reconnaissable. En effet, on lui doit à la fois le calendrier sanctoral et une première couche de commémorations qui comportent, pour la plupart, des initiales rubriquées (commemoratio, nom et parfois qualité du défunt). L'écriture présente toutes les caractéristiques de la littera textualis formata (lettre de forme). Il s'agit, en effet, d'une élégante écriture droite, stylisée, aux hampes et aux hastes peu développées et aux pleins bien accentués, le tout à l'encre noire (61). De prime abord, compte tenu du fait qu'elle a traversé tout le bas moyen âge, cette écriture semble pouvoir être datée du $\mathrm{XIV}^{\mathrm{e}}$ ou du $\mathrm{XV}^{\mathrm{e}}$ siècle. Il est toutefois possible de préciser cette date et, partant, d'apporter quelques éclaircissements quant à la nature du document nécrologique. En effet, le cartulaire de SaintMaterne (XVI ${ }^{\mathrm{e}}$ siècle) comporte deux actes qui nous permettent de fixer une fourchette chronologique précise pour la mise en service du manuscrit. Ces deux actes, datés respectivement du 10 octobre 1360 et du 30 avril 1374 comportent, en suscription, la liste complète des chanoines de Saint-Materne, classés par ordre d'ancienneté (62). Parmi les chanoines cités dans la première liste et également commémorés dans lobituaire de Saint-Materne, aucun n'appartient à la couche initiale du document excepté Collard de Meeffe, cité le 7 décembre (63). Ce chanoine, qui est le plus récent parmi les personnages connus de la couche primitive, n'est plus mentionné dans la liste de 1374. Il est probablement décédé à cette date. Il n'est en effet jamais plus cité. Quant aux chanoines cités dans la seconde liste ainsi que dans l'obituaire, ils appartiennent toujours aux ajouts à la couche initiale. En conséquence, nous pouvons avancer que le scribe qui rédigea la première vague de commémorations opéra après le 10 octobre 1360, date à laquelle Collard de Meeffe vivait encore et où, dès lors, sa commémoration ne pouvait encore avoir été inscrite, et avant le 30 ávril 1374, date à laquelle il était décédé. Le terminus ante quem peut encore être avancé de deux ans. En effet, Jacques Bocheroul, commémoré le 27 octobre dans l'obituaire de Saint-Materne, dans le cadre d'un ajout à la couche primitive, est cité dans la liste de 1360 et est décédé avant 1372. Il est donc possible de ramener à 1372 au lieu de 1374, la date à partir de laquelle la main

(62) Cf. C.S.L., t. 4, pp. 328-329, 508.

(63) Cf. ob. Saint-Materne, $\mathrm{f}^{\circ} 49 \mathrm{r}^{\circ}$. 
initiale a cessé, au plus tard, d'opérer (64). Est-ce à dire, à présent, que c'est entre 1360 et 1372 que les chanoines de Saint-Materne commencèrent à célébrer la mémoire des défunts ? Certes non car, lorsque l'on examine les personnages les plus anciens de la couche première, on s'aperçoit que certains appartiennent au début du XIII ${ }^{e}$ siècle. Les défunts concernés par le fonds initial de l'obituaire sont situés chronologiquement entre ca 1205 (65) et 1360 . Il n'est évidemment pas envisageable que les commémorations aient été rédigées au jour le jour par la même personne durant toute cette période. Dès lors, nous pensons que l'obituaire de Saint-Materne n'est pas un manuscrit primitif mais un original récrit (66). Pour des raisons inhérentes au fait qu'il est un document vivant et perpétuel (illisibilité au fil des années, surcharge, détériorations du manuscrit), l'original primitif a été probablement abandonné et il fut décidé d'en rédiger un nouveau, duquel furent peutêtre exclues certaines notices issues de l'ancien obituaire et qui n'avaient plus aucune raison d'être. C'est cette opération de " recopiage " qui doit être située, nous semble-t-il, entre 1360 et 1372, au plus tard.

À la couche primitive ont été ajoutées de multiples notices. Disons en quelques mots. Ces commémorations ont été rédigées, dans des encres de couleurs variées, par de nombreuses mains, que l'on peut regrouper, selon l'écriture, en quatre groupes. Les quatre types d'écriture du bas moyen âge sont représentés : la littera textualis formata, la littera textualis tout court, la littera cursiva formata et la littera cursiva tout court (67). Sont concernés par ces ajouts: les personnages mentionnés dans les listes de 1360 et de 1374 et qui n'appartiennent pas à la couche primitive de l'obituaire, de très nombreux défunts de la fin

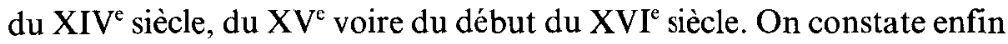
la présence de trois chanoines de Saint-Lambert décédés l'un en 1349 ,

(64) Cf. ob. Saint-Materne, $\mathrm{f}^{\circ} 42 \mathrm{r}^{\circ}$.

(65) Maître Élye, qui est peut-être commémoré le 15 avril (cf. ob. Saint-Materne, $\left.\mathrm{f}^{\circ} 18 \mathrm{r}^{\circ}\right)$, est décédé ap. 1205. De toute façon, l'évêque Hugues de Pierrepont, commémoré le 11 avril (ob. Saint-Materne, $\mathrm{f}^{\circ} 17 \mathrm{v}^{\circ}$ ) est décédé en 1229.

(66) L'original primitif est le document nécrologique initial de la communauté religieuse (cf. LEMAîTRE, Répertoire, p. 55). L'original récrit est la réfection de l'original primitif, non seulement copie mais également nouvelle mouture decelui-ci, tenant compte de corrections, de modifications, d'additions au texte initial (cf. LEMAÎTRE, Répertoire, pp. 46-49).

(67) Cf. Stiennon, Paléographie, pp. 119-120. À notre avis, distinguer de façon précise les multiples mains responsables des ajouts au fonds initial de l'obituaire n'a guẻre d'utilité. C'est la raison pour laquelle nous n'avons pas entrepris une telle tâche, qui n'est d'ailleurs pas préconisée systématiquement (cf. J.-L. LEMAîtTRE, Directives pour la préparation d'une édition de document nécrologique, BulLETIN PHILOLOGIQUe ET HISTORIQUE, 1979, p. 14). 
le second après 1358 et le dernier en 1366 (68). Il s'agit là, très probablement, de chanoines dont la commémoration a été inscrite longtemps après leur décès, a posteriori.

Venons-en, à présent, au problème du calendrier que comporte l'obituaire.

\section{QUELQUES MOTS A PROPOS DU CALENDRIER}

Notre propos ne sera pas de faire ici une analyse détaillée du calendrier. Nous laissons ce travail délicat aux spécialistes. Nous voudrions simplement émettre quelques observations à propos du sanctoral et des éléments astronomiques insérés dans notre calendrier.

\section{1) Le sanctoral}

Le calendrier (69), qui a été rédigé par la main initiale (70) et doit donc être daté des années 1360-1372 (71), est tronqué. En effet, plusieurs folios du manuscrit sont perdus (72). C'est la raison pour laquelle d'illustres saints du diocèse de Liège, tel saint Lambert, n'apparaissent pas. Toutefois, malgré ces défections, nous remarquons que de très nombreux saints, attachés d'une manière ou d'une autre à l'église de Liège, sont mentionnés. Citons, par exemple, saint Hadelin ( 3 février), saint Ursmer (18 avril), saint Gengulphe (11 mai), saint Servais (13 mai), saint Géréon (10 octobre), les onze mille Vierges (21 octobre), saint Hubert (3 novembre)... (73) À côté de ceux-ci, nous constatons la

(68) Il s'agit de Jean Haensank, de Gaufroid Rupin et de Pierre de Kirpech, commémorés respectivement le 13 février, les 5 et 12 mars dans l'ob. Saint-Materne, $\mathrm{f}^{\circ} 6 \mathrm{v}^{\circ}, 11 \mathrm{v}^{\circ}$ et $12 \mathrm{v}^{\circ}$.

(69) Il est nettement plus fourni que d'autres calendriers du diocèse de Liège tels l'ob. N.-D. Huy, éd. Deckers-Renardy, Bruxelles, 1975, ou l'obituaire de la collégiale SainteOde d'Amay, éd. Cl. MoREAu, mém. de lic. en Histoire, dactyl., Université de Liège, Liège, 1982-1983.

(70) Le sanctoral comporte deux ajouts postérieurs : la Visitation de la Vierge à sainte Élisabeth, le 2 juillet, et l'octave de la Visitation, le 9 juillet (ob. Saint-Materne, $\mathrm{f}^{\mathrm{o}} 31 \mathrm{r}^{\circ}, 32 \mathrm{r}^{\circ}$ ).

(71) À propos de la chronologie du document, cf. chapitre précédent, pp. 20-23.

(72) Nous renvoyons au chapitre 2,3, pp. 15-16.

(73) Sur les raisons de la présence de tous ces saints, cf. ob. Saint-Laurent (Liège), éd. CoENS, pp. 52-55. Sur ce document, dont la provenance est l'objet de controverses - il pourrait bien ne pas être issu de l'abbaye de Saint-Laurent de Liège mais être, en revanche, l'obituaire personnel de l'évêque de Liège, Henri de Verdun (1075-1091) -, cf. H. WelLMER, Persönliches Memento im deutschen Mittelalter, Stuttgart, 1973, pp. 109-113. - KuPPER, Liège et l'Église impériale, pp. 136 n. 142, 294 n. 26. - J. STIENNON, Note de paléogra- 
présence de saints assez éloignés géographiquement de Liège, tels sainte Brigide ou saint Kilien (le $1^{\text {cr }}$ février et le 8 juillet), ainsi que celle de martyrs cités en groupe ou collectivement. Nous mentionnerons Zoticus, Hereneius et Jacinctus (10 février), Vital, Félicole et Zénon (14 février), Alexandre, Eventius et Juvenal (3 mai), Basile, Kilien, Hyppolite, Denis, Géréon, Eustache, Saturnin et leurs compagnons respectifs ( 12 juin, 8 juillet, 13 août, 9 et 10 octobre, 2 et 29 novembre); les sept Dormants ( 27 juin), les sept frères martyrs (10 juillet), les Maures ( 15 octobre) et les quatre saints couronnés ( 8 novembre). Il convient de souligner que, par rapport au calendrier liégeois type (74), sont absents : sainte Appolonie ( 9 février), saint Albin (22 juin) et sainte Ode (27 novembre). Est par contre mentionné un saint non cité dans le sanctoral liégeois habituel et que nous n'avons d'ailleurs pas pu identifier : Marcius, le 14 février. Signalons, enfin, que le 27 mars et le 15 juillet sont fêtées respectivement la résurrection du Christ et la Division des apôtres. La première de ces fêtes, qui double la fête pascale, trouve son origine dans le raisonnement suivant : le Christ, homme parfait, ne peut avoir vécu que des années entières. Il est donc décédé le jour de sa conception, soit le 25 mars et est, par conséquent, ressuscité trois jours plus tard, le 27 mars. La Divisio apostolorum, quant à elle, commémore la séparation des douze apôtres, après la Pentecôte (75).

En ce qui concerne l'importance accordée aux diverses fêtes, nous avons remarqué que, par rapport au calendrier liégeois type (76), un plus grand nombre de fêtes sont rédigées à l'encre rouge. Les fêtes rubriquées supplémentaires sont les suivantes: Sainte-Agathe (5 février), Saint-Marcius (14 février), Saint-Mathieu (24 février), SaintGrégoire (12 mars), Sainte-Gertrude (17 mars), Saint-Benoît (21 mars), Saint-Ambroise (4 avril), Sainte-Marie l'Égyptienne (9 avril), SaintGeorges (23 avril), Saint-Domitien (7 mai), Saint-Barnabé (11 juin), Saint-Jean-Baptiste (24 juin) et son octave (1 $1^{\text {er }}$ juillet), l'octave de la Saints-Pierre-et-Paul (6 juillet) et de l'Assomption (22 août), SainteOde (24 octobre), la fête des âmes (2 novembre), Saint-Hubert (3 novembre), Sainte-Élisabeth (19 novembre), Sainte-Cécile

phie sur le calendrier-obituaire de Saint-Laurent de Liège (Brtixelles, B.R., 2031-32), dans Calames et cahiers. Mélanges de codicologie et de paléographie offerts à Jean Gilissen, Bruxelles, 1985, pp. 183-186.

(74) Publié dans Groterend, Zeitrechnung, t. 2, pp. 105-109.

(75) À propos de ces deux fêtes, cf. Platelle, Missel de Saint-Amand, pp. 141-142. C'est M. Ph. George qui nous a transmis cet article d'un accès difficile. Nous l'en remercions vivement.

(76) Cf. note 74 . 
(22 novembre), Saint-Trudon (24 novembre), la Conception de la Vierge ( 8 décembre) et Saint-Thomas ( 29 décembre). Pour des raisons évoquées plus haut, il n'est pas possible de calculer le nombre exact de fêtes par catégorie. Remarquons, néanmoins, que notre calendrier ne comporte pas de festa triplicia, ni semitriplicia. Nous constatons, en revanche, la présence de fêtes duplicia, de fêtes de IX et de III lectiones, de messes, de commémorations et de collectes (77). À propos de ces solennités, notre calendrier comporte quelques particularités, mineures, nous semble-t-il (78).

\section{2) Les éléments astronomiques du calendrier}

À côté des nombres d'or et des lettres dominicales, qui n'appellent aucun commentaire, il est une particularité de notre calendrier qui ne peut être passée sous silence. C'est le vers qui apparaît régulièrement en tête de chaque mois et qui est, de prime abord, sibyllin. Chacun de ces vers indique les deux jours qui, dans chaque mois, sont néfastes. $\mathrm{Ce}$ sont les jours appelés égyptiens ou égyptiaques, en latin dies egyptiaci, atri, mali ou egri (79). Ces jours sont réputés néfastes, en particulier pour toute émission de sang. Cette croyance, à laquelle on a attribué une origine égyptienne ( 80 ), est très ancienne. Ovide (43 A.C.N.-18 P.C.N.), déjà, dans les Fastes (I, 45-62), évoque les dies atri. À sa suite, ca 394, saint Augustin condamne ce qui n'est pour lui qu'une superstition (81) et c'est Bède le Vénérable qui en fera la théorie (82) en mentionnant d'abord trois puis soixante-trois jours particulièrement dange-

(77) Sur la classification des fêtes, dans le diocèse de Liège, cf. J. Daris, Classification des fêtes du diocèse de Liège, NOTICES HISTORIQUES SUR LES ÉGLISES DU DIOCìSE DE LIÈGE, t. 15, Liège, 1894, pp 29-30.

(78) Il y a parfois omission d'une qualité d'un saint (martyr...) ou du terme indiquant la solennité de la fête (duplex, semiduplex, missa). La fête liturgique est parfois suivie de collecta au lieu de commemoratio et inversement.

(79) À leur propos, cf. Grotefend, Zeitrechnung, t. 1, pp. 36-37. - Platelle, Missel de Saint-Amand, pp. 142-143. - Morel, Calendriers perpétuels, pp. 28-32. - Du Cange, art. Dies Aegyptiaci, Glossarium mediae et infimae latinitatis, t. 2, Paris, 1842, p. 846. - L. DE MAS LATRIE, Trésor de chronologie, d'histoire et de géographie pour l'étude et l'emploi des documents du moyen âge, Paris, 1889, col. 625.

(80) L'étude de l'origine de ces jours égyptiens nécessite assurément de plus larges recherches et de plus longs développements. Nous comptons revenir sur cet intéressant problème de chronologie dans une publication ultérieure.

(81) Les textes de saint Augustin et de Durand de Mende, dont il va être question plus bas, sont cités dans MOREL, Calendriers perpétuels, pp. 29-30.

(82) Bède LE VÉnÉrABle, De minutione sanguinis, sive de phlebotomia, dans J.-P. MIGNE, Patrologie latine, t. 90, 1862, col. 959-962. Sur Bède le Vénérable, cf. P. GodET, art. Bède le Vénérable. Dictionnaire de Théologie Catholique, t. 2, Paris, 1905, col 523-527. 
reux. À la fin du XIII ${ }^{\mathrm{e}}$ siècle, Guillaume Durand, évêque de Mende (83), ramène la croyance à une plus juste proportion en soulignant l'existence de seulement deux jours égyptiens par mois et en précisant la place qu'ils y occupent, par le biais d'une expression mnémotechnique (84). Comme nous l'avons dit plus haut, le vers qui inaugure chaque mois de notre calendrier précise la position des deux jours égyptiaques dans le mois. Chaque vers comporte, en effet, deux nombres. Le premier correspond au premier jour néfaste, en commençant par le début du mois ; le second correspond au second dies ater, en comptant, de manière inclusive, à partir de la fin du mois. Prenons l'exemple du mois de janvier. Le vers qui se trouve en tête de janvier contient les nombres un et sept. Les deux jours néfastes sont donc les $1^{\text {er }}$ et 25 janvier (31-7).

Il ne nous semble pas inutile de terminer ce chapitre par un essai de traduction de ces vers au sens assez contourné (85).

JANVIER : Iani prima dies et septima fine timetur.

Le premier jour de janvier et le septième avant la fin inspirent la terreur.

FÉVRIER: Ast Februi quarta est, precedit tertia finem.

En février, c'est le quatrième jour et le troisième avant la fin qu'il faut craindre.

Mars : Martius prima necat, cuius sub cuspide quarta est.

Le premier jour de mars tue ; le quatrième avant la fin nous place sous une pointe dangereuse.

AvRIL : Aprilis decimo est, undeno a fine minatur.

Le dixième jour d'avril et le onzième avant la fin nous menacent.

(83) Sur Guillaume Durand, évêque de Mende (ca 1230-ł1296), cf. L. FalLETT1, art. Guillaume Durand ou Durant, Dictionnaire de Droit Canonique, t. 5, Paris, 1953, col. 1014-1075.

(84) Cette phrase est la suivante: Augurior decios audito lumine clangor. Liquet olens abies coluit colus excute gallum. Chaque mot correspond à un mois du calendrier. La première lettre de chaque première syllabe correspond, suivant l'ordre qu'elle a dans l'alphabet, au premier jour néfaste du mois en commençant par son début ; la première lettre de chaque seconde syllabe correspond au second jour néfaste du mois, en comptant à partir de son terme. Cf. Grotefend, Zeitrechnung, t. 1, p. 36.

(85) La traduction des vers de janvier, février, mai, novembre et décembre, est celle présentée par Moré, Calendriers perpétuels, pp. 30-32. 
MAI : Tercius in maio lupus est et septimus anguis.

Le troisième jour de mai est un loup et le septième avant la fin est un serpent.

JUIN : Junius in decimo quindenum a fine salutat. Juin, en son dixième jour et le quinzième avant la fin vient nous saluer.

JUILLET : Tredecimus iulii decimo innuit ante kalendas.

Le 13 juillet et le dixième jour avant les kalendes sont un signe pour nous.

$[\ldots]$

SePtembre: Tertia septembris vulpes ferit a pede denam.

Le troisième jour de septembre est un renard et le dixième avant la fin frappe.

$[\ldots]$

NOVEMBRE : Quinta novembris acus (acris ?) vix, tertia mansit in urna. Le cinquième jour de novembre n'a guère de vigueur, le troisième jour avant la fin périt dans l'urne.

DÉCEMBRE: Dat duodena cohors septem inde decemque decembrer (decembris).

La douzième cohorte redoute le sept décembre et le dixième jour avant la fin.

\section{INTÉRETT DE L'OBITUAIRE DE SAINT-MATERNE}

Avant d'apprécier globalement la valeur et l'intérêt de notre document, il importe, nous semble-t-il, de préciser deux points essentiels : d'une part, la qualité des personnes dont le souvenir est célébré à SaintMaterne, d'autre part, la nature des fondations qui assurent ces commémorations.

Quels sont ceux qui fondent un service commémoratif à SaintMaterne ? Principalement des hommes (124 contre 11 femmes), des clercs, bien sûr (117 contre 18 laïcs) et, parmi ceux-ci, essentiellement des chanoines de Saint-Materne (67 sur 135 commémorations) (86). À

(86) Les chanoines relevant à la fois de Saint-Materne et d'un autre établissement ecclésiastique liégeois sont nombreux. L'interdiction de cumuler un canonicat à Saint-Materne avec un autre bénéfice ecclésiastique n'était donc pas respectée. 
côté de ceux-ci, on remarque la présence d'un évêque, Hugues de Pierrepont, d'un cardinal, Jean Gilles, de sept doyens (deux de Saint-Lambert, deux de la collégiale Saint-Denis, deux de Notre-Dame de Huy et un de la collégiale Sainte-Croix), de vingt-sept chanoines de Saint-Lambert et de quatre chanoines de la Petite-Table. À ces prébendiers vient s'ajouter un petit nombre d'ecclésiastiques (chapelains, vicaire, prêtre, diacre) et de laïcs (chevalier, bourgeois). Il convient de mettre l'accent sur le caractère familial de notre document. Assez nombreuses sont, en effet, les commémorations collectives (un fils et ses parents, deux frères, frère et sœur) (87) et celles, à des dates différentes, des membres d'une même famille (les Fize, un chanoine et ses parents) (88). Signalons encore que trois chanoines sont cités à plusieurs reprises, voire mensuellement, dans l'obituaire (89). Il convient enfin de mentionner la présence de quatre commémorations générales, le 30 janvier, le 9 mars, le 24 mai et le 2 novembre, jour des âmes (90).

En ce qui concerne les fondations, il est clair qu'elles sont constituées, pour la plupart, de mesures de grains, essentiellement de l'épeautre, beaucoup moins fréquemment du seigle. Ces céréales, tout comme les terres qui sont sporadiquement cédées à Saint-Materne, sont gagées sur des biens généralement situés en Hesbaye (91). Certaines donations sont composées de sommes d'argent. Les principales monnaies citées sont le sou, le denier, l'obole, le florin de Cologne et le marc. Quelques offrandes méritent encore d'être signalées. Sont en effet parfois cédés au chapitre de Saint-Materne : de la bière, une maison, en tout ou en partie, le produit d'une année de grâce, des chapons et des objets liturgiques (calices, pixide, aube et chasuble, corporal, custode, missel) (92). Après une étude du détail de notre document, il est temps, à présent, d'en souligner l'intérêt global.

(87) Cf., par exemple, le 23 avril, le 4 juin, le 31 octobre (ob. Saint-Materne, $\mathrm{f}^{\mathrm{o}} 19 \mathrm{v}^{\circ}$, $\left.26 \mathrm{v}^{\circ}, 42 \mathrm{v}^{\circ}\right)$.

(88) Ainsi les frères de Fize, les 11 janvier, 14 avril et 13 mai $\left(o b\right.$. Saint-Materne, $\mathrm{f}^{\circ} 4 \mathrm{v}^{\circ}$, $18 \mathrm{r}^{\circ}, 23 \mathrm{r}^{\circ}$ ) et, par exemple, Élie et sa mère, le 23 mars et le 15 avril, Henri Oneal et ses parents, le 30 janvier et le 3 novembre (ob. Saint-Materne, $\mathrm{f}^{\circ} 14 \mathrm{v}^{\circ}, 18 \mathrm{r}^{\circ} ; 5 \mathrm{v}^{\circ}, 43 \mathrm{r}^{\circ}$ ).

(89) Il s'agit d'Herman de Xanten, cité le premier de chaque mois (ob. Saint-Materne, $\left.\mathrm{f}^{\circ} 3 \mathrm{r}^{\circ}, 6 \mathrm{r}^{\mathrm{o}}, 11 \mathrm{r}^{\circ}, 16 \mathrm{r}^{\circ}, 21 \mathrm{r}^{\circ}, 26 \mathrm{r}^{\circ}, 31 \mathrm{r}^{\circ}, 38 \mathrm{r}^{\circ}, 43 \mathrm{r}^{\circ}, 48 \mathrm{r}^{\circ}\right)$, de Gauthier de Moorsele, le $1^{\text {er }}$ janvier, le $1^{\text {er }}$ mai et le $1^{\text {er }}$ septembre (ob. Saint-Materne, $\mathrm{f}^{\circ} 3 \mathrm{r}^{\circ}, 21 \mathrm{r}^{\circ}, 38 \mathrm{r}^{\circ}$ ), et de Henri d'Alken, le 23 juin et le 27 décembre ( $o b$. Saint-Materne, $\mathrm{f}^{\circ} 29 \mathrm{v}^{\circ}, 51 \mathrm{r}^{\circ}$ ).

(90) Ob. Saint-Materne, fo $5 \mathrm{v}^{\circ}, 12 \mathrm{r}^{\circ}, 24 \mathrm{v}^{\circ}, 43 \mathrm{r}^{\circ}$.

(91) Ceci n'a rien d'étonnant. Le sud de la Hesbaye est essentiellement consacré à la culture de l'épeautre. Cf. L. ZYLBERGELD, Le prix des céréales et du pain à Liège dans la première moitié du XIII siècle, R.B.P.H., t. 51, 1973, pp. 287-288 et carte, p. 277.

(92) Cf. les 15 avril et 22 août (ob. Saint-Materne, $\left.\mathrm{f}^{\circ} 18 \mathrm{r}^{\circ}, 36 \mathrm{v}^{\circ}\right)$. 
Il en va des sources nécrologiques comme des reliques. Les négliger, c'est s'exposer à ne rien comprendre à la société médiévale (93). Le formidable apport de cette documentation, à tous les domaines de la recherche historique, est sans conteste (94). Il est possible, croyonsnous, d'étayer cette opinion grâce à l'obituaire de Saint-Materne. Il apporte, en effet, nombre d'indications à des disciplines aussi diverses que la prosopographie, l'onomastique, l'histoire liturgique, sociale et économique.

Évoquons tout d'abord la prosopographie et l'histoire sociale. Le chapitre de Saint-Materne et son organisation sont mal connus, surtout pour les deux premiers siècles de leur histoire. Des chanoines de cette époque, nous en ignorons parfois jusqu'à l'identité. Or, l'obituaire peut pallier, partiellement du moins, ces lacunes. On y trouve, en effet, mentionnés de très nombreux chanoines de Saint-Materne. À partir de ces noms, il nous a souvent été possible, suite à des recherches approfondies, de retracer leur biographie ou, à tout le moins, de préciser leur chronologie. Dès lors, nous pensons ne pas pécher par orgueil lorsque nous affirmons que les éléments biographiques qui forment l'essentiel de l'annotation à notre édition, constituent, avec celle-ci, une contribution appréciable à l'histoire de la vie religieuse liégeoise (95). S'il donne une liste de noms, l'obituaire permet également, très souvent, de préci-

(93) Cette phrase percutante mais pleine de sagesse est due à la plume de notre maitre A. Joris dans sa préface à Ph. George, Les reliques de Stavelot-Malmedy, Malmedy, 1989, p. 5.

(94) Cf. Huyghebaert, Documents nécrologiques, pp. 63-70. - J.-L. Lemaître, Les obituaires français. Perspectives nouvelles. Revue d'HistoIre de l'ÉGLise de France, t. 64, 1978, p. 71. - ID., Les obituaires des chapitres cathédraux du Languedoc, dans Le monde des chanoines (XI'-XIV s.), Cahiers de Fanjeaux, t. 24, 1989, pp. 135-146.

(95) LAHAYE, Saint-Materne, pp. 111-150, dresse une nomenclature des chanoines de Saint-Materne. Malheureusement, celle-ci est consacrée volontairement et exclusivement aux chanoines ayant vécu à partir du XV siècle. L. Lahaye passe donc sous silence les chanoines des $\mathrm{XIII}^{\mathrm{e}}$ et $\mathrm{XIV}^{\mathrm{e}}$ siècles. Dans la biographie des chanoines du XV siècle, on regrettera qu'il ne fasse pratiquement pas usage des éditions de sources, liégeoises ou autres, comme le C.S.L. Il est également pour le moins étonnant qu'il ne tire pas parti de ses propres travaux tels LaHAYE, Saint-Jean, 2 vol., Bruxelles, 1921-1931. Par ailleurs, et sans vouloir ici ternir la réputation d'un érudit qui a beaucoup apporté à l'historiographie liégeoise, nous devons bien constater une certaine imprécision dans la mention de ses sources. Les seules indications que $\mathrm{L}$. Lahaye donne à propos des chanoines des $\mathrm{XIII}^{\mathrm{e}}$ et $\mathrm{XIV}^{\mathrm{e}}$ siècles sont puisées dans ce qu'il appelle des nomenclatures. Celles-ci se révèlent être, essentiellement, des suscriptions d'actes tirés du cartulaire de Saint-Materne et publiés dans C.S.L., t. 3 , p. 423 ; t. 4, pp. $328-329,508$. Ces actes, dont il ne mentionne pas la provenance avec précision, sont datés du jour, du mois et de l'année et pas seulement de l'année comme risque de le faire croire l'article de LAHAYE, Saint-Materne, pp. 109-110. 
ser la date de mort des personnages qui y sont mentionnés. L'obituaire de Saint-Materne ne faillit pas à la règle : il nous a permis de fixer avec précision le jour du décès de plusieurs chanoines (96).

À côté des anthroponymes, l'obituaire comporte également de multiples noms de lieux. La forme de ces toponymes est très souvent originale et il est parfois possible d'en étudier l'évolution lorsque ces formes sont citées dans des notices réparties sur quatre siècles (XIII ${ }^{\mathrm{e}}-\mathrm{XVI}^{\mathrm{e}}$ siècles). Il est clair que l'onomastique ne peut faire fi de la mine de renseignements que constitue, à l'évidence, l'obituaire de Saint-Materne.

L'histoire liturgique, elle aussi, tirera profit de notre document, ne fût-ce que du calendrier qu'il comporte. Il convient en effet de rappeler que l'obituaire de Saint-Materne est l'un des rares documents nécrologiques liégeois à présenter un calendrier liturgique complet. Nous nous sommes efforcé d'en montrer tout l'intérêt dans le chapitre précédent. Nul doute que les spécialistes trouveront bien d'autres raisons de s'y arrêter. À côté du calendrier, l'obituaire de Saint-Materne recèle également plusieurs commémorations très circonstanciées où est explicitée, non seulement la fondation du défunt, mais aussi les modalités d'utilisation de cette donation, des indications qui ne peuvent qu'intéresser les spécialistes de la liturgie.

Il est enfin un domaine qui ne peut ignorer les documents nécrologiques, c'est l'histoire économique. On peut prétendre qu'elle doit être écrite à partir de séries chiffrées, complètes et continues, il n'empêche que des documents où sont précisés le nom du donateur d'une terre ou d'une quantité précise de grains, la provenance de l'un et de l'autre ou son affectation ne sont pas légion au moyen âge, particulièrement dans nos régions (97). Or, les sources nécrologiques apportent souvent ce type de précisions, bien entendu pour les seules fondations obituaires. Nous croyons toutefois ne pas nous tromper lorsque nous affirmons que notre document sera un complément essentiel au cartulaire de Saint-Materne pour tout chercheur qui, à l'avenir, voudrait

(96) Par exemple, celui de Gilles de Sainte-Marie, chanoine de Saint-Materne, commémoré le 8 février (ob. Saint-Materne, $\mathrm{f}^{\circ} 7 \mathrm{r}^{\circ}$ ) ou celle d'Antoine de Fize, chanoine de SaintLambert, commémoré le 14 avril (ob. Saint-Materne, $\mathrm{f}^{\circ} 18 \mathrm{v}^{\circ}$ ). Sur l'emploi des obituaires dans la résolution d'un problème de chronologie, cf. la démonstration magistrale de notre maître, J.-L. KUPPER, dans Les "Gesta pontificum Leodicensis Aecclesiae " du chanoine Anselme, Problématique de l'Histolre liéGeoise, Liège, 1981, pp. 30-31.

(97) Le premier polyptyque liégeois date du début du XIV siècle. Cf. D. VAN DERVEEGHDE, Le polyptyque de 1280 du chapitre de la cathédrale Saint-Lambert à Liège, Bruxelles, 1958 et A. JorIs, C.R., R.B.P.H., t. 37, 1959, pp. 813-817. 
étudier le domaine et les sources de revenus de cet établissement religieux (98).

Nous pensons avoir ainsi fait le tour des principaux domaines historiques auxquels l'obituaire de Saint-Materne apporte son lot d'informations. Nous espérons, par ailleurs, avoir plaidé avec éloquence pour des sources qui, pour la plupart, attendent encore leur éditeur.

\section{PRÉSENTATION DE L'ÉDITION}

Nous aimerions apporter quelques précisions quant à l'édition que nous présentons dans cet article. Nous avons bien entendu transcrit, le plus fidèlement possible, le texte original, seul document à notre disposition. Le seul changement systématique que nous ayons consenti est la transcription des nombres (quantités dans les fondations), sous une seule et unique forme : les chiffres romains (le texte original comporte tantôt des lettres, tantôt des chiffres romains). Nous nous sommes efforcé de respecter scrupuleusement les règles d'édition édictées par la C.R.H. Le texte est composé en deux types de caractères : les petites capitales correspondent au fonds initial de l'obituaire, les italiques, aux multiples ajouts faits à la couche primitive. Le sigle [...] indique la perte d'un ou de plusieurs folios du manuscrit. Chaque notice italique fait l'objet d'une note dans l'apparat critique. Nous y mentionnons le type d'écriture à laquelle se rattache la main qui rédigea l'ajout en question et l'époque de la rédaction (celle-ci peut être précisée, éventuellement, dans l'annotation, d'après la date de décès des personnages cités). Les écritures ont été groupées en quatre groupes, désignés par les lettres $B$, $C, D$ et $E$, la lettre $A$ étant réservée à la main qui rédigea la couche primitive du document. $B$ correspond aux notices rédigées en littera textualis formata, $C$, à celles rédigées en littera textualis, $D$, à celles rédigées en littera cursiva formata et enfin $E$, à celles rédigées en littera cursiva. Sont également mentionnées dans l'apparat critique les suscriptions au texte initial et les particularités physiques (détériorations, taches...) et textuelles (notula...) du document.

Dans l'annotation, nous avons tenté d'identifier tout ce qui, dans le texte, est susceptible d'interpeller le lecteur : personnages, lieux, fêtes, pour l'essentiel.

(98) On trouvera le témoignage éclatant de l'inestimable apport d'un obituaire à la recherche historique dans l'introduction de notre édition de l'O.S.L., Bruxelles, 1991 (sous presse). 
Les personnages ont été identifiés en assez grand nombre ou, à tout le moins, voient leur chronologie précisée, soit grâce à nos recherches, soit, éventuellement, par leur place dans la notice du jour.

Les formes des toponymes ont été élucidées grâce aux ouvrages suivants: C.S.L. - J. Herbillon, Toponymes hesbignons, B.T.D., t. 28-52, 1954-1978. - Liste alphabétique des communes du royaume de Belgique, Bruxelles, 1978.

Il nous a enfin semblé utile d'apporter quelques précisions à propos des saints mentionnés dans le calendrier : nom, si possible, en français, qualités, jour de la fête liturgique. Nous n'avons pas jugé bon de mentionner l'année de décès de chaque saint. Celle-ci est rarement connue avec précision. Elle tient souvent davantage de la légende que de l'histoire. Outre dans les autres calendriers-obituaires du diocèse de Liège, mentionnés dans la bibliographie, les informations à propos du sanctoral ont été puisées, pour l'essentiel, dans les ouvrages suivants: A. GIRY, Manuel de diplomatique, Paris, 1894. - - H. Grotefend, Taschenbuch der Zeitrechnung des deutschen Mittelalters und der Neuzeit, $11^{\mathrm{e}}$ éd., sous la dir. de Th. ULRICH, Hanovre, 1971. - ID., Zeitrechnung des deutschen Mittelalters und der Neuzeit, 2 vol., Hanovre, 1891-1892.

Quelques mots, à présent, sur l'apparat bibliographique de l'annotation. Les ouvrages cités à plusieurs reprises sont désignés de façon abrégée. On trouvera la résolution de ces abréviations dans la bibliographie, au début de cet article. Enfin, certains termes, cités fréquemment, ont également été tronqués (99).

(99) En voici la liste : ap. : après ; arr. : arrondissement; art. : article ; av. : avant ; ch.-1. : chef-lieu ; comm. : commune ; conf. : confesseur ; dép. : département ; dir. : direction; éd. : édition ; pr. : province ; repr. : reproduction ; sér. : série ; s. : siècle ; † : décédé. 


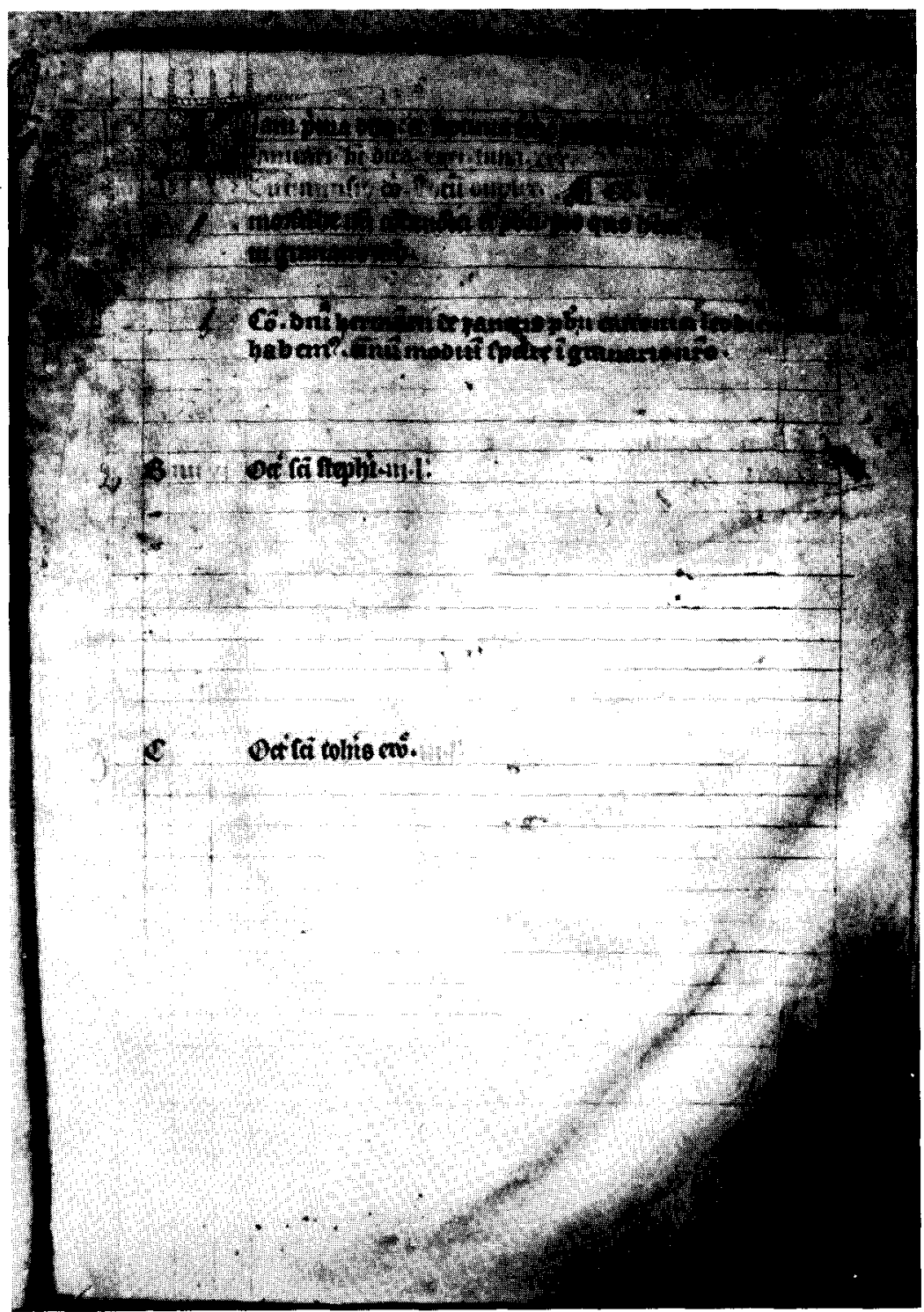

Ob. Saint-Materne, $\mathrm{f}^{\circ} 3 \mathrm{r}^{\circ}$. 



\section{ÉDITION DE L'OBITUAIRE}

\section{JANUARIUS HABET DIES XXXI, LUNA XXX}

III A kalendas

(1 janvier)

Circumcisio Domini (2). Totum duplex.

Commemoratio magistri Walteri de Morselhe (3), nostri concanonici et presbyteri, pro quo habemus III modios spelte in granario nostro.

Commemoratio domini Hermanni de Zanctis (4), presby-

(1) Sur les dies (a)egyptiaci, cf. introduction, pp. 25-27. La forme habituelle est : Jani prima dies et septima a fine timetur. Cf. Grotefend, Zeitrechnung, t. 1, p. 36.

(2) Circoncision. Fête : le $1^{\text {er }}$ janvier.

(3) Maître Gauthier de Moorsele (pr. Flandre occidentale, arr. Courtrai, comm. Wevelgem), chanoine de Saint-Materne, décédé depuis peu le 8 février 1322. Cf. RENARDY, Les maitres universitaires, p. 231. - C.S.L., t. 3, p. 234.

(4) Herman de Xanten (R.F.A.; pr. Rhénanie du Nord-Westphalie; cercle de Moers), chanoine et receveur du chapitre de la cathédrale de Liège à partir du 18 mars 1339 , prévôt de la collégiale Saint-Pierre le 7 janvier 1350, charge qu'il résigna ca 1364 , vice-doyen de Saint-Lambert en 1356, doyen de la collégiale de Huy de 1363 à 1379 et abbé séculier de Visé dès 1364. Il mourut le 12 septembre 1382. Cf. Chot-Stassart, p. 223. - GÉNICOT, Les chanoines de Huy, p. 68. - C.S.L., t. 3, pp. 560, 568, 571, 601, 611,614 ; t. 4, pp. $65,123,149,153,157,160,167-169,179,188,196,197,212,225,276$, $287,294,307,308,314,338,342,364,366,375,402,413,425,440,447,464,484,500$, $537,538,585,591,602,615$; t. 6 , pp. $91,122,126,128,140-141,146,407$. - BerLiÈre, Suppliques Clément VI, $\mathrm{n}^{\mathrm{os}} 1516,1816$, pp. 408-409, 473. - BERLIÈRE, Instrumenta Miscellanea, $\mathrm{n}^{\circ}$ 53, p. 36. - PAQUay, Cartulaire N.-D. Tongres, t. 1, pp. 420, 466, 467-468. - Poncelet, Sainte-Croix, t. 1, pp. 270, 280, 281. - Cuvelier, Val-Benoit, pp. 559, 667. - Poncelet, Saint-Pierre, pp. XXIV, 73. - Poncelet, F.E.M., pp. 306, 646. - VAN Der MADE, Guillemins, pp. 51-55, 60-61, 68, 76, 77-79, 81-82. Herman de Xanten a, semble-t-il, rédigé plusicurs testaments. On en connaît au moins deux : l'un, daté du 
teri canonici Leodiensis, pro quo habemus I modium spelte in granario nostro $\left({ }^{a}\right)$.

\section{B IIII nonis}

(2 janvier)

Octava Sancti Stephani (5). III lectiones.

XI C III nonis

(3 janvier)

Octava Sancti Johannis evangeliste (6). III lectiones.

Octava Sanctorum Innocentium (7). III lectiones.

XIX E nonis

(5 janvier)

Vigilia.

VIII F VIII idus

(6 janvier)

Epyphania Domini. Totum duplex.

$\left[4 r^{\circ}\right]$

G VII idus

( 7 janvier)

Commemoratio Lamberti de Op Here (8), canonici

$\left(^{a}\right) B$ : écriture de type littera textualis formata $\left(X I V^{e}-X V^{c}\right.$ s.).

24 juin 1371 (cf. VAN DER MADE, Guillemins, pp. 60-61); l'autre, du 27 septembre 1379 (cf. C.S.L., t. 6, pp. 140-141. - J. STEKKE, Contrats de mariage et testaments hutois (1270-1795), A.C.H.S.B.A., t. 26, 1956, p. 90). Herman de Xanten est commémoré le 19 septembre dans l'ob. N.-D. Huy, éd. Deckers-Renardy, pp. 190-191. Pour son épitaphe à la cathédrale Saint-Lambert, cf. Naveau-Poullet, t. 1, p. 7.

(5) Saint Êtienne, protomartyr. Fête : le 26 décembre.

(6) Saint Jean l'Évangéliste. Fête : le 27 décembre.

(7) Saints Innocents, martyrs. Fête : le 28 décembre.

(8) Lambert de Opheers est cité comme chanoine de Saint-Materne en 1290 et en 1302 ; comme chanoine de la collégiale Saint-Jean l'Évangéliste, à Liège, en 1311. Cf. Hemricour T, t. 1, p. 343 ; t. 2, p. 225. - PonCelet, Sainte.Croix, t. 1, p. 68. - C.S.L., t. 3, p. 12. - Vannérus, Documents, pp. 222, 230. - Bormans, Saint-Denis, p. 97 . LAHAYE, Saint-Jean, t. 1, p. XCII. 
Sancti Johannis, pro quo habemus $\mathrm{X}$ solidos bone et XXX quartas cervisie recipiendas sive recipiendos supra quasdam domos quae vocantur sor le Bresine Bastin iuxta stalla ante Sanctum Servatium (9), de quibus reddimus II denarios bone celerario Sancti Petri $\left({ }^{a}\right)$.

XVI A VI idus

(8 janvier)

V

B V idus

$(9$ janvier)

$\left[4 v^{\circ}\right]$

C IIII idus

(10 janvier)

XIII D III idus

(11 janvier)

Commemoratio domini Egidii de Fies (10) pro quo habemus III modios spelte $\left(^{b}\right)$.

$\left(^{a}\right)$ En bas de page et sur le bord extérieur $d u f^{\circ} 4 r^{\circ}$, notula (indications comptables datant de l'époque moderne). - $\left(^{b}\right) B$ : écriture de type littera textualis formata $\left(X I V^{e}-X V^{e} s\right.$.).

(9) Sur Saint-Servais, paroisse et église de Liège, cf. Gobert, Les rues de Liège, t. 5, pp. 311-323. - Lahaye, Paroisses, p. 14 et passim.

(10) Selon LaHAYe, Saint-Materne, p. 135, Gilles de Fize, chanoine de Saint-Materne, est décédé le 11 janvier 1381 . Le 20 juin 1381, certaines dispositions successorales sont prises par ses exécuteurs testamentaires (cf. C.S.L., t. 4, p. 598). Il est encore cité comme chanoine de Saint-Materne en 1368 et en 1374 (cf. C.S.L., t. 4, pp. 468, 508). Le chanoine commémoré ce 11 janvier est, semble-t-il, apparenté à deux autres chanoines du même nom, Antoine et Jean de Fize, cités respectivement le 14 avril et le 13 mai dans l'ob. Saint-Materne, $\mathrm{f}^{\circ} 18 \mathrm{r}^{\circ}, 23 \mathrm{r}^{\circ}$ - en 1376, Giles de Fize est exécuteur testamentaire d'Antoine de Fize (cf. C.S.L., t. 6, p. 134). Cf. également BerLiÈre, Suppliques Innocent VI, $\mathrm{n}^{\circ} 1343$, p. $548 \mathrm{n}$. 2. Il n'est pourtant pas le frère de ces deux chanoines, appelé, Jui aussi, Gilles de Fize (cf. C.S.L., t. 6, p. 134), chapelain de Saint-Lambert dès 1344 (cf. C.S.L., t. 4, p. 22) et chanoine de la collégiale Saint-Denis de Liège en 1364 (cf. C.S.L., t. 4, p. 374). Cf. également Hemricourt, t. 1, p. 448 ; t. 2, p. 257 . Il convient de remarquer qu'un Gilles de Fize est cité, notamment comme chapelain de la Cathédrale, dès 1315 (cf. C.S.L., t. 3, pp. 156, 332, 508, 583, 584 ; t. 6, p. 54. - SCHOONBROODT, Val-Saint-Lambert, t. 1, pp. 183, 195). Un autre, chantre de Notre-Dame de Tongres, est cité jusqu'en 1399 (cf. PaQuay, Cartulaire N.-D. Tongres, t. 1, pp. 483, 521, 528, 541, 604, 609, 611, 612. - PonceleT, Sainte-Croix, t. 1, p. 285). 
II $\quad$ E II idus

(12 janvier)

Commemoratio domini Egidii de Roussetes (11), sacerdotis canonici Sancti Materni, pro cujus anniversario faciendo habemus III modios spelte cum dimidio recipiendos super I bonuarium terre apud Wamon (12).

$\left[5 r^{\circ}\right] \quad$ IX D VIII kalendas

(25 janvier)

Conversio Sancti Pauli (13). IX lectiones. Proiecti (14) martyris. Collecta.

E VII kalendas

(26 janvier)

Policarpi (15) episcopi et martyris. Commemoratio.

XVII F VI kalendas

(27 janvier)

Johannis (16) episcopi et confessoris. Commemoratio.

$\left[5 v^{\circ}\right]$ VI G V kalendas

(28 janvier)

Octava Sancte Agnetis (17) virginis. III lectiones.

(11) Gilles dit de Ros(s)ettes est cité comme chanoine de Saint-Martin le $1^{\text {er }}$ juillet 1302 (cf. VanNÉrus, Documents, p. 222). Il y a probablement eu confusion entre Saint-Martin et Saint-Materne. Gilles de Rosettes est par ailleurs commémoré le 11 janvier dans l'O.S.L., éd. MARCHANDISSE, p. 9 et n. 41.

(12) Wamont/Waasmont, commune de Houtain-l'Évêque/Walshoutem (pr. Brabant, arr. Louvain, comm. Landen).

(13) Conversion de Saint-Paul. Fête : le 25 janvier.

(14) Saint Prix, Pregt, Prey, Priest, diacre, martyr. Fête : le 25 janvier.

(15) Saint Polycarpe, évêque et martyr. Fête : le 26 janvier.

(16) Saint Jean Chrysostome. Fête : le 27 janvier.

(17) Sainte Agnès, vierge et martyre. Fête: le 21 janvier. Octave (festum Agnetis secundo) : le 28 janvier. 
A IIII kalendas

(29 janvier)

Valerii (18) episcopi et confessoris. Commemoratio.

XIIII B III kalendas

(30 janvier)

Aldegundis (19) virginis. Collecta.

Commemoratio fratrum nostrorum canonicorum Sancti Materni defunctorum pro quorum memoria habemus II modios spelte recipiendos super II bonuariis terre jacentibus in territorio de Vorrus (20), legatis per $\mathrm{Ar}<$ nuldum $>$ de Okires (21).

Eodem die commemoratio domini Gerardi de Oxhen (22), canonici Leodiensis presbyteri, pro quo habemus XII sextarios spelte apud Puchey (23).

Eodem die commemoratio Henrici Oneal, patris domini

(18) Saint Valerius, évêque, conf. Fête : le 29 janvier.

(19) Sainte Aldegonde, abbesse. Fête : le 30 janvier.

(20) Il s'agit soit de Voroux-lez-Liers (pr. Liège, arr. Liège, comm. Juprelle), soit de Voroux-Goreux (pr. Liège, arr. Waremme, comm. Fexhe-le-Haut-Clocher).

(21) Il s'agit peut-être d'Arnoul de Cokiers, cité comme chanoine de Saint-Materne, le 12 janvier 1302. Cf. C.S.L., t. 3, p. 12.

(22) Maître Gérard Chabot, dit d'Ochain, doyen de la collégiale Saint-Denis de Liège dès le 23 août 1305, chanoine prébendé de Saint-Paul de Nivelles avant le 21 avril 1319, date à laquelle il reçoit une expectative de prébende à Saint-Lambert ; prévót de la collégiale Saint-Paul de Liège (1323) et abbé de Ciney (dès le 20 juillet 1332). Il meurt le 30 janvier 1348. Cf. ReNardy, Les maitres universitaires, p. 239. - ChOt-Stassart, p. 138. - G. Despy, Les dignitaires du chapitre de Ciney du $X I I^{e}$ au $X V^{e}$ siècle, NAMURCUM, t. 26, 1952, p. 45. Divers documents relatifs à ses dispositions testamentaires sont contenus dans C.S.L., t. 4, pp. 85-86, 96-103, 134-136; t. 6, pp. 99, 100. Pour son épitaphe à Saint-Lambert, cf. Naveau-Poullet, t. 1, p. 48. Il est commémoré le 30 janvier dans l'O.S.L., éd. MarCHANDisse, p. 16 et n. 76 et dans l'ob. Neufmoustier, éd. Closset, t. 1, p. 5 .

(23) Il s'agit soit de Pousset (pr. Liège, arr. Waremme, comm. Remicourt), soit de Poucet (pr. Liège, arr. Waremme, comm. Hannut). 
Henrici Oneal (24), concanonici nostri, pro quo habemus VI sextaria spelte apud Villari Episcopi $\left(^{a}\right)(25)$.

III C II kalendas

(31 janvier)

NOX HABET HORAS XVI, DIES VIII

\section{FEBRUARIUS HABET DIES XXVIII, LUNA XXIX}

D kalendas

(1 février)

Ignatii (26) episcopi $\left({ }^{b}\right)$. III lectiones. Brigide (27) virginis. Collecta.

Commemoratio domini Hermanni de Zanctis (28), presbyteri canonici Leodiensis, pro quo habemus I modium spelte in granario nostro $\left({ }^{c}\right)$.

XI E IIII nonis

(2 février)

Purificatio Beate Marie virginis (29). Totum duplex.

$\left({ }^{a}\right)$ B : écriture de type littera textualis formata $\left(X I V^{r e}-X V^{p} s.\right)$. $-\left({ }^{b}\right)$ et martyris, suscrit. - ( $\left.{ }^{c}\right)$ D : écriture de type littera cursiva formata $\left(X I V^{e}-X V^{e}\right.$ s.).

(24) Henri Oneal ou Onelli, chanoine de Saint-Materne, est cité du 30 octobre 1367 au 30 juin 1386. Cf. LAHAYE, Saint-Materne, p. 110. C.S.L., t. 4, pp. 450, 506, 508-510, $598,621,623,624,645$.

(25) Villers-l'Évêque (pr. Liège, arr. Liège, comm. Awans).

(26) Saint Ignace, évêque, martyr. Fête : le $1^{\text {er }}$ février.

(27) Sainte Brigide, vierge et martyre, abbesse fondatrice du monastère de Kildare

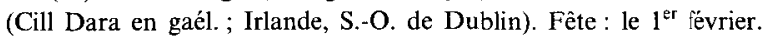

(28) Cf. note 4.

(29) Purification de la Vierge. Fête : le 2 février. 
XIX F III nonis

(3 février)

Hadelini (30) confessoris. III lectiones. Blasii $\left(^{(}\right)(31)$ martyris. Collecta. Waldetrudis (32) virginis. Commemoratio.

$\left[6 v^{\circ}\right]$ VIII G II nonis

(4 février)

A nonis

(5 février)

Agathe (33) virginis et martyris. IX lectiones $\left({ }^{b}\right)$.

Commemoratio magistri Egidii Gilorini(34), dyaconi canonici nostri, pro quo habemus II modios spelte apud Villari Episcopi (35) acquisitos a Johanne delle Neffe (36). Item VII sextarios spelte de $X X$ sextariis emptis a Johanne delle Soxhe de Fexhe $\left.{ }^{c}\right)$ (37).

$\left(^{(}\right)$episcopi, suscrit. - $\left({ }^{b}\right)$ duplex, suscrit. - ( $\left.{ }^{c}\right)$ B: écriture de type littera textualis formata $\left(X I V^{e}-X V^{e}\right.$ s. $)$.

(30) Saint (H)Adelin, conf., abbé de Celles. Après la translation de ses reliques, en 1338, il devint le patron de Visé (cf. BerLikère et autres, Monasticon belge, t. 1, Liège, s.d., p. 56 (reproduction anastatique de l'éd. 1890). Fête : le 3 février.

(31) Saint Blaise, évêque, martyr. Fête : le 3 février.

(32) Sainte Waudru, vierge, abbesse. Fête : le 3 février, à Liège.

(33) Sainte Agathe, vierge et martyre. Fête : le 5 février.

(34) Maître Gilles Gilorin(i) ou Gilloriro, chanoine de Saint-Materne, est cité le 22 novembre 1343 comme notaire, le 20 avril 1345 comme clerc du diocèse de Liège, le 9 mars 1357 et le $1^{\text {er }}$ octobre 1360 comme chanoine de Saint-Materne. Il est décédé avant le 30 avril 1374. Cf. C.S.L., t. 4, pp. 21, 255, 328, 508. - BERL.ièRE, Lettres Clément VI, $\mathrm{n}^{\circ}$ 1536, p. 564. - LAHAYE, Saint-Materne, p. 109. - RENARDY, Les maîtres universitaires, ne le mentionne pas.

(35) Villers-l'Évêque. Cf. note 25.

(36) Le 5 mai 1354, l'abbesse du Val-Benoît (abbaye bénédictine de Liège) concède l'exploitation d'une houillère, entre autres, à Jean delle Neffe (cf. CuveliEr, Val-Benoît, pp. 471-473). Un citain de Liège du même nom fait divers reliefs en 1351,1357 et 1361 (cf. PonCElet, F.E.M., pp. 392, 578, 720).

(37) En 1306, Jean delle Soxhe (lieu-dit à Fexhe-Slins, pr. Liège, arr. Liège, comm. Juprelle) de Fexhe était marié à Maroie de Mont, selon Hemricourt, t. 1, p. 330; t. 2, p. 373. - CuVelier, Val-Benoît, p. 310. Est-ce le même personnage qui, sous le nom de Jean de Gembluz, dit del Sohe de Fehe, est cité le 14 décembre 1363 comme ayant partie liée avec le chapitre de Saint-Materne (cf. C.S.L., t. 4, p. 393)? D'autres personnages portent également le nom de Jean de Fexhe : l'un fruitier, cité en 1334 et en 1345, décédé (?) en 1359 ; l'autre, bourgeois de Tongres, cité en 1351 et en 1361. Cf. PonCeleT, F.A.M., p. 345 . - PONCELET, F.E.M., pp. 135, 141, 399, 640, 720. 
XVI B VIII idus

(6 février)

Amandi (38) et Vedasti (39) episcoporum et confessorum. Collecta.

$\left[7 r^{\circ}\right]$ V C VII idus

(7 février)

D VI idus

(8 février)

Commemoratio Eve, mulieris, pro qua habemus $\mathrm{V}$ modios spelte et II sextarios super terram jacentem apud Villare (40) quae quondam fuit magistri Helye (41).

Eodem die commemoratio domini Egidii de Sancta Maria (42), canonici Sancti Materni et sacerdotis, pro quo habemus XII sextarios spelte supra granarium nostrum.

(38) Saint Amand, évêque, conf. Fête : le 6 février.

(39) Saint Vaast, évêque, conf. Fête : le 6 février.

(40) Villers-l'Évêque. Cf. note 25.

(41) RENARDY, Les maîtres universitaires, p. 210, mentionne un maître Élie, cité de 1212 au 24 mai 1224. Celui-ci est commémoré le 3 décembre dans das älteste Aachener Totenbuch, éd. E. Teichmann, Zeitschrift des Aachener Geschichtsvereins, t. 38 , 1916, p. 144. Or, le magister ici cité est probablement celui qui est commémore le 15 avril dans l'ob. Saint-Materne, $\mathrm{f}^{\circ} 18 \mathrm{r}^{\circ}$. Les dates de commémoration ne coïncident donc pas. Toutefois, un maître Élie est mentionné, le 14 avril, dans le Polyptyque de Saint-Lambert, p. 186, et est identifié comme étant un chanoine du XII ${ }^{\mathrm{e}}$ siècle. IL s'agit peut-être de Élie, Hélie ou Hélyas de Bouillon, chanoine de Liège de 1178 à 1205, écolâtre de Tirlemont (pr. Brabant, arr. Louvain, comm. Tirlemont), compagnon d'exil à Reims de l'évêque de Liège Albert de Louvain. Cf. à son propos, ChOT-STASSART, p. 28. - KuPPER, Liège et l'Église impériale, p. 181 n. 392. Maître Élie est commémoré le 14 avril dans l'O.S.L., éd. MARChANDISSE, p. 51 et n. 304.

(42) Gilles de Sainte-Marie ou de Notre-Dame, chanoine de Saint-Materne, est cité du 24 décembre 1318 au 12 mars 1349. Il était déjà mort le 25 janvier 1352. Il est donc probablement décédé aux alentours du 8 février, en 1350 ou en 1351 . Il fut, notamment, exécuteur testamentaire de Lambert de Stavelot, commémoré le 16 juin dans l'ob. Saint-Materne, f $28 \mathrm{v}^{\circ}$. Cf. C.S.L., t. 3, pp. 187 et n., 189, 208, 234, 320, 393, 423; t. 4, pp. $34,63-64,148,162$; t. 6 , p. 73 . - Poncelet, Sainte-Croix, t. 1, pp. 155-156. LAHAYE, Saint-Materne, p. 109. 
XIII E V idus

( 9 février)

Commemoratio Johannis de Hers (43), subdiaconi, pro quo habemus in granario nostro I modium siliginis.

$\left[7 v^{\circ}\right] \quad$ II $\quad$ F $\quad$ IIII idus

(10 février)

Zotici, Herenei et Jacincti (44) martyrum. Collecta. Scolastice (45) virginis. Collecta. Sotheris (46) virginis. Collecta.

G III idus

(11 février)

$\mathrm{X}$

A II idus

(12 février)

Commemoratio domini Andree de Insula (47), concanonici nostri et presbyteri, pro quo habemus XIX sextarios spelte.

Commemoratio domini Henrici Laecman (48) pro quo habemus apud Oteey (49) II modios II sextarios II terciarios spelte, emptos a Johanne Pirlin de Villari Episcopi $(50)$ et debentur distribui presentibus in vigiliis $\left(^{a}\right)$.

( $\left.{ }^{a}\right) E$ : écriture de type littera cursiva $\left(X V^{r e} s.\right)$.

(43) Jean de Heers, sous-diacre, appartient au fonds initial de l'obituaire. Il est donc probablement antérieur à 1372. Un Johannes subdiaconus est commémoré le 9 février dans l'O.S.L., éd. MarCHANDisse, p. 19 et n. 101.

(44) Zoticus et ses compagnons (Hiereneus, Jacinctus et Habundus, lequel manque ici), martyrs. Fête : le 10 février.

(45) Sainte Scholastique, vierge. Fête : le 10 février.

(46) Sainte Sotheris, vierge et martyre. Fête : le 10 février.

(47) André d'île, chanoine de Saint-Materne, est cité du $1^{\text {er }}$ juin 1330 au 26 mai 1338. Il était décédé le 15 décembre 1351. Cf. C.S.L., t. 3, pp. 343, 423, 439, 442, 472, 484, 486, $491,508,549$; t. 4, p. 162 ; t. 6, p. 76. - SchoonBrood, Val-Saint-Lambert, t. 1, p. 208. - LaHAYe, Saint-Materne, p. 109. Il est commémoré le 12 février dans l'O.S.L., éd. Marchandisse, p. 21 et n. 113.

(48) Henri Laecman, chanoine (sous-diacre) de Saint-Materne, perçut sa prébende en 1440. Il est cité le 25 avril 1447 et mourut le 11 février 1454. Cf. LaHAYE, Saint-Materne, p. 143. - C.S.L., t. 5, pp. 135, 209.

(49) Othée (pr. Liège, arr. Liège, comm. Awans).

(50) Jean Pirlin de Villers-l'Évêque est cité le 23 septembre 1434, le 27 octobre 1437, le 23 juillet 1454 et le 16 juillet 1457 . Cf. C.S.L., t. 5 , pp. 103, 156; t. 6, pp. 175, 187. 
Item, similiter, pro magistro Jacobo Cappelle (51), I modium.

$\left[8 r^{\circ}\right] \quad$ B idus

(13 février)

Commemoratio Gaufridi Ruppini $\left(^{a}\right)(52)$, canonici Leodiensis, pro quo habemus II modios cum dimidio spelte $\left({ }^{b}\right)$ supra granarium dominorum $\left(^{(}\right)$.

XVIII C XVI kalendas

(14 février)

Marcii (53), Valentini (54), Vitalis, Felicule et Zenonis (55) martyrum. Collecta et missa.

VII D XV kalendas

(15 février)

$\left[8 v^{0}\right]$ E XIIII kalendas

(16 février)

Juliane (56) virginis. Collecta.

(a) subdyaconi, suscrit. - $\left(^{b}\right) B:$ écriture de type littera textualis formata $\left(X I V^{e}-X V^{e}\right.$ s.). - - (c) supra granarium dominorum, ajout d'une main postérieure.

(51) Jacques Capelle, chanoine (diacre) de Saint-Materne. Successeur d'Olivier de Malle ( $\$ 3$ décembre 1427), il commença à percevoir la prébende pleine après l'expiration de l'année de grâce du défunt, le 5 décembre 1428. Il est cité comme exécuteur testamentaire de Jacques de Lovelde, chanoine de Saint-Lambert, le 20 juin 1453, et de Guillaume de Wavera, chanoine de la collégiale Saint-Jean de Liège, le 3 février 1456. Il mourut peut-être le 12 février 1470 (?). Cf. LAHAYE, Saint-Materne, p. 125 . - C.S.L., t. 5, p. 149. - Lahaye, Saint-Jean, t. 1, p. 413.

(52) Gaufroid Rupin, chanoine de Saint-Lambert de 1334 à ca 1361, vice-doyen de Saint-Lambert en 1343. Il possédait un bénéfice dans la région de Poitiers. Il mourut le 10 février 1366. Cf. Сhot-Stassart, p. 203. - Poncelet, Sainte-Croix, t. 1, p. ClXVI. - BerLière, Lettres Clément VI, $\mathrm{n}^{0 \mathrm{~s}} 775,1663,1684$, pp. 253, 618, 624. - JeAN DE HoCSem, Chronique, éd. G. Kurth, Bruxelles, 1927, p. 347. Il est commémoré le 10 février dans l'O.S.L., éd. Marchandisse, p. 20 et n. 105 et le 14 février dans l'ob. Sainte-Croix (I), $\mathrm{f}^{\circ} 124 \mathrm{v}^{\circ}$. - ob. Sainte-Croix (II), $\mathrm{f}^{\circ} 39 \mathrm{v}^{\circ}$. - ob. Sainte-Croix $(I I I), \mathrm{f}^{\circ} 14 \mathrm{v}^{\circ}$.

(53) Saint non identifié.

(54) Saint Valentin, évêque et martyr. Fête : le 14 février.

(55) Saints Vital, Félicole et Zénon, martyrs. Fête : le 14 février.

(56) Sainte Julienne, vierge et martyre. Fête : le 16 février. 
XV F XIII kalendas

(17 février)

IIII G XII kalendas

(18 février)

$\left[9 r^{\circ}\right] \quad$ A XI kalendas

(19 février)

XII B X kalendas

(20 février)

I C IX kalendas

(21 février)

Commemoratio Johannis de Aquis (57), concanonici nostri. VI sextarii spelte apud Villari Episcopi(58) et XII sextarii spelte in granario $\left({ }^{a}\right)$.

$\left[9 v^{o}\right]$

D VIII kalendas

(22 février)

Cathedra Sancti Petri (59). IX lectiones.

IX E VII kalendas

(23 février)

Vigilia.

Commemoratio domini Theoderici Buke $(60)$, presbyteri

(a) E: écriture de type littera cursiva ( $X V^{e}$ s.).

(57) Deux chanoines (sous-diacres) de Saint-Materne portèrent ce nom. L'un, cité dès 1371 et notamment le 30 avril 1374, occupait la seconde place par rang d'ancienneté en 1400. Il est encore cité en 1407 et fut remplacé en 1409-1410 par Jean d'Aix junior, décédé le $1^{\text {er }}$ mars 1415. Cf. LaHaYe, Saint-Materne, p. 147. - C.S.L., 1. 4, p. 508. - SCHOOLMEESTERS, Notice, pp. 45, 47.

(58) Villers-l'Évêque. Cf. note 25.

(59) Chaire de Saint-Pierre. Fête : le 22 février.

(60) Thierry Bucke, chanoine (prêtre) de Saint-Materne, occupait la onzième et dernière place par rang d'ancienneté en 1393. Il fit partie des chanoines qui s'opposèrent au pseudo-élu, Thierry de Hornes ou de Perwez. Il mourut en 1411. Cf. LaHAYE, Saint-Materne, p. 114. - SCHOOLMEesTERs, Notice, p. 33. - ID., Quelques nouveaux documents concernant le schisme de Thierry de Perwez (1406-1408), BulleTin DE LA SocíTÉ DES BIBLIOPHILES LIÉGEOIS, t. 9, 1910, p. 162. 
concanonici nostri, pro quo habemus $\left(^{a}\right)$ II modios spelte $\left(^{b}\right)$.

F VI kalendas

(24 février)

Mathie (61) apostoli. Duplex.

$\left[10 r^{\circ}\right]$ XVII G V kalendas

(25 février)

Commemoratio Symonis Offet (62), concanonici nostri, pro quo habemus VI bonuaria terre arabilis apud Vilarium Episcopi (63) VIII virgis minus ad aniversarium ipsius. Item habemus II modios siliginis ad idem aniversarium. Valet XXII modios spelte. Et si veniant vigiliae in die Cathedre Petri possunt fieri.

VI A IIII kalendas

(26 février)

$\left[10 v^{\circ}\right] \quad$ B III kalendas

(27 février)

XIIII C II kalendas

(28 février)

\section{NOX HABET HORAS XIIII, DIES X}

${ }^{(a)} C$ : écriture de type littera textualis $\left(X V^{e} s.\right) .-\left({ }^{b}\right)$ II modios spelte, ajout d'une main postérieure.

(61) Saint Mat(t)hias (Mathie), apôtre. Fête : le 24 février, les années communes; le 25 février, les années bissextiles.

(62) Simon Offet, chanoine de Saint-Materne, est cité entre 1224 et 1258 . Cf. C.S.L., t. 1, pp. $356,358,549 ;$ t. 3, p. 191 - Poncelet, Hugues de Pierrepont, p. 220 n. 1 . Cuvelier, Val-Benoit, pp. 50,61, 124, 142, 149. Simon Offet est cité comme chanoine de Saint-Martin en 1236 (n. st.), 1247 (n. st.) et 1252. Il y a probablement eu confusion entre Saint-Materne et Saint-Martin (cf. Schoonkrood, Val-Saint-Lambert, t. 1, pp. 50, 70, 80). Simon Offet est commémoré le 23 février dans l'O.S.L., éd. MarChandisSe, p. 27 et n. 148, et le 25 février dans le Polyptyque de Saint-Lambert, pp. 161, 182.

(63) Villers-l'Évêque, cf. note 25. 


\section{$\left[11 r^{\circ}\right]$ MARTIUS PRIMA NECAT, CUIUS SUB CUSPIDE QUARTA EST (64)}

\section{MARTIUS HABET DIES XXXI, LUNA XXX}

III D kalendas

(1 mars)

Commemoratio domini Hermanni de Zanctis (65), presbyteri canonici Leodiensis, pro quo habemus XII sextarios spelte in granario nostro $\left(^{a}\right)$.

E VI nonis

(2 mars)

XI $\quad \mathrm{F} \quad \mathrm{V}$ nonis

(3 mars)

$\left[11 v^{o}\right]$

G IIII nonis

(4 mars)

XIX A III nonis

(5 mars)

Commemoratio domini Johannis Hansange (66), canonici Leodiensis presbyteri, pro quo habemus VIII solidos $X$ denarios bone $\left(^{b}\right)$.

( $\left.{ }^{a}\right) D$ : écriture de type littera cursiva formata $\left(X I V^{e}-X V^{e}\right.$ s. $) .-\left({ }^{b}\right)$ E: écriture de type littera cursiva $\left(X I V^{e}-X V^{e}\right.$ s.).

(64) La forme habituelle est Martis prima necat, cujus sub cuspide quarta est. Cf. Grotefend, Zeitrechnung, t. 1, p. 36. Cf. également note 1.

(65) Cf. note 4.

(66) Maître Jean dit Haensangh ou Hanezanc, de Tongres, cité à partir du 9 avril 1309 comme chanoine de Notre-Dame de Tongres, comme scelleur de l'évêché de Liège de 1323 à 1330 et comme chanoine de la collégiale Sainte-Croix de Liège dès 1327. Il fut pourvu d'une prébende à Saint-Lambert avant le 27 juillet 1338. Il fut aussi coûtre de Dinant et abbé séculier de Thuin (pr. Hainaut, arr. et comm. Thuin). Il mourut le 5 mars 1349. Cf. RenaRdy, Les maitres universitaires, pp. 346-347. Il est commémoré le 2 mars dans l'ob. Sainte-Croix $(I), \mathrm{f}^{\circ} 126 \mathrm{r}^{\circ} .-o b$. Sainte-Croix $(I I), \mathrm{f}^{\circ} 38 \mathrm{v}^{\circ} .-o b$. Sainte-Croix (III), $\mathrm{f}^{\circ} 18 \mathrm{v}^{\circ}$; le 5 mars dans l'O.S.L., éd. Marchandisse, p. 32 et n. 185, - ob. N.-D. Tongres, éd. Paquay, B.S.S.L.L., t. 41, 1927, p. 67. 
Commemoratio domini Henrici Pinte (67), capellani Leodiensis, pro quo habemus IIII sextarios spelte de VI capellanis altaris fundati per dominum Johannem de Machinia (68).

Eodem die commemoratio domini Wilhelmi de Buten, presbyteri nostri concanonici, pro quo habemus VI sextarios spelte apud Opeyum(69) supra unam curtem et domum $\left({ }^{a}\right)$.

$\left[12 r^{\circ}\right] \quad$ C nonis

Perpetue et Felicitatis (70) virginum et martyrum. Collecta.

XVI D VIII idus

Commemoratio Thonardi de Puteo de Aleur (71) et Elyzabeth (72) eius uxoris, parentum Johannis de Aleur (73),

$\left({ }^{a}\right) B$ : écriture de type littera textualis formata $\left(X V^{r}\right.$ s.).

(67) Henri Pinte est cité comme chapelain de la collégiale Saint-Denis de Liège, comme collecteur, récepteur et computateur des biens du Val-Benoît ainsi que comme maire de la cour des biens dépendant du Val-Benoît, le 26 janvier et le $1^{\text {er }}$ juin 1327 , le 19 juin 1330 et le 18 septembre 1331. Cf. Cuvelier, Val-Benoît, pp. 385, 386, 407, 414, 415. Il est également cité comme chapelain de Saint-Lambert, le 27 mai 1344. Cf. Schoonbroodt, Val-Saint-Lambert, t. 1, p. 195. - FaIron, Regestes, t. 1, p. 344.

(68) Il s'agit probablement de Jean de Marlines, chanoine de Saint-Lambert en 1315 et chanoine de la collégiale Saint-Paul de Liège. Il est décédé le 18 janvier 1330. Cf. Chot-STASSART, p. 179. Il est commémoré le 17 novembre dans l'O.S.L., éd. MarchanDisse, p. 159. Pour son épitaphe à Saint-Lambert, cf. Naveau-Poullet, t. 1, p. 14.

(69) Probablement Oupeye (pr. et arr. Liège, comm. Oupeye).

(70) Saintes Perpétue et Félicité, martyres. Fête : le 7 mars.

(71) Thonard du Puits d'Alleur est cité comme homme allodial le 20 juin 1388. Cf. VAN Der Made, Guillemins, pp. 82-83. Il est décédé en 1392, selon son épitaphe à Alleur. Cf. Naveau-Poullet, t. 2, p. 257 et n. 2.

(72) Élisabeth, épouse du précédent, est décédée le 8 mars 1403. Elle fut inhumée à l'église Saint-Servais de Liège. Cf. Naveau-Poullet, t. 2, p. 257 et n. 2.

(73) Jean d'Alleur, chanoine (prêtre) de Saint-Materne, est fréquemment cité notamment comme fondé de pouvoir de ses collègues, chanoines, entre le 22 juin 1393 et le 
nostri concanonici, pro quo habemus apud Engys (74) IIII sextarios spelte $\left({ }^{a}\right)$.

Item, eadem die, commemoratio Johannis de Alleur, condam nostri canonici et dyaconi, filii eorumden, pro quo habemus apud Harstappe (75) III modios spelte hereditarie. Item I modium spelte hereditarie aput Engis (76). Item XXI solidos III denarios III copeys bone. Et debent fieri II candele de dimidia libra cere quae ardere debent in capella Sancti Materni (77) ab inicio vigiliarum usque ad finem misse die sequentis continue $\left(^{(}\right)$.

Commemoratio domini Johannis de Perweis $\left({ }^{b}\right)(78)$ ac omnium benefactorum suorum, pro quibus habemus $I$ modium spelte aput Engis $\left(^{a}\right)(79)$.

$\left.{ }^{a}\right) B$ : écriture de type littera textualis formata $\left(X V^{e} s.\right) .-\left({ }^{b}\right)$ canonici nostri, suscrit.

11 juin 1437. Il meurt le 5 juin 1443. Cf. LaHaYe, Saint-Materne, p. 128. - C.S.L., t. 5, pp. 9, 17, 57, 76, 90, 105, 108, 113 ; t. 6, pp. 157, 160, 164, 171. - ScHOONBRoODT, Saint-Martin, p. 125. - SChOOLmeEsters, Notice, p. 48. Il n'est pas impossible que l'un des ecclésiastiques portant le nom de Jean de Pu(i)che (c'est-à-dire du Puits, patronyme $\mathrm{du}$ père de Jean d'Alleur) et apparaissant fréquemment dans les textes puisse être rapproché de Jean d'Alleur, chanoine de Saint-Materne, parfois dit de Puiche. $\grave{A}$ leur propos, cf. Schoonbrood, Val-Saint-Lambert, t. 1, pp. 208, 246. - VAN Der MAdE, Guillemins, pp. 55, 65, 66, 67-68, 70-71, 75-76, 77. - C.S.L., t. 4, p. 642. - Cuvelier, Val-Benoût, pp. 464, 496, 657. - Poncelet, Sainte-Croix, t. 1, pp. 245, 394, 436. - Lahaye, Saint-Jean, t. 1, pp. 235, 237, 261.

(74) Engis (pr. Liège, arr. Huy, comm. Engis).

(75) Herstappe (pr. Limbourg, arr. Tongres, comm. Tongres).

(76) Cf. note 74 .

(77) Sur la chapelle desservie par les chanoines de Saint-Materne, cf. introduction, chapitre 1, p. 13.

(78) Jean de Perwez, chanoine (diacre) de Saint-Materne, est cité le 4 mai 1412. Son testament fut approuvé le 23 février 1447. Il était décédé le $1^{\text {er }}$ mars 1449 (dispositions testamentaires à cette date). Cf. LAHAYE, Saint-Materne, p. 132. - C.S.L., t. 5, p. 140 ; t. 6 , p. 163.

(79) Engis. Cf. note 74. 
Commemoratio domini Eustatii de Langdris (80), presbyteri concanonici nostri, pro quo habemus $X$ sextarios $I I$ tertiarios spelte $\left({ }^{b}\right)$.

XIII G V idus

(11 mars)

Commemoratio Marie $\left({ }^{c}\right)$, ancille domini Johannis Borgondi $(81)$. $X$ solidi bone $\left({ }^{d}\right)$.

II A IIII idus

(12 mars)

Gregorii (82) pape et confessoris. Duplex.

Commemoratio domini Petri de Kirpeck (83), dyaconi canonici Leodiensis, pro quo habemus I modium spelte videlicet IIII sextarios apud Othee (84) et IIII sextarios apud Fexhe (85), de XX sextariis a Johanne delle Soxhe (86) emptis $\left({ }^{(}\right)$.

$\left({ }^{a}\right)$ En marge extérieure, < Eustatius $>$ de Langdris, canonicus noster, $<\mathrm{X}>$ sextarii spelte, aj. - $\left(^{b}\right)$ E: écriture de type littera cursiva $\left(X I V^{e}-X V^{p}\right.$ s.). - (c) de Alledes, suscrit. - $\left.{ }^{d}\right) D$ : écriture de type littera cursiva formata $\left(X I V^{p}-X V^{p}\right.$ s. $) .-\left({ }^{e}\right) B:$ écriture de type littera textualis formata $\left(X I V^{r}-X V^{r} s.\right)$.

(80) Eustache de Langdris, chanoine (prêtre) de Saint-Materne, est cité en 1376 et le 12 septembre 1384. Il est décédé le 10 mars 1392. C'est peut-être déjà lui qui est cité sous le nom de Stassines de Landris et sous l'appellation de clerc le $1^{\text {et }}$ septembre 1353. Cf. LAHAYE, Saint-Materne, pp. 110, 121. - C.S.L., t. 4, p. 178. - CuvELIER, Val-Benoit, p. 343.

(81) Un certain Josse le Bourguignon, chanoine prébendé de Saint-Materne est cité comme homme dele Cyse Dieu le $1^{\text {er }}$ mars 1372 (cf. C.S.L., t. 4, p. 492). Peut-être faut-il voir en ce personnage le chanoine ici commémoré ? Quoi qu'il en soit, Jean le Bourguignon, chanoine prébendé de Saint-Materne, était déjà décédé le 21 janvier 1375. Cf. Tinon, Lettres de Grégoire XI, t. 3, n 3004 bis, p. 15.

(82) Saint Grégoire $I^{\text {er }}$ le Grand, pape, conf. Fête : le 12 mars.

(83) Pierre de Kirpech obtint une expectative de prébende à Saint-Lambert le 22 mai 1342 et à la collégiale Saint-Paul de Liège, le 29 septembre 1342. Il fut chanoine de Saint-Séverin de Cologne (1342) et est encore cité comme chanoine de Saint-Lambert le 28 novembre 1358. Cf. Chot-Stassart, p. 168. - C.S.L., t. 6, p. 113. - Berlière, Suppliques Clément VI, $\mathrm{n}^{\mathrm{os}} 16,222,1541$, pp. 5, 46, 415. - Berrik̀re, Lettres Clément VI, $\mathrm{n}^{\text {os }} 15,525,575$, pp. $5,169,186$. Il est commémoré le 14 mars dans l'O.S.L., éd. MARCHANDISSE, p. 35 et n. 204.

(84) Othée (pr. Liège, arr. Liège, comm. Awans).

(85) Probablement Fexhe-Slins (pr. Liège, arr. Liège, comm. Juprelle).

(86) Cf. note 37. 
$\left[13 r^{\circ}\right] \quad$ B III idus

(13 mars)

X C II idus

(14 mars)

D idus

(15 mars)

$\left[13 v^{v}\right]$ XVIII E XVII kalendas aprilis

(16 mars)

VII F XVI kalendas

(17 mars)

Gertrudis (87) virginis. IX lectiones $\left(^{a}\right)$.

Commemoratio confratris nostri domini Mairsu a quo habemus $C$ florenos colonienses et fient due commemorationes ut in qualibet commemoratio distribuentur inter presentes III florinos colonienses $\left({ }^{b}\right)$.

G XV kalendas

(18 mars)

Commemoratio Stassini dicti Stasson, incisoris vestium, pro quo habemus I modium spelte supra.

$\left[14 r^{\circ}\right]$ XV A XIIII kalendas

(19 mars)

IIII B XIII kalendas

(20 mars)

C XII kalendas

(21 mars)

Benedicti (88) abbatis et confessoris. IX lectiones. XVI

$\left({ }^{a}\right)$ duplex, suscrit. $-\left({ }^{b}\right)$ E: écriture de type littera cursiva $\left(X I V^{p}-X V^{p}\right.$ s.).

(87) Sainte Gertrude, vierge, abbesse. Fête : le 17 mars.

(88) Saint Benoît de Nursie. Fête : le 21 mars. 
$\left[14 v^{\circ}\right]$ XII D XI kalendas

(22 mars)

I E X kalendas

(23 mars)

Commemoratio Oode mulieris, quondam matris magistri Helye (89), concanonici nostri, pro qua habemus $\mathrm{V}$ modios spelte et II sextarios super quandam terram jacentem apud Villarium Episcopi (90).

F IX kalendas

(24 mars)

Commemoratio domini Petri Kokin (91), concanonici nostri, pro quo habemus I marcham bone monete $\left({ }^{a}\right)$.

$\left[15 r^{\circ}\right]$ IX G VIII kalendas

(25 mars)

Annuntiatio Domini (92). Totum duplex.

A VII kalendas

(26 mars)

XVII B VI kalendas

(27 mars)

Resurrectio Domini (93). Totum duplex $\left({ }^{b}\right)$.

$\left({ }^{a}\right) B$ : écriture de type littera textualis formata $\left(X I V^{e}-X V^{e} s.\right) .-\left({ }^{b}\right) \dot{A}$ côté, en chiffres arabes et l'un en-dessous de l'autre: 1407, $1334 \mathrm{~B}$.

(89) Cf. note 41.

(90) Villers-l'Évêque. Cf. note 25.

(91) Pierre Kokin, chanoine (sous-diacre) de Saint-Materne, est décédé le 5 mars 1394. Il est cité le 11 janvier 1381 comme tenant de la cour de la collégiale Saint-Denis de Liège et le 15 mars 1393 comme tenant de la cour jurée de la Cathédrale. Cf. LAHAYE, Saint-Materne, p. 143. - C.S.L., t. 4, p. 591 ; t. 5 , p. 8.

(92) Annonciation. Fête : le 25 mars.

(93) Résurrection du Christ. Fête : le 27 mars, à Liège. Cf. Grotefend, Zeitrechnung, t. 2, p. 106. 
$\left[15 v^{o}\right]$ VI C V kalendas

(28 mars)

Commemoratio domini Johannis de Bovingne (94), decani Sancte Crucis, pro quo habemus apud Freris (95) VII solidos fortes $\left({ }^{a}\right)$.

D IIII kalendas

(29 mars)

XVIII

XIIII E III kalendas

(30 mars)

VII

III F II kalendas

(31 mars)

NOX HABET HORAS XII, DIES XII APRILIS DECIMO EST, UNDENO A FINE MINATUR (96)

\section{APRILIS HABET DIES XXX, LUNA XXIX}

G kalendas

Commemoratio domini Hermanni de Zanctis (97), presby-

$\left({ }^{a}\right)$ L'abréviation fort est complétée par es, suscrit et d'une autre main. Suit un mot, probablement fortes, barré. En marge intérieure: Notum sit quod in die palmarum non fiunt vigilie, rubriqué.

(94) Jean de Bouvignes, doyen de la collégiale Sainte-Croix de Liège de 1304 à 1316. Il mourut le 4 mars 1316. Cf. PonCELET, Sainte-Croix, t. 1, pp. LIX, LX, XCVI, 84-86, 89, 97. Le 19 juillet 1317, le chapitre de Saint-Lambert fait savoir que Godefroid de Bouvignes donne une rente à Vreeren (cf. note suivante) aux chanoines de Saint-Materne, pour le repos de l'âme de son frère, Jean de Bouvignes. Cf. C.S.L., t. 3, pp. 172-173. Il est commémoré le 4 mars dans l'ob. Sainte-Croix $(I), \mathrm{f}^{\circ} 126 \mathrm{r}^{\circ}$. - ob. Sainte-Croix $(I I I), \mathrm{f}^{\circ} 18 \mathrm{v}^{\circ}$.

(95) Vreeren (pr. Limbourg, arr. Tongres, comm. Tongres).

(96) La forme habituelle est Aprilis decima et undena a fine minantur. Cf. GROTEFEND, Zeitrechnung, t. 1, p. 36. Cf. également, ici-même, n. 1.

(97) Cf, note 4. 
teri canonici Leodiensis, pro quo habemus I modium spelte in granario nostro $\left({ }^{a}\right)$.

XI A IIII nonis

B III nonis

(3 avril)

$\left[16 v^{\circ}\right]$ XIX C II nonis

(4 avril)

Ambrosii (98) episcopi et confessoris. Duplex.

XII

VIII D nonis

(5 avril)

XVI E VIII idus

(6 avril)

$\left[17 r^{\circ}\right] \quad$ V $\quad$ F VII idus

(7 avril)

IX

G VI idus

(8 avril)

Commemoratio domini Reneri de Wilhe (99), presbyteri concanonici nostri, pro quo habemus II sextarios spelte $\left({ }^{b}\right)$.

XIII A V idus

( 9 avril)

Marie Egyptyace (100). IX lectiones.

( $\left.{ }^{a}\right)$ D: écriture de type littera cursiva formata $\left(X I V^{e}-X V^{e}\right.$ s.). $-\left(b^{b}\right) D$ : écriture de type littera cursiva formata $\left(X I V^{e}-X V^{*} s\right.$.). En marge extéricure, $\operatorname{Re}<$ nerus de $>$ Vilhe $\mathrm{p}<$ resbyter concanonicus $>$ noster II $<$ sextarii spelte $>$.

(98) Saint Ambroise, évêque, conf. Fête : le 4 avril.

(99) Renier de Vilhe, chanoine (prêtre) de Saint-Materne, est cité du $1^{\text {er }}$ octobre 1360 à 1375. Il était décédé en 1383 et fut remplacé par Guillaume de Ryckel ou de Richelle (cf. note 231). Cf. Lahaye, Saint-Materne, p. 117. - C.S.L., t. 4, pp. 328, 508, 512 n. 3. (100) Sainte Marie l'Égyptienne. Fête: le 9 avril. 
Commemoratio Enoc (101), sacerdotis et concanonici nostri, pro quo habemus II bonuaria terre apud Vileri Episcopi (102) reddentia VI modios.

$\left[17 v^{\circ}\right]$ II $\quad$ B IIII idus

(10 avril)

C III idus

(11 avril)

Leonis (103) pape et confessoris. Collecta.

Commemoratio domini Hugonis (104), episcopi, pro quo habemus VIII bonuaria terre et I jornale apud Voteme (105) reddentia XVI modios spelte quos debent heredes Johannis Tasseron.

$\mathrm{X} \quad \mathrm{D}$ II idus

$\left[18 r^{\circ}\right] \quad$ E idus

(13 avril)

Commemoratio Galteri de Hesdin (106), concanonici nostri, pro quo habemus VI solidos de censu ultra

(101) Enoc, chanoine de Saint-Materne, est cité comme témoin dans des actes datés de 1215. Cf. Paquay, Cartulaire N.-D. Tongres, t. 1, pp. 66, 68-69. Il est commémoré le 8 avril comme presbyter, f.n., dans l'ob. N.-D. Tongres, éd. PaQuaY, B.S.S.L.L., t. 41, 1927, p. 73 ; le 9 avril dans l'O.S.L., éd. MarChandisSe, p. 48 et n. 287, et dans le Polyptyque de Saint-Lambert, p. 185.

(102) Villers-l'Évêque. Cf. note 25.

(103) Saint Léon $\mathrm{I}^{\mathrm{er}}$, pape, conf. Fête : le 11 avril.

(104) Hugues de Pierrepont, évêque de Liège (5 mars $1200-12$ avril 1229). Cf. E. I. Strubbe-L. Voet, De chronologie van de middeleeuwen en de moderne tijden in de Nederlanden, Anvers-Amsterdam, 1960, p. 282. - Poncelet, Hugues de Pierrepont, p. XII. Il est commémoré dans de nombreux documents nécrologiques. On trouvera la liste de ceux-ci dans l'O.S.L., éd. Marchandisse, p. 49 et n. 295. Pour son épitaphe à Saint-Lambert, cf. Naveau-Pouller, t. 1, p. 1.

(105) Vottem (pr. Liège, arr. Liège, comm. Herstal).

(106) Maître Gauthier de Hesdin (France, dép. Pas-de-Calais, Arras), chanoine de Saint-Materne, fit, le 12 octobre 1231, une importante donation au chapitre auquel il appartenait. Cf. RenARdy, Les maîtres universitaires, p. 228. - C.S.L., t. 1, pp. 284-286. 
Mosam (107) et IX solidos de domo domini Marchi in Sabuleto (108) et IIII solidos et VI denarios apud Vinetum (109). Item dedit nobis domum suam in Sabuleto pro qua habemus VI modios siliginis super III bonuaria terre apud Horpale (110). De quibus VI modiis habemus II modios in anniversario suo cum denariis predictis.

\section{F XVIII kalendas maii}

Tyburtii et Valeriani (111) martyrum. Collecta $\left({ }^{a}\right)$.

Commemoratio Henrici sacerdotis pro quo habemus I modium siliginis in granario nostro.

Item, eodem die, commemoratio Johannis de Marcella, dyaconi canonici Sancti Materni et suorum parentorum $\left({ }^{b}\right)$. I modius spelte in granario nostro $\left(^{c}\right)$.

Eadem die commemoratio magistri Anthonii de Fiez (112), dyaconi canonici Leodiensis, pro quo habemus

$\left(^{a}\right)$ et missa, suscrit. - $\left.{ }^{b}\right)$ Ces mots, rédigés par la main initiale, ont été biffés puis suscrits par la main qui rédigea la notice d'Antoine de Fize. $\left.-{ }^{c}\right)$ Ces mots ont été ajoutés par la main qui a rédigé la notice d'Antoine de Fize. Cf. note suivante.

(107) Outremeuse, quartier de Liège. Cf. GoberT, Les rues de Liège, t. 4, pp. 382-384.

(108) Sauvenière, quartier de Liège. Cf. GoberT, Les rues de Liège, t. 5, pp. 284-302.

(109) Vivegnis, lieu-dit à Liège. Cf. GoberT, Les rues de Liège, t. 5, pp. 625-630.

(110) Horpmaal, commune de Heers (pr. Limbourg, arr. Tongres, comm. Heks).

(111) Saints Tiburce, Valerien et Maximin, martyrs. Fête : le 14 avril.

(112) Antoine de Fize possédait une prébende à Notre-Dame de Tongres avant le 29 août 1359 et reçut une prébende à la collégiale Saint-Jean de Liège le 22 janvier 1362 . Le 14 février 1363, il devint chanoine prébendé à Saint-Lambert et, pour ce faire, renonça à la prébende qu'il détenait à Tongres, le 4 juillet 1363 . Il devint prévôt de la collégiale Sainte-Croix de Liège le 16 février 1363 et est cité comme chanoine de Saint-Lambert ainsi que de Tongres en 1364, 1365, 1366, 1368 et 1375 . Le 26 juillet 1371 , il reçut confirmation de la prévôté de la collégiale Saint-Denis de Liège et est cité comme official en 1373 et en 1374. Cf. Сhot-Stassart, p. 150. - Hemricourt, t. 1, p. 448 ; t. 2, p. 257. - C.S.L., t. 4, pp. 427, 433, 460, 512, 520, 657 ; t. 5, p. 3 ; t. 6, pp. 128, 134. - PonCELET, Sainte-Croix, t. 1, pp. LI, 253, 286, 299. - LAHAYE, Saint-Jean, t. 1, p. LXXII. Paquay, Cartulaire N.-D. Tongres, t. 1, pp. 458, 483, 487, 489, 504. - SCHOONBroodT, 
I modium spelte supra dimidium bonuarium terre jacentem in territorio de Fiez (113) $\left.{ }^{a}\right)$.

VII G XVII kalendas

Commemoratio magistri Helie $\left(^{b}\right)(114)$, bone memorie, qui legavit nobis II bonuaria terre site apud Villare Episcopi (115) pro quibus habemus annuatim VI modios spelte, qui etiam (c) legavit calicem, pixidem, eburneam albam et casulam et cetera quae ad cultum altaris pertinent.

XV B XV kalendas

Ursmari (116) episcopi et confessoris. Commemoratio.

$\left({ }^{a}\right) C$ : écriture de type littera textualis $\left(X I V^{e}-X V^{e} s.\right) .-\left({ }^{b}\right)$ presbyteri concanonici nostri, suscrit. $-\left({ }^{c}\right)$ nobis, biffé.

Val-Saint-Lambert, t. 1, p. 239. - Cuvelier, Val-Benoît, pp. 517, 520, 537, 570, 590, 595.

- Fierens, Suppliques Urbain V, $\mathrm{n}^{\text {os }} 644,912-913,1019,1041,1400,1514,1521$, pp. 200-201, 309, 353, 361-362, 527, 568, 573. - FIERENS, Lettres Urbain V, t. 1, $\mathrm{n}^{\text {os }} 403$, $635,645,730,847,855,1311,1563,1592,1909$, pp. 137, 228, 236, 301, 358, 360, 593, 710, 723,888 ; t. 2 , n ${ }^{\circ} 2612$, p. 368 . - BerLIÈre, Suppliques Innocent VI, $\mathrm{n}^{\text {os }} 1343,1536$, pp. 548, 626. - TıноN, Lettres Grégoire XI, t. 1, $\mathrm{n}^{\text {os }} 347,1032,1071$, pp. 167, 427, $447-448 ;$ t. $3, n^{\circ} 3596$, p. 308. - É. SCHOOLMEESTERS, Les officiaux des évêques de Liège jusqu'au XVI siècle, Leodium, t. 7, 1908, p. 123. Selon Tinon, Lettres Grégoire XI, t. 3, $\mathrm{n}^{\circ} 3596$, p. 308, Antoine de Fize est décédé entre le 2 février et le 27 avril 1376. L'ob. Saint-Materne permet de préciser cette date: le 14 avril 1376. Il est commémoré le 14 avril dans l'ob. N.-D. Tongres, éd. PAQuaY, B.S.S.L.L., t. 41, 1927, p. 75. Cf, la notice consacrée à Gilles de Fize, note 10 .

(113) Fize-le-Marsal (pr. Liège, arr. Waremme, comm. Crisnée).

(114) Cf. note 41.

(115) Villers-l'Évêque, cf. note 25.

(116) Saint Ursmer, abbé (évêque), conf. Fête: le 18 avril. 
$\left[19 r^{\circ}\right] \quad$ D XIII kalendas

(19 avril)

XII E XII kalendas

(20 avril)

Commemoratio Jacobi dicti Malechair de Hoyo (117), concanonici nostri subdyaconi, pro quo habemus XII sextarios spelte apud Pucey (118).

I $\quad$ F XI kalendas

(21 avril)

Commemoratio domini Johannis (119), quondam decani Sancti Lamberti, bone memorie, pro quo habemus I bonuarium terre apud Genefe (120). Nobis in aniversario ipsius reddentur III modii spelte.

$\left[19 v^{\circ}\right] \quad$ G X kalendas

(22 avril)

IX A IX kalendas

(23 avril)

Georgii (121) martyris. Duplex. Adalberti (122) episcopi et martyris. Collecta.

(117) Jacques (Jakemars) dit Malechair de Huy est chanoine de la Petite-Table le 17 avril 1330. Il est cité comme chanoine de Saint-Materne du 13 décembre 1337 - de Saint-Martin, est-il dit erronément - au 12 décembre 1350. Il était décédé le 28 mai 1356. Il fut également chanoine et chantre de Notre-Dame de Huy ainsi que chanoine de la collégiale Saint-Paul de Liège. Cf. C.S.L., t. 3, pp. 423 n. 4, 603 et n. 2 ; t. 4, pp. 86, 97, 99, 102, 103, 136, 238. - Lahaye, Saint-Jean, t. 1, p. 189. - Poncelet, F.A.M., p. 439. - PONCelet, F.E.M., p. 234. - FAYEN, Lettres Jean XXII, t. 2, n ${ }^{\circ} 2707$, p. 386. - Joris, Ville de Huy, p. 376. - Génicot, Les chanoines de Huy, p. 71. II est commémoré le 22 février dans l'ob. N.-D. Huy, éd. Deckers-Renardy, pp. 45, 195, 251, et le 20 avril dans l'ob. Neufmoustier, éd. Closset, t. 1, p. 21 ; t. 2 , p. 72.

(118) Pousset (pr. Liège, arr. Waremme, comm. Remicourt) ou Poucet (pr. Liège, arr. Waremme, comm. Hannut).

(119) Jean de Moregny, chanoine de Saint-Lambert dès 1215, archidiacre de Brabant (1224-1225), prévôt de la collégiale Saint-Jean de Liège (de 1229 à 1244), doyen de Saint-Lambert (de 1229 à 1253) et écolâtre de la collégiale Saint-Denis de Liège. Il est décédé en 1253. Cf. Chot-Stassart, pp. 95-96. - Paquay, Cartulaire N.-D. Tongres, t. 1, p. 69. É. SCHoOlmeESTERS, Jean de Moregny et la confraternité de Saint-Luc, LeOdium, t. 9, 1910, pp. 37-42. - ID., Jean de Moregny, LeoDium, t. 10, 1911, pp. 135-136. Il est commémoré le 21 avril dans l'O.S.L., éd. MarCHANDisse, p. 54 et n. 333 (quondam decanus n.). - Polyptyque de Saint-Lambert, p. 187. - ob. Saint-Denis, $\mathrm{f}^{\circ} 7 \mathrm{v}^{\circ}$ (comme écolâtre de Saint-Denis).

(120) Jeneffe-en-Hesbaye (pr. Liège, arr. Waremme, comm. Donceel).

(121) Saint Georges, martyr. Fête : le 23 avril.

(122) Saint Adalbert, évêque, martyr. Fête : le 23 avril. 
Commemoratio magistri Nicholai de Turre (123), capellani huius ecclesie, pro quo habemus VI solidos bone supra domum suam sitam ante ecclesiam Sancti Thome apostoli (124).

Eodem die, commemoratio Katherine, sororis domini quondam Johannis de Ensivaiz (125), pro qua habemus $X$ solidos bone in censu domorum nostrarum in Gerardria (126) et Andree, fratris eorumdem, pro quo habemus $X X$ solidos bone supra domum de Caliga (127) in Foro Leodiensis sitam iuxta gradus ecclesie $\left(^{a}\right)$.

B VIII kalendas

(24 avril)

$\left[20 r^{\circ}\right]$ XVII C VII kalendas

(25 avril)

Marci (128) evangeliste. Duplex. Floreberti(129) episcopi et confessoris. Collecta.

D VI kalendas

(26 avril)

E V kalendas

(27 avril)

$\left({ }^{a}\right)$ E : écriture de type littera cursiva $\left(X I V^{c}-X V^{e}\right.$ s.).

(123) Le magister ici mentionné est probablement postérieur à 1372. Les maîtres prénommés Nicolas cités par RENARDY, Les maîtres universitaires, pp. 397, 403, sont, semble$\mathrm{t}$-il, trop anciens.

(124) Sur l'église Saint-Thomas, à Liège, cf. Gobert, Les rues de Liège, t. 5, pp. 430-434. - Lahaye, Paroisses, p. 14 et passim.

(125) Jean de Enzivaux est cité comme chanoine de la Petite-Table le 12 mai 1352. Il est également mentionné comme chanoine de Saint-Materne le $1^{\text {er }}$ octobre 1360 . Il était décédé le 30 avril 1374. Cf. Lahaye, Saint-Materne, p. 109. - C.S.L., t. 4, pp. 160, 328, 508 .

(126) Gérardrie, lieu-dit à Liège. Cr. GoBfRT, Les rues de Liège, t. 3, pp. 122-127. Sur les maisons possédées en Gérardrie par le chapitre de Saint-Materne, cf. introduction, chapitre 1, pp. 13-14.

(127) Selon C.S.L., t. 5, p. 4, la maison delle Chausse (Chache, Chauche) se trouvait sur le marché de Liège, faisant l'angle de la rue de la Chapelle. Il s'agit probablement de l'actuelle rue Chapelle-des-clercs, laquelle est située près de Gérardrie (cf. note précédente).

(128) Saint Marc, évangéliste. Fête : le 25 avril.

(129) Saint Florebert, évêque, conf. Fête : le 25 avril. 
VI D VI kalendas

(26 avril)

E V kalendas

(27 avril)

$\left[20 v^{0}\right] \quad$ XIIII F IIII kalendas

(28 avril)

Translatio Sancti Lamberti (130). Totum duplex. Vitalis (131) martyris. Collecta.

III G III kalendas

(29 avril)

Petri (132) martyris de ordine predicatorum. Collecta.

Commemoratio domini Jacobi de Louz (133), dyaconi et canonici Leodiensis, pro quo habemus III modios spelte supra XXI vergas magnas terre apud Othey (134).

A II kalendas

(30 avril)

NOX HABET HORAS X, DIES XIIII

(130) Translation de saint Lambert. Fête : le 28 avril.

(131) Saint Vitalis, martyr. Fête : le 28 avril.

(132) Saint Pierre de Vérone, prédicateur en Lombardie, nouveau martyr, $\uparrow 1252$, canonisé en 1253. Fête : le 29 avril.

(133) Jacques de Looz, chanoine de Saint-Lambert et prévôt de la collégiale Saint-Denis de Liège dès 1302. Il mourut le 29 avril 1338. Cf. Chot-Stassart, p. 173. HemricourT, t. 2, p. 292. - Fairon, Regestes, t. 1, p. 251 - C.S.L., t. 6, pp. 83, 323. Diverses dispositions testamentaires sont consignées dans C.S.L., t. 3, pp. 524-526, 555. - Bormans, Saint-Denis, pp. 35, 96, 99, 110. - É. Poncelet, Les maréchaux d'armée de l'évêché de Liège, B.I.A.L., t. 32, 1902, pp. 326-327. Il est commémoré le 28 avril dans l'O.S.L., éd. Marchandisse, p. 58 et n. 362. Pour son épitaphe à Saint-Lambert, cf. Naveau-Poullet, t. 1, p. 54.

(134) Othée. Cf. note 84. 
$\left[21 r^{0}\right] \quad$ TERCIUS IN MAIO LUPUS EST ET SEPTIMUS ANGUIS

\section{MAIUS HABET DIES XXXI, LUNA XXX}

XI B kalendas

(1 mai)

Philippi (135) et Jacobi (136) apostolorum. Duplex. Walburgis (137) virginis. Collecta.

Commemoratio magistri Walteri de Morseilh(138), concanonici nostri et presbyteri, pro quo habemus III modios spelte in granario nostro.

Commemoratio domini Hermanni de Zanctis (139), presbyteri canonici Leodiensis, pro quo habemus in granario nostro I modium spelte $\left({ }^{a}\right)$.

C VI nonis

XIX D V nonis

Inventio Sancte Crucis (140). Totum duplex. Alexandri, Eventii (141) et Iuvenalis (142) martyrum. Collecta.

(a) D : écriture de type littera cursiva formata $\left(X I V^{e}-X V^{e}\right.$ s.).

(135) Saint Philippe, apôtre, martyr. Fête : jadis, le $1^{\text {er }}$ mai. Depuis 1956 , fềte : le 11 mai.

(136) Saint Jacques le Mineur, apôtre, martyr. Fête : jadis, le $1^{\text {er }}$ mai, avec saint Philippe. Depuis 1956, fête : le 11 mai.

(137) Sainte Walburge, vierge, abbesse. Fête : le $1^{\text {er }}$ mai.

(138) Cf. note 3.

(139) Cf. note 4 .

(140) Invention de la Sainte-Croix. Fête : le 3 mai.

(141) Saint Alexandre I ${ }^{\text {er }}$, pape. Fête : le 3 mai, avec Eventius et Theodolus, martyrs.

(142) Saint Juvénal, martyr (évêque, conf.). Fête : le 3 mai. 
$\left[21 v^{0}\right]$ VIII E IIII nonis

(4 mai)

F III nonis

(5 mai)

XVI G II nonis

(6 mai)

Johannis (") ante Portam latinam (143). III lectiones.

$\left[22 r^{\circ}\right] \mathrm{V} \quad$ A nonis

(7 mai)

Domiciani (144) episcopi et confessoris. IX lectiones.

B VIII idus

(8 mai)

XIII C VII idus

(9 mai)

$\left[22 v^{\circ}\right]$ II D VI idus

(10 mai)

Gordiani et Epymachi (145) martyrum. Collecta.

Commemoratio Ade (146), dyaconi concanonici nostri, pro quo habemus I bonuarium terre situm apud Geneffe (147). Nobis in aniversario suo reddentur III modii spelte.

E V idus

Gengulphi (148) martyris. Commemoratio.

(c) apostoli, suscrit.

(143) Saint Jean devant la porte d'Arseyn. Fête : le 6 mai.

(144) Saint Domitien, évêque, conf. Fête : le 7 mai.

(145) Saints Gordien et Épimaque, martyrs. Fête : le 10 mai.

(146) Adam, chanoine de Saint-Materne, possédait une maison à Jeneffe-en-Hesbaye. Il est cité et est commémoré dans le Polyptyque de Saint-Lambert, pp. 162, 188, mentionné le 9 mai dans l'O.S.L., éd. MarCHANDisSE, p. 63 et n. 386.

(147) Jeneffe-en-Hesbaye. Cf. note 120.

(148) Saint Gengulphe (Gingolf, Gengoul), martyr. Fête : le 11 mai. 
Nerei et Achillei (149) martyrum. Collecta.

$\left[23 r^{0}\right] \quad$ G III idus

Servatii (150) episcopi et confessoris. Duplex. Marie ad martyres (151). Collecta.

Commemoratio domini Johannis de Fiez (152), canonici Leodiensis presbyteri, pro quo habemus I modium spelte supra bona Colardi Datonghe $\left({ }^{a}\right)$.

VII B idus

Commemoratio domini Anthonii de Nucerio $\left({ }^{b}\right)$ pro quo habet quilibet XII denarios, presbyter XII denarios, et cle-

${ }^{(a)} C$ : écriture de type littera textualis $\left(X I V^{e}-X V^{e} s.\right) .-\left({ }^{b}\right)$ presbyteri, suscrit.

(149) Saints Nérée et Achilée (auxquels s'ajoute Pancrace), martyrs. Fête : le 12 mai.

(150) Saint Servais, évêque, conf. Fête : le 13 mai.

(151) Sainte Marie ad martyres. Fête : le 13 mai.

(152) Jean de Fize était au service de la cathédrale de Liège depuis 24 ans et détenait quelques petits bénéfices, le 15 février 1354. Il est cité comme chanoine de la collégiale Saint-Jean l'Évangéliste à Liège de 1357 à 1360 et comme chanoine de Saint-Lambert dès 1363. Il semble qu'il faille distinguer le chanoine de Saint-Lambert, qui était décédé le 23 mai 1373, d'un autre Jean de Fize, chanoine de Saint-Pierre, cité de 1365 à 1382 et décédé avant le 28 avril 1384. Cf. Сhot-Stassart, p. 150. - Hemricourt, t. I, p. 448 ; t. 2, p. 257. - C.S.L., t. 4, pp. 374, 433, 490, 657. - LaHAYE, Saint-Jean, t. 1, pp. LXXII, 235, 247. - Poncelet, Saint-Pierre, pp. LXII, 75, 85, 90. - Poncelet, Sainte-Croix, t. 1, p. 255. - Cuvelier, Val-Benoit, pp. 537, 555, 772. - BerLière, Suppliques Innocent VI, $\mathrm{n}^{\mathrm{os}} 409,526,928,1060$, pp. 166, 220, 387, 439. - TiHON, Lettres Grégoire $X I$, t. 1, no 694, p. 293 ; t. 2, no 2146, pp. 325-326. - G. DESPY, Lettres d'Innocent VI (1352-1362), t. 1 (1352-1355), Analecta Vaticano-BelgiCa, t. 17, Bruxelles, $1953, \mathrm{n}^{\circ} 627$, p. 215. 
ricus VI denarios, et canonicus habens prebendam Banou (153) prefertur in missa dicenda $\left(^{a}\right)$.

IIII E XV kalendas

$\left[24 r^{\circ}\right] \quad$ F XIIII kalendas

XII G XIII kalendas

(20 mai)

I A XII kalendas

(21 mai)

$\left[24 v^{\circ}\right] \quad$ B XI kalendas

IX C X kalendas

D IX kalendas

(24 mai)

Commemoratio domini Wilhelmi Schevart (154) ac parentum, benefactorum, amicorum et omnium fidelium defunctorum, qui legavit nobis suum annum gratie ad emendum hereditates, cum I alio modio de quo hereditati sumus apud Oteey $\left({ }^{b}\right)\left({ }^{c}\right)(155)$.

$\left[25 r^{\circ}\right]$ XVII E VIII kalendas

(25 mai)

Urbani (156) pape et martyris. Collecta $\left({ }^{(}\right)$.

$\left({ }^{a}\right)$ D : écriture de type littera cursiva formata $\left(X I V^{e}-X V^{e}\right.$ s. $) .-\left({ }^{b}\right) E$ : écriture de type littera cursiva $\left(X V^{e}\right.$ s.). $-\left({ }^{c}\right)$ Sur le quart inférieur $d u f^{p} 24 v^{o}$, notula (indications comptables datant de l'époque moderne). - $\left(^{d}\right)$ et missa, suscrit.

(153) Jean (de) Banow devint chanoine de Saint-Materne le 6 février 1344. On le rencontre cité de 1344 au $1^{\text {er }}$ octobre 1360. Il était décédé le 30 avril 1374. Cf. LAHAYe, Saint-Materne, p. 109. - C.S.L., t. 4, pp. 114, 255, 290, 320, 328. - PonCelET, Sainte-Croix, t. 1, p. 208. - BerLlère, Suppliques Clément $V I, \mathrm{n}^{\circ} 660$, p. 152. - BERLIÈRE, Lettres Clément VI, $\mathrm{n}^{\circ} 577$, p. 187.

(154) Guillaume Schevart, chanoine (sous-diacre) de Saint-Materne dès le 19 décembre 1435 . Il quitta Liège le 26 juillet 1437 mais il revint car il est cité jusqu'en 1474. Cf. LahaYe, Saint-Materne, pp. 147-148. - Poncelet, Sainte-Croix, t. 2, p. 19.

(155) Othée. Cf. note 84.

(156) Saint Urbain I ${ }^{\text {er }}$ pape, martyr. Fête : le 25 mai. 
Commemoratio Egidii, sacerdotis concanonici nostri, pro quo habemus domum I prope molendinum in Toringio (157) quae reddit VIII solidos.

VI F VII kalendas

(26 mai)

Commemoratio magistri Amelii (158), quondam decani Sancti Dyonisii Leodiensis, pro quo habemus II modios spelte apud Vorous (159) iuxta Bierses (160).

G VI kalendas

(27 mai)

Commemoratio domini Herberti de Acquis (161) $\left({ }^{a}\right)$, canonici Leodiensis, pro quo habemus III modios et dimidium siliginis apud Glenes (162) super II bonuariis terre virgata minus.

$\left[25 v^{0}\right]$ XIIII A V kalendas

(28 mai)

Germani (163) episcopi et confessoris. Commemoratio.

${ }^{(a)}$ presbyteri, suscrit.

(157) Torrent (en), quartier de Liège situé entre la rue de l'Université et la rue de la Régence, d'une part ; entre la place de la République française et la place Cockerill, d'autre part. Cf. GoBerT, Les rues de Liège, t. 5, pp. 443-445.

(158) Maître Amel de Fize, cité comme collecteur apostolique dans le diocèse de Liège le 28 mars 1247, date à laquelle lui est réservé un bénéfice vacant. Il est cité comme chanoine de Saint-Materne, le 30 mai 1254, comme chanoine de Notre-Dame de Tongres, le 12 octobre 1258, et comme chanoine de la collégiale Saint-Denis de Liège du 3 août 1267 au 28 janvier 1268, comme official du prévôt Jean de Condé le 25 septembre 1271. Il est doyen de Saint-Denis dès 1272 (n. st.) et est cité jusqu'en 1283. Cf. RenaRDy, Les maîtres universitaires, pp. 171-172. Il est commémoré le 26 mai dans l'ob. N.-D. Tongres, éd. Paquay, B.S.S.L.L., t. 41, 1927, p. 82 et le 27 mai dans l'ob. Saint-Denis, fo $9 \mathrm{v}^{\circ}$.

(159) Voroux-Goreux (pr. Liège, arr. Waremme, comm. Fexhe-le-Haut-Clocher).

(160) Bierset (pr. Liège, arr. Liège, comm. Grâce-Hollogne).

(161) Herbert d'Aix, chanoine de Saint-Lambert de 1187 à 1242. Cf. Chot-Stassart, p. 24. - C.S.L., t. 1, pp. 109, 163, 184, 332, 356, 358, 392, 425. - PONCELET, Hugues de Pierrepont, pp. 16, 18, 162.

(162) Gelinden, commune de Gelmen (pr. Limbourg, arr. Hasselt, comm. Saint-Trond).

(163) Saint Germain, évêque, conf. Fête : le 28 mai. 
Commemoratio magistri Johannis de Psalmis (164) pro quo habemus $\mathrm{X}$ sextarios spelte apud Horpale (165).

III B IIII kalendas

Maximini (166) episcopi et confessoris. Commemoratio.

Commemoratio magistri Egidii de Canistrata (167) et Egidii, eius patris, pro quibus habemus XIII solidos bone in macello.

C III kalendas

(30 mai)

XI D II kalendas

Petronille (168) virginis. Collecta.

NOX HABET HORAS VIII, DIES XVI

(164) Maître Jean de Salm fut investi, dès le 17 janvier 1244, d'une prébende à Saint-Goar sur le Rhin (diocèse de Trèves) et de deux cures paroissiales. Il obtint ensuite un canonicat à Saint-Materne. Il est cité le 25 octobre 1271 comme propriétaire d'une maison située rue Haute-Sauvenière, à Liège. Cf. RENARDY, Les maîtres universitaires, pp. 370-371. - Schooniroodt, Val-Saint-Lambert, t. 1, p. 114.

(165) Horpmaal, commune de Heers (pr. Limbourg, arr. Tongres, comm. Heks).

(166) Saint Maximin, évêque, conf. Fête : le 29 mai.

(167) Maître Gilles de Chinstrée est cité le 6 août 1304 comme chanoine de la collégiale Sainte-Croix de Liège. Cf. RENARDY, Les maîtres universitaires, p. 248. - PonCELET, Sainte-Croix, t. 1, pp. CIII, 84-85. Il est commémoré le 29 mai dans l'ob. Sainte-Croix (I), $\mathrm{f}^{\circ} 132 \mathrm{v}^{\circ}$. - ob. Sainte-Croix (III), $\mathrm{f}^{\circ} 40 \mathrm{r}^{\circ}$.

(168) Sainte Pétronille, vierge non martyre. Fête : le 31 mai. 
$\left[26 r^{\circ}\right]$ JUNIUS IN DECIMO QUINDENUM A FINE SALUTAT (169)

\section{JUNIUS HABET DIES XXX, LUNA XXIX}

E kalendas

(1 juin)

Nichomedis (170) martyris. Collecta.

Commemoratio domini Hermanni de Zanctis (171), presbyteri canonici Leodiensis, pro quo habemus I modium spelte in granario nostro $\left({ }^{a}\right)$.

XIX F IIII nonis

(2 juin)

Marcellini (172) et Petri (173) martyrum. Collecta et missa.

VIII G III nonis

$\left[26 v^{c}\right]$ XVI A II nonis

(4 juin)

Commemoratio parentum domini Johannis de Banou (174), concanonici nostri, pro quibus habent quilibet XII denarios, presbyter XII denarios, et clericus VI denarios bone supra domum quondam ipsius in Torrengio (175) $\left(^{b}\right)$.

$\left({ }^{a}\right) D$ : écriture de type littera cursiva formata $\left(X I V^{e}-X V^{e}\right.$ s.). $-\left({ }^{b}\right) D$ : écriture de type littera cursiva formata $\left(X I V^{e}-X V^{e} s\right.$. $)$.

(169) La forme habituelle est Junius in deno quindenum a fine salutat. Cf. GroteFEND, Zeitrechnung, t. 1, p. 36. Cf. également, ici-même, note 1.

(170) Saint Nicomède, martyr. Dedicatio ecclesie : le $1^{\text {er }}$ juin.

(171) Cf. note 4.

(172) Saint Marcellin, martyr. Fête : le $1^{\mathrm{er}}$ juin.

(173) Saint Pierre, martyr. Fête: le 2 juin.

(174) Cf. note 153.

(175) Cf. note 157. 
Bonefacii (176) episcopi et martyris. Commemoratio.

Commemoratio $\left({ }^{a}\right)$ domini Johannis Borgondi (177), presbyteri concanonici nostri, pro quo habemus II marchas bone supra domum grandem Warneri $\left(^{b}\right)$.

C VIII idus

Commemoratio magistri Johannis dicti Mouthon (178), concanonici nostri, pro quo habemus II bonuaria terre apud Pepenges (179) solventia III modios siliginis.

\section{$\left[27 r^{\circ}\right]$ XIII D VII idus}

Commemoratio magistri Alvani (180) pro quo habemus III modios spelte in grenario nostro.

(a) Ce mot est interpolé. La fin de la commémoration sanctorale (com) a été employée comme début de la notice-obituaire. - $\left(^{b}\right) D$ : écriture de type littera cursiva formata $\left(X I V^{e}-X V^{e}\right.$ s.).

(176) Saint Boniface, évêque, martyr, et ses compagnons. Fête : le 5 juin.

(177) Cf. note 81.

(178) Maître Jean de Chimay ou Mouton, chanoine de Saint-Materne. En octobre 1231, il renonça à la chapellenie de Notre-Dame de Heigne (pr. Hainaut, arr. Charleroi, comm. Jumet). Il est cité jusqu'au 5 juillet 1270. Cf. ReNARDY, Les maîtres universitaires, pp. 336-337. - SCHOONBROODT, Val-Saint-Lambert, t. 1, p. 93. Il est commémoré le 7 juin dans l'O.S.L., éd. MarchandisSe, p. 78 et n. 484.

(179) Pepingen, commune de Mechelen-Bovelingen (pr. Limbourg, arr. Tongres, comm. Heers).

(180) Maître Alain de Liège, chanoine de Notre-Dame de Tongres et secrétaire de Jean d'Eppes (1236-1281), archidiacre de Brabant et coûtre de Saint-Lambert. Il était déjà décédé le 7 avril 1281 (n. st.). Ses volontés testamentaires sont en effet exprimées à cette date. Cf. Renardy, Les maitres universitaires, p. 169. - J. Ruwet, Cartulaire de l'abbaye cistercienne de Val-Dieu (XIT $-X I V^{e}$ siècles), Bruxelles, 1962, pp. 206-207. Ce maitre est également commémoré le 7 juin dans le Livre des anniversaires de l'abbaye du Val-Dieu (XIII ${ }^{t}$-XVII $T^{2}$ siècles), éd. J. RuwET, B.S.A.H.D.L., t. 36, 1950, p. 57, et le 8 juin dans l'ob. N.-D. Tongres, éd. PaquaY, B.S.S.L.L., t 41, 1927, p. 84. 
II E VI idus

(8 juin)

Medardi (181) episcopi et confessoris. Commemoratio.

Commemoratio domini Jacobi de Sancto Caro (182), quondam decani Sancte Marie Hoyensis $\left({ }^{a}\right)$, cum suo predecessore, pro quibus habemus II modios spelte apud Puchey $\left({ }^{b}\right)(183)$.

F V idus

(9 juin)

Primi et Felitiani (184) martyrum. Collecta et missa ${ }^{c}$ ).

$\left[27 v^{0}\right] \mathrm{X} \quad \mathrm{G} \quad$ IIII idus

(10 juin)

A III idus

(11 juin)

Barnabe (185) apostoli. IX lectiones.

Commemoratio domini Johannis de Sancta Maria (186), presbyteri canonici Parve Mense, pro quo habemus I modium spelte in granario nostro.

$\left({ }^{a}\right)$ Entre Hoyensis et cum, les mots pro quo habemus dimidium modium spelte, de la main $A$, sont biffés. $-\left({ }^{b}\right) D$ : écriture de type littera cursiva formata $\left(X I V^{e}-X V^{e} s.\right)$. - (') Au bas du $f^{\circ} 27 r^{\circ}$, notula (indications comptables datant de l'époque moderne).

(181) Saint Médard (Mard, Mars, Merd, Miard), évêque, conf. Fête : le 8 juin.

(182) Jacques le Cokins ou de Coquina, chanoine puis doyen de Notre-Dame de Huy, est cité le 10 avril 1318 et est commémoré le 11 juin dans l'ob. N.-D. Huy, éd. DeCKers-Renardy, p. 123. Cf. Génicot, Les chanoines de Huy, p. 68. - C.S.L., t. 2, p. 178. La proximité de la date de décès de l'ab. N.-D. Huy et de l'ob. Saint-Materne (le 8 juin) nous incline à penser que Jacques de Coquina et Jacques de Saint-Chair sont une même personne. Il convient néanmoins de mentionner l'existence d'un Jean de Saint-Chair, cité comme chanoine de Saint-Materne le 22 novembre 1343. Il est second en rang parmi les chanoines de Saint-Materne le $1^{\text {er }}$ octobre 1360 et premier le 30 avril 1374. Cf. LAHAYE, Saint-Materne, pp. 109, 121. - C.S.L., t. 4, pp. 21, 328, 508. Le prédécesseur de Jacques de Coquina est Émichon, cité le 16 février 1317. Cr. Génicot, Les chanoines de Huy, p. 68. - PonCelet, F.A.M., p. 185.

(183) Pousset (pr. Liège, arr. Waremme, comm. Remicourt) ou Poucet (pr. Liège, arr. Waremme, comm. Hannut).

(184) Saints Prime et Felicien, frères, martyrs. Fête : le 9 juin.

(185) Saint Barnabé, apôtre. Fête : le 11 juin.

(186) Un Jean de Notre-Dame, clerc de Liège, est témoin dans un acte daté du 30 juin 1330 . Cf. C.S.L., t. 3, p. 358. 
XVIII B II idus

(12 juin)

Basilidis (187) cum sociis suis martyrum. Collecta.

Commemoratio Beatricis, famule magistri Walteri, pro qua habemus dimidium modium spelte in granario nostro $\left({ }^{a}\right)$.

$\left[28 r^{\circ}\right]$ VII C idus

D XVIII kalendas

XV E XVII kalendas

Viti (188) martyris. Collecta.

Commemoratio domini Brononis de Boechem (189), canonici Leodiensis, qui legavit nobis partem domus sue claustralis de quo habemus primo super dimidietas $X X I X$ virgarum terre jacentium in territorio de Harstapel (190), II modios spelte; item in Odeur (191) II modios spelte, de quo habemus litteras, super terras quas tenet pro presenti Goffardus Thiertemps et tenet $\left(^{b}\right)$ mediantibus tres modii spelte et debet distribui istud aniversarium in promptu presentibus in vigiliis $\left({ }^{c}\right)\left({ }^{d}\right)$.

$\left({ }^{a}\right)$ Le $f^{o} 27 v^{o}$ comporte des taches. - ${ }^{b}$ ) Trois mots illisibles (taches). - ( $)$ C: écriture de type littera textualis $\left(X I V^{e}-X V^{e}\right.$ s.). - $\left({ }^{d}\right)$ Le $f^{\circ} 28 r^{\circ}$ comporte des taches. En bas de page, notula (indications comptables datant de l'époque moderne).

(187) Saints Basile, Cyrin, Nabor (Avold, Avon), Nazaire (Nazar, Anazar, Senari), martyrs. Fête : le 12 juin.

(188) Saint Vit (Wido, Guido), martyr avec saint Modeste et sainte Crescence. Fête : le 15 juin.

(189) Brunon de Broechem ou de Bruxhem était chanoine de Saint-Lambert en 1430. Il est cité en 1432 et en 1434 , à cette dernière date comme grand compteur du chapitre cathédral et comme chanoine de Saint-Lambert. Il était également prévôt de Saint-Rombaut de Malines. Il mourut le 13 juin 1456. Cf. DE Theux, S.L., t. 2, p. 216. - Poncelet, Sainte-Croix, t. 1, p. 503.

(190) Herstappe. Cf. note 75.

(191) Odeur (pr. Liège, arr. Waremme, comm. Crisnée). 
$\left[28 v^{o}\right] \quad$ IIII $\quad$ F XVI kalendas

(16 juin)

Commemoratio domini Lamberti de Stabulis (192), presbyteri, pro quo habemus $\mathrm{V}$ solidos bone quos debet ecclesia Sancte Crucis.

G XV kalendas

(17 juin)

Commemoratio Arnuldi (193), dyaconi, dicti predicatoris, pro quo habemus III modios spelte super terram quam emimus apud Marlines (194) prope Glins (195).

XII A XIIII kalendas

(18 juin)

Marci et Marcelliani (196) martyrum. Collecta et missa.

Gervasi et Prothasi (197) martyrum. Collecta $\left({ }^{a}\right)$.

$\left.{ }^{a}\right)$ et missa, suscrit.

(192) Lambert de Stavelot, chanoine de Rutten/Russon (pr. Limbourg, arr. Tongres, comm. Tongres), cité dès le 7 août 1313. Il mourut le 16 juin 1337. Divers actes relatifs à ses dispositions testamentaires sont conservés. Cf. C.S.L., t. 3, p. $285 ;$ t. 4 , p. $34 ;$ t. 6 , p. 51. - Poncelet, Sainte-Croix, t. 1, pp. CLXXIV, 150, 156, 178. Il est commémoré le 15 juin dans l'O.S.L., éd. MarChandisSe, p. 81 et n. 501 ; le 17 juin dans l'ob. Sainte-Croix (I), $\mathrm{f}^{\circ} 133 \mathrm{v}^{\circ}$. -- ob. Sainte-Croix (II), $\mathrm{f}^{\circ} 58 \mathrm{r}^{\circ}$. - ob. Sainte-Croix (III), $f^{\circ} 44 v^{\circ}$.

(193) Maître Arnould, prédicateur et chanoine de la Petite-Table, est cité de 1226 à août 1237. Il servit à de nombreuses reprises d'intermédiaire entre le pape et l'empereur. Cf. Renardy, Les maitres universitaires, p. 177. Il est commémoré le 17 juin dans l'O.S.L., éd. MarchandisSe, p. 84 et n. 513 et dans le Polyptyque de Saint-Lambert, p. 190.

(194) Marlines, Mechelen-Bovelingen, commune de Heers (pr. Limbourg, arr. Tongres, comm. Heks).

(195) Gelinden. Cf. note 162.

(196) Saints Marc et Marcellin, martyrs. Fête : le 18 juin.

(197) Saints Gervais et Protais, martyrs. Fête: le 19 juin. 
Commemoratio domini Johannis Labelle (198), presbyteri, pro quo habemus $\mathrm{V}$ solidos bone cum I capone in Sancto Christoforo (199).

\section{XII kalendas}

(20 juin)

Commemoratio domini Geleni de Sarto (200), decani Sancti Dyonisii, pro quo habemus apud Ohey(201) I

(198) Jean Label(le), chanoine de Saint-Materne, chapelain de Saint-Lambert, est cité du 14 septembre 1291 au 14 novembre 1314. Cf. C.S.L., t. 2, p. 492 ; t. 3, pp. 32, 61, 68, 117, 146. - Paquay, Cartulaire N.-D. Tongres, t. 1, pp. 246-247. - Bormans-SCHOOLMEESTERS, Liber officiorum, p. $482 \mathrm{n}$. 1. Jean Labelle est commémoré le 18 juin dans l'O.S.L., éd. Marchandisse, p. 84 et n. 519 ; le 19 juin dans l'ob. N.-D. Tongres, éd. PaQuay, B.S.S.L.L., t. 41, 1927, p. 86 ; le 23 juillet dans l'ob. Sainte-Croix (I), $\mathrm{f}^{\circ} 136 \mathrm{r}^{\mathrm{o}}$. - ob. Sainte-Croix (II), $\mathrm{f}^{\circ} 63 \mathrm{r}^{\circ}$. - ob. Sainte-Croix $(I I I), \mathrm{f}^{\circ} 52 \mathrm{v}^{\circ}$.

(199) Église Saint-Christophe à Liège. Cf. GoBERT, Les rues de Liège, t. 2, pp. 363-368.

(200) Ghislain de Sart, né en 1379, est mentionné comme chanoine de Saint-Materne en 1408. Il démissionna le 23 octobre 1410 en faveur de Gérard de Sart (cf. note 301). Il est cité le 10 mai 1419 comme maître ès-arts, diacre du diocèse de Liège, sous-collecteur de la Chambre apostolique à Liège, chanoine prébendé de la collégiale Saint-Denis de Liège (avant 1415) et de Notre-Dame d'Aix-la-Chapelle (dès 1411), chapelain à Florennes (pr. Namur, arr. et comm. Philippeville) et à Robermont (abbaye de Liège). Il est seigneur de Froidmont [dép. de Jemeppe-sur-Sambre (pr. Namur, arr. Namur)] du 21 février au 29 juin 1423. En 1424, il succéda à Gilles de Vinalmont ( $\left.{ }^{1424}\right)$ comme doyen de la collégiale Saint-Denis et est cité comme tel le 31 mars 1428, le 19 septembre 1432 , le 8 décembre 1433 , en 1436 et le $1^{\text {er }}$ juillet 1443 , lorsqu'il fut nommé collecteur de la Chambre pour le diocèse de Liège par le pape Eugène IV (1431-1447). Il est admis comme chanoine de la collégiale Saint-Pierre de Liège le 2 avrił 1429, est forain en 1431, est réadmis le 28 mai 1442, résigne le 26 juin 1444 et meurt, semble-t-il, le 16 juillet 1444 , sans avoir rendu ses comptes de collecteur. Ses biens seront donc séquestrés le 12 mai 1446. Il fut chancelier de l'évêque de Liège Jean de Heinsberg puis, en 1429, du duc de Brabant. Cf. A. DE GERADON, L'étrange carrière du chanoine Gilain de Sart (1379-1444), chancelier de Liège et de Brabant, B.I.A.L., t. 88, 1976, pp. 123-150 (donne comme date de décès : 16 juin) - F. BAIX, La chambre apostolique et les "libri annatarum " de Martin $V(1417-1431)$, t. 1, Introduction et textes, Analecta Vaticano-Belgica, t. 14, Bruxelles-Rome, 1947, pp. XLIV et n. 5, LXV et n. 4, LXVI, CI et n. 1, CLXXXI, CLXXXIV n. 1 et 4, CLXXXV n. 3, CCLXIX, n ${ }^{\text {os }} 41,63,297,511,1018$, pp. 12-13 et n. 7,20 n. 3,97 n. $5,189-190$ n. 6 (donne comme date de décès : 16 juillet 1444), 380 n. 1 . - C.S.L., t. 5, pp. 87, 101 ; t. 6, p. 180 - Bormans, Saint-Denis, p. 160 n. 1. - PonCELET, Saint-Pierre, pp. LVI, LXXXV. - LAHAYE, Saint-Materne, p. 143. - É. SCHOOLMEESTERS, Les doyens de la collégiale Saint-Denis, LeOdIUM, t. 9, 1910, pp. 95-96. - JEAN DE Stavelot, Cinquième chronique du pays de Liège, éd. A. Borgnet, Bruxelles, 1861, pp. 168, 496. Pour son épitaphe à la collégiale Saint-Denis, cf. Naveau-Poullet, t. 1, p. 127.

(201) Ohey (pr. Namur ; arr. Namur ; comm. Namur). 
modium spelte super I pratum existentem apud Blehen $\left({ }^{a}\right)(202)$.

IX D XI kalendas

(21 juin)

Albani (203) martyris. Commemoratio.

E X kalendas

(22 juin)

Paulini $\left({ }^{b}\right)(204)$ confessoris. Commemoratio.

Commemoratio domini Johannis de Jupilia (205), dyaconi canonici Leodiensis, pro quo habemus XIX modios spelte recipiendos de bonis nobis a dicto Johanne legatis in Villare (206) et in territorio de Miremorte (207) et de Votemme (208).

XVII F IX kalendas

(23 juin)

Vigilia.

Commemoratio domini Jacobi de Ugrees, presbyteri, pro quo habemus XII sextarios spelte.

Item, eodem die, commemoratio domini Henrici de

$\left(^{a}\right)$ E: écriture de type littera cursiva $\left(X V^{e}\right.$ s.). $-\left({ }^{b}\right)$ episcopi et, suscrit.

(202) Blehen (pr. Liège ; arr. Waremme ; comm. Hannut).

(203) Saint Aubain, martyr. Fête : le 21 juin.

(204) Saint Paulin, évêque, conf. Fête : le 22 juin.

(205) Jean de Jupille est cité comme chanoine de Saint-Lambert entre 1260 et 1293.

Cf. Chot-Stassart, p. 88. - Poncelet, Sainte-Croix, t. 1, p. 61 . Il est commémoré le 22 juin dans l'O.S.L., éd. Marchandisse, p. 87 et n. 534. - ob. Sainte-Croix (I), $\mathrm{f}^{\circ} 134 \mathrm{r}^{\circ}$. - ob. Sainte-Croix (II), $\mathrm{f}^{\circ} 59 \mathrm{r}^{\circ}$. - - ob. Sainte-Croix (III), $\mathrm{f}^{\circ} 45 \mathrm{v}^{\circ},-o b$. N.-D. Tongres, éd. PaquaY, B.S.S.L.L., t. 41, 1927, p. 86; le 24 juin dans l'ob. Saint-Denis, $f^{\circ} 10 \mathrm{v}^{\circ}$ et le 2 novembre dans l'ob. Saint-Barthélemy, $\mathrm{f}^{\circ} 39 \mathrm{v}^{\circ}$.

(206) Villers-l'Évêque. Cf. note 25.

(207) Milmort (pr. Liège, arr. Liège, comm. Herstal).

(208) Vottem. Cf. note 105. 
Alken (209), concanonici nostri, qui legavit nobis annum gratie et antiqua debita sua ad emendum hereditates pro anniversario suo binies in anno perpetue faciendo $\left({ }^{a}\right)$.

Commemoratio domini Henrici de Halken, concanonici nostri presbyteri, pro quo habemus apud Kemexhe (210), ut patet in registro nostro Sancti Materni, $X$ sextarios spelte $\left({ }^{b}\right)$.

VI G VIII kalendas

(24 juin)

Nativitas Sancti Johannis Baptiste (211). Totum duplex.

$\left[30 r^{\circ}\right]$

A VII kalendas

(25 juin)

Gallicani (212) martyris. Commemoratio.

XIIII B VI kalendas

(26 juin)

Johannis et Pauli (213) martyrum. III lectiones.

III C V kalendas

(27 juin)

Septem dormientium martyrum (214). Commemoratio.

$\left({ }^{a}\right) B:$ écriture de type littera textualis formata $\left(X V^{e} s\right) .-\left({ }^{b}\right) E: \dot{e c r i t u r e}$ de type littera cursiva $\left(X V^{e}\right.$ s.).

(209) Henri d'(H)Alken, chanoine (prêtre) de Saint-Materne, succède à Guillaume Stut $\left(\dagger 17\right.$ juin 1433), le 22 juin 1433 . Il est cité en 1436 , le $1^{\text {er }}$ mars 1437 , le 23 juin 1444 , le 11 août 1445 et le 25 avril 1447. Cf. LAHAYe, Saint-Materne, p. 111. - PonCelet, Sainte-Croix, t. 2, p. 7. - C.S.L., t. 5, pp. 111, 132, 135. - Cuvelier, Inventaire Val-Benoit, pp. 271-272.

(210) Kemexhe (pr. Liège, arr. Waremme, comm. Crisnée).

(211) Saint Jean-Baptiste, martyr. Sa nativité est fêtée le 24 juin.

(212) Saint Gallican, martyr. Fête : le 25 juin.

(213) Saints Jean et Paul, frères, martyrs. Fête : le 26 juin.

(214) Les sept Dormants (Maximianus, Malchus, Martinianus, Dionysius, Johannes, Serapio, Constantinus), martyrs. Fête : le 27 juillet. 
Leonis pape $\left({ }^{a}\right)(215)$. Collecta. Vigilia.

Commemoratio Arnuldi de Okirs (216), concanonici nostri, pro quo habemus IIII modios spelte recipiendos super II bonuariis terre jacentibus apud Vorous (217), acquisitis per dictum Arnuldum.

XI E III kalendas

Petri (218) et Pauli (219) apostolorum. Duplex.

F II kalendas

Celebratio $\left({ }^{b}\right)$ Pauli. IX lectiones.

NOX HABET HORAS VI, DIES XVIII

$\left({ }^{a}\right)$ et confessoris, suscrit. - $\left({ }^{b}\right)$ sancti, suscrit.

(215) Saint Léon II, pape. Fête : le 28 juin.

(216) Cf. note 21.

(217) Il s'agit soit de Voroux-lez-Liers (pr. Liège, arr. Liège, comm. Juprelle), soit de Voroux-Goreux (pr. Liège, arr. Waremme, comm. Fexhe-le-Haut-Clocher).

(218) Saint Pierre, apôtre, martyr. Fête principale : le 29 juin.

(219) Saint Paul, apôtre, martyr. Sa principale fête est célébrée avec celle de saint Pierre, le 29 juin. Sa commémoration a lieu le 30 juin. 


\title{
$\left[31 r^{\circ}\right]$ TREDECIMUS IULII DECIMO INNUIT ANTE KALENDAS
}

\author{
JULIUS HABET DIES XXXI, LUNA XXX
}

XIX G kalendas

(1 juillet)

Octava Sancti Johannis Baptiste (220). IX lectiones. Monegundis (221) virginis. Commemoratio.

Commemoratio domini Hermanni de Zanctis (222), presbyteri canonici Leodiensis, pro quo habemus I modium spelte supra granarium nostrum $\left({ }^{a}\right)$.

Eadem die, commemoratio domini Johannis Egidii (223), cardinalis et conprepositi Leodiensis, pro quo habemus II modios spelte et duobus eis anniversarius fieri in capella nostra iuxta conditiones anniversarii domini Anthonii de Moylant et debentur modii ad computatorem anniversa-

(a) D : écriture de type littera cursiva formata (XIVe-XVe s.). let.

(220) Octave de la nativité de saint Jean-Baptiste et sa circoncision. Fête : le $1^{\text {er }}$ juil-.

(221) Sainte Monégonde, vierge. Fête : le $1^{\text {cr }}$ juillet, à Liège.

(222) Cf. note 4.

(223) Jean Gilles, docteur en droits canon et civil, chanoine de Paris. Il fut reçu comme auditeur du palais apostolique le 12 décembre 1380 . Il acquit la prévôté de Saint-Lambert après mai 1381 et est cité comme prévôt du 12 février 1382 à sa mort, à Pise, le $1^{\text {er }}$ juillet 1408. Il fut plusieurs fois chargé de mission en France, en Allemagne, en Italie et en Bohême. À la curie, il fut référendaire, auditeur du palais apostolique et des lettres contradictoires ainsi que chapelain d'honneur. Il fut crée cardinal-diacre au titre des Saints-Cosme-et-Damien le 12 juin 1405 par le pape Innocent VII (1404-1406) et fut surnommé le "cardinal de Liège». Cf. Chot-STASSART, p. 156. - C.S.L., t. 4, pp. 626,646 ; t. 5 , pp. $28,30,45,51,55,58,59,567$; t. 6 , pp. $148,154,159,165$. - FAlRON, Regestes, t. 3, p. 2. - M. Gastout, Suppliques et lettres d'Urbain VI (1378-1389) et de Boniface IX (cinq premières années: 1389-1394). Analecta Vaticano-BelgiCa,

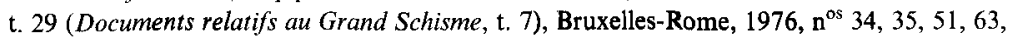
94, 98, 519, pp. 37-38, 51, 57, 86, 89, 459 n. 193. - Chr. DuRY, Les curialistes belges à Rome et l'histoire de la curie romaine, problème d'histoire de l'Église. L'exemple de Saint-Lambert à Liège, B.I.H.B.R., t. 50, 1980, pp. 150, 156 n. 39 . Il est commémoré le 5 juillet dans l'obituaire de l'église collégiale, libre et impériale de Saint-Servais à Maestricht, commencé vers 1294, éd. DoppleR, P.S.H.A.L., t. 47, 1911, p. 324 (selon lui, Jean Gilles est décédé en juillet 1407 ; selon Снот-STASSART, p. 156, en 1410). 
riorum dominorum et debent distribui in promptu inter presentes $\left(^{(}\right)$.

VIII A VI nonis

(2 juillet)

Processi et Martiniani (224) martyrum. Collecta et missa. Visitatio Beate Marie $\left(^{b}\right)(225)$.

B V nonis

( 3 juillet)

Translatio Sancti Thome (226) apostoli. Collecta.

Commemoratio Henrici de Haloes (227), canonici Leodiensis, pro quo habemus IIII modios spelte apud Wamon (228).

$\left[31 v^{\circ}\right]$ XVI C IIII nonis

(4 juillet)

Translatio Sancti Martini (229) episcopi et confessoris. III lectiones. Ulrici (230) episcopi et confessoris. Commemoratio.

Commemoratio domini Wilhelmi de Richel (231), concanonici nostri, pro quo habemus II modios cum III sextariis spelte $\left({ }^{c}\right)$.

$\left({ }^{a}\right)$ E : écriture de type littera cursiva $\left(X V^{e}\right.$ s.). $-\left({ }^{b}\right)$ D : écriture de type littera cursiva formata $\left(X I V^{e}-X V^{e} s\right.$.). - ( $\left.{ }^{(}\right) C$ : écriture de type littera textualis $\left(X V^{e} s.\right)$.

(224) Saints Processe et Martinien, martyrs. Fête : le 2 juillet.

(225) Visitation de la Vierge à sainte Élisabeth. Fête : le 2 juillet.

(226) Saint Thomas, apôtre. Fête de sa translation : le 3 juillet.

(227) Henri de Hallois, chanoine de Saint-Lambert, est cité de 1249 à 1283. Il était décédé le 15 mai 1294. Cf. Chot-Stassart, p. 80. Il est commémoré le 26 novembre dans l'O.S.L., éd. MaRCHANDISSE, p. 162 et n. 1036.

(228) Wamont/Waasmont. Cf. note 12.

(229) Saint Martin, évêque, conf. La fête de son ordination et de sa translation est célébrée le 4 juillet.

(230) Saint Udalric (Ulric, Oury), évêque, conf. Fête : le 4 juillet.

(231) Guillaume de Ryckel ou de Richelle, chanoine (prêtre) de Saint-Materne, remplaça Renier de Vilhe (cf. note 99), qui était décédé en 1383. Il est cité le 17 juin 1392 et le 20 juillet 1394. Il mourut le 3 juillet 1397. Cf. Lahaye, Saint-Materne, p. 117 . C.S.L., t. 5, pp. 6, 12 . 
Commemoratio domini Johannis de Prato (232), canonici Sancti Servatii Traiectensis dudum concanonici nostri, pro quo habemus III solidos et IIII denarios bone et sextam partem IIII caponum supra domum Egidii Ficon en Bermonbech (233). Inde redduntur domino Petro le Borgegnon $\left({ }^{a}\right)$ VI denarii bone. In Nativitate Domini $\left({ }^{b}\right)$, pro $\left({ }^{c}\right)$ altaris $\left({ }^{d}\right)$. In ecclesia Beati Bartholomei. Sancti Nicholai $\left({ }^{(}\right)$.

E II nonis

(6 juillet)

Octava Petri et Pauli (234) apostolorum. IX lectiones. Goaris (235) confessoris. Commemoratio.

$\left(^{a}\right)$ Les mots domino Petro le Borgegnon ont été biffés puis restitués. - ${ }^{b}\left({ }^{b}\right)$ Le mot creatori est suscrit, d'une autre main $(B) .-\left({ }^{c}\right)$ Le mot est gratté. - ${ }^{(d)}$ Les deux derniers mots, Sancti Nicholai, se rapportent peut-être à cet autel. $-\left({ }^{e}\right)$ Ces deux mots ont été rédigés par une main postérieure $(B)$.

(232) Jean de Preit, chanoine de Saint-Materne, est cité le 24 décembre 1318 et le 26 août 1333. Il était décédé le $1^{\mathrm{er}}$ octobre 1360. Cf. LAHAYE, Saint-Materne, p. 109. HemricourT, t. 2, p. 469 . - C.S.L., t. 3, pp. 187, 423; t. 4, p. 328.

(233) Gilles dit Fichon, en Bermonbèche, clerc d'origine liégeoise. Le 10 juillet 1361, il sollicita une prébende à la collégiale Saint-Jean l'Évangéliste de Liège. Le 6 mai 1362, il est chapelain à la collégiale Saint-Denis de Liège, puis est désigné, le 11 décembre, comme chapelain perpétuel de Saint-Denis et scripteur papal. Le 13 février 1363, il résigna sa prébende à la collégiale Saint-Pierre, qu'il avait acquise en 1362, et devint chanoine de Saint-Denis, te 10 mai, charge qu'il résigna la même année pour une prébende à la Cathédrale, reçue le 25 novembre 1363. Il est nommé abbé séculier de Maaseik (pr. Limbourg, arr. et comm. Maaseik) le 26 mars 1364 et est cite soit avec ce titre, soit avec celui de chanoine de Saint-Lambert jusqu'en 1408. Cf. Chot-STassarT, p. 125. - C.S.L., t. 4 , pp. $578,600,602,614,652$; t. 6 , pp. 131-141. - Cuvilier, Val-Benoit , p. 672 . VAN Der MAde, Guillemins, pp. 77-79, 81, - Fierens, Letires Urbain V, t. 1, $\mathrm{n}^{\text {os }} 446$, $614,776,841,998,1075,1095,1545,1546$, pp. 152, 220, 321, 354, 423, 456, 466, 696-697. - Fierens, Suppliques Urbain V, $\mathrm{n}^{\mathrm{os}} 2$, 454, 619, 909, 1148, 1230, 1240, pp. 3-4, 133, 192 , 307-308, 410, 450-451, 456. - BERLIÈRE, Suppliques Innocent VI, n ${ }^{\circ} 1720$, p. 699 et n. 1. - Tinon, Lettres Urbain V, t. $2, \mathrm{n}^{\circ} 2397$, p. 244, n. 1. - TiHoN, Lettres Grégoire XI, t. 3, n. 3052 , p. 30 et n. 2. - B. Guillemain, La cour pontificale d'Avignon (1309-1376). Étude d'une société, Paris, 1962, p. 376 n. 130. - Chr. Dury, Les curialistes belges à Rome et l'histoire de la curie romaine, problème d'histoire de l'Église. L'exemple de Saint-Lambert à Liège, B.I.H.B.R., t. 50, 1980, pp. 149 et n. 5, 155 n. 5.

(234) Cf. note 219. L'octave est célébrée le 6 juillet.

(235) Saint Goar, conf. Fête : le 6 juillet. 
II G VIII idus

( 8 juillet)

Kyliani (236) cum sociis suis martyrum. Commemoratio.

A VII idus

(9 juillet)

Commemoratio domini Hermanni de Blehen (237). Octava visitationis Beate Marie ${ }^{a}$ ).

B VI idus

(10 juillet)

Septem fratrum martyrum (238). Collecta.

C V idus

(11 juillet)

Translatio Sancti Benedicti (239) abbatis et confessoris. Collecta.

XVIII D IIII idus

(12 juillet)

Cleti (240) pape et martyris. Collecta.

$\left({ }^{a}\right)$ E : écriture de type littera cursiva $\left(X V^{e}\right.$ s. $)$.

(236) Saint Kilien (Killanus, Chilianus, Quellien, Kilian) et ses compagnons (Col(o)mannus, (T)Otmannus ou Totnanus), martyrs. Fête : le 8 juillet.

(237) Herman de Blehen, chanoine (diacre) de Saint-Materne, remplaça Jean de Bréda $(\dagger 1399)$ et ne siégea que quelques années. Il est cité le 10 décembre 1401. Cf. LAHAYE, Saint-Materne, pp. 135-136. - C.S.L., t. 6, p. 158.

(238) Sept frères martyrs (Januarius, Felix, Philippus, Silvanus, Alexander, Vital, Martial), fils de Félicité. Fête : le 10 juillet.

(239) Translation de saint Benoît : le 11 juillet.

(240) Saint Clet (Anaclet), pape, martyr. Fête : le 13 juillet, à Liège. 
$\left[33 r^{0}\right] \quad \operatorname{VII}\left({ }^{a}\right) \quad$ E $\quad$ III idus

Margarete (241) virginis. III lectiones.

Commemoratio domini Balduini de Tectis (242) qui legavit nobis I modium spelte hereditarie supra domum suam sitam Leodii ultra Sanctum Paulum (243) in longo vico $\left({ }^{b}\right)$.

F II idus

(14 juillet)

Commemoratio domini Andree Pinte, capellani in ecclesia Leodiensis et quondam computatoris nostri, pro quo habemus $\mathrm{V}$ sextarios spelte.

Eodem die, commemoratio domini Johannis de Enzivaus (244), presbyteri canonici nostri, pro quo habemus $I X$ sextarios spelte apud Fexhe (245), de XX sextariis emptis a Johanne delle Soxhe (246). Item XII sextarios spelte in territorio de Lexi ( $\left.{ }^{c}\right)(247)$.

XV $\quad G$ idus

(15 juillet)

Divisio apostolorum (248). IX lectiones.

$\left(^{a}\right)$ La marge supérieure du folio $33 r^{\circ}$ comporte des fioritures ou des essais de plume. $-\left(^{b}\right)$ E : écriture de type littera cursiva $\left(X V^{e} s.\right) .-\left(^{c}\right) B$ : écriture de type littera textualis formata $\left(X I V^{e}-X V^{p}\right.$ s.).

(241) Sainte Marguerite, vierge (martyre). Fête : lc 13 juillet.

(242) Baudouin de Theux fut reçu chanoine-prêtre de Saint-Materne le 24 mars 1444. Son testament fut déposé au chapitre le 17 mai 1477 . Il avait résigné son canonicat car Nicolas Fabri, son successeur, émarge aux comptes de 1476-1477 au prorata de six mois. Cf. LAHAYE, Saint-Materne, p. 122.

(243) Collégiale Saint-Paul de Liège, actuelle cathédrale de Liège.

(244) Cf. note 125.

(245) Fexhe-Slins. Cf. note 37.

(246) Cf. note 37.

(247) Lexhy, dépendance de Horion-Hozémont (pr. Liège, arr. Liège, comm. GrâceHollogne).

(248) Divisio apostolorum. Fête: le 15 juillet. 
A XVII kalendas augusti

(16 juillet)

Gondulphi (249) et Monulphi (250) episcoporum et confessorum. Collecta.

B XVI kalendas

(17 juillet)

Fredegandi (251) et Alexis (252) confessoris. Commemoratio.

Commemoratio domini Godescalci de Dompire (253), canonici Leodiensis, pro quo habemus XVIII solidos et $\mathrm{V}$ capones apud Vinetum (254).

XII C XV kalendas

(18 juillet)

$\left[34 r^{\circ}\right] \quad I$

D XIIII kalendas

(19 juillet)

E XIII kalendas

(20 juillet)

IX F XII kalendas

(21 juillet)

Praxedis (255) virginis. Collecta $\left({ }^{a}\right)$.

Commemoratio domini Balduini de Trimeies, presbyteri, pro quo habemus XIII solidos et IIII denarios Leodienses.

$\left({ }^{a}\right)$ et missa, suscrit.

(249) Saint Gondon, évêque, conf. Fête : le 16 juillet, à Liège.

(250) Saint Monulphe, évêque, conf. Fête : le 16 juillet, à Liège.

(251) Saint Frédégand, évêque (abb.), conf. Fête : le 17 juillet, à Liège.

(252) Saint Alexis, conf. Fête : le 17 juillet.

(253) Godescalc de Dompierre, cité avec le titre de chanoine de Saint-Lambert de 1220 à 1238 . Il est peut-être déjà mentionné sous son seul prénom en 1209 et en 1214 . Il fut prévôt de la collégiale Saint-Barthélemy de Liège de 1233 à 1238. Cf. CHot-STASSART, p. 70. - De TheuX, S.L., t. 1, p. 232. - Paquay, Saint-Barthélemy, p. 74. Il est commémoré le 17 juillet dans l'O.S.L., éd. Marchandisse, p. 98 et n. 604 et dans le Polyptyque de Saint-Lambert, p. 191.

(254) Vivegnis. Cf. note 109.

(255) Sainte Praxède, vierge. Fête : le 21 juillet. 
Marie Magdalene (256). Duplex.

XVII A X kalendas

Appolinaris (257) episcopi et martyris. Collecta et missa.

VI B IX kalendas

(24 juillet)

Cristine (258) virginis. Commemoratio. Vigilia.

$[\cdots]$

$\left[35 r^{\circ}\right] \mathrm{XV} \quad \mathrm{A}$ idus

(13 août)

Ypoliti (259), cum sociis suis, martyrum. III lectiones.

Commemoratio domini Johannis de Bruges (260), presbyteri, pro quo habemus III modios spelte cum dimidio supra I bonuarium terre situm in territorio de Harstaple (261) in duabus petiis.

IIII B XIX kalendas septembris

Eusebii (262) confessoris. Collecta. Vigilia.

(256) Sainte Marie-Madeleine. Fête : le 22 juillet.

(257) Saint Apollinaire, évêque, martyr. Fête : le 23 juillet.

(258) Sainte Christine, vierge (martyre). Fête : le 24 juillet.

(259) Saint Hyppolite, martyr. Fête : le 13 août.

(260) Jean de Bruges, chanoine de Saint-Materne, est cité le 25 mai 1304. Cf. BERLİ̀RE, Instrumenta Miscellanea, $\mathrm{n}^{\circ} 8, \mathrm{p} .10$.

(261) Herstappe. Cf. note 75.

(262) Saint Eusèbe, presbyter, conf. Fête : le 14 août. 
C XVIII kalendas

Assumptio Beate Marie virginis. Totum duplex.

$\left[35 v^{\circ}\right]$ XII D XVII kalendas

(16 août)

Arnulphi (263) episcopi et confessoris. Collecta.

Commemoratio domini Pontii (264), canonici Leodiensis et presbyteri ac dudum concanonici nostri, pro quo habemus I modium spelte $\left({ }^{a}\right)$.

Octava Sancti Laurentii (265) martyris. III lectiones.

Commemoratio domini Johannis dicti Pexhon (266), canonici Sancti Petri Leodiensis et presbyteri ac dudum concanonici nostri, pro quo habemus II modios spelte $\left({ }^{\alpha}\right)$.

F XV kalendas

Agapiti (267) martyris. Collecta et missa.

$\left.{ }^{a}\right) B$ : écriture de type littera textualis formata $\left(X I V^{e}-X V^{e} s.\right)$.

(263) Saint Arnoul, évêque, conf. Fête : le 16 août.

(264) Il s'agit peut-être de Ponce Textor, cité comme chanoine de Liège de 1318 à 1325, date à laquelle il est mentionné comme coûtre de la collégiale Saint-Pierre de Liège, comme recteur de l'église paroissiale de Nézignan (France, dép. Herault) et comme archidiacre de l'église de Lizieux (France, dép. Calvados, ch.-1. d'arr.). Cf. Chot-Stassart, pp. 213-214. - FAIRON, Regestes, t. 1, pp. 524-526.

(265) Saint Laurent, archidiacre, martyr. Fête : le 10 août. Octave : le 17 août.

(266) Jean Pexhon est cité comme chanoine de Saint-Materne le $1^{\text {er }}$ avril 1343, le 4 février et le 3 mars 1355 ; comme chanoine de la collégiale Saint-Pierre de Liège, le 6 juillet 1357 et le 4 novembre 1376, date à laquelle il était décédé. Cf. Poncelet, Saint-Pierre, pp. 43, 58. - VAN Der MADE, Guillemins, p. 43. - FaIron, Regestes, t. 1, p. 397. - Poncelet, Sainte-Croix, t. 1, p. 308.

(267) Saint Agapet, martyr. Fête : le 18 août. 
$\left[36 r^{\circ}\right] \quad$ IX $\quad$ G XIIII kalendas

Magni (268) martyris. Collecta.

A XIII kalendas

Bernardi (269) abbatis ( ${ }^{a}$ ). III lectiones. Philiberti (270) abbatis et confessoris. Collecta.

Commemóratio Egidii de Daules (271), subdyaconi concanonici nostri, pro quo habemus I modium spelte supra domum domini H. Passet (272). Item XIII solidos bone supra domum ipsius Henrici ante ecclesiam Beate Magdalene (273) supra Legiam $\left({ }^{b}\right)$.

XVII B XII kalendas

(21 août)

Privati (274) episcopi et martyris. Commemoratio.

$<$ Commemoratio $>\left({ }^{(}\right)$domini Jasperis de Moreal$\operatorname{mes}\left(^{d}\right)(275)$.

$\left({ }^{a}\right)$ et confessoris, suscrit. $-\left({ }^{b}\right) C$ : écriture de type littera textualis $\left(X I V^{e}-X V^{e} s.\right)$. (c) Le mot est interpolé. La fin de la commémoration sanctorale (com) a été employée comme début de la notice-obituaire. $-\left({ }^{d}\right) E$ : écriture de type littera cursiva (fin $X V^{c} s$.).

(268) Saint Magnus, martyr. Fête : le 19 août.

(269) Saint Bernard de Clairvaux. Fête : le 20 août.

(270) Saint Philibert, abbé, conf. Fêtẹ : le 20 août.

(271) Gilles de Daules est cité comme chanoine de Saint-Materne le $1^{\text {er }}$ octobre 1360. Cf. LaHAYe, Saint-Materne, p. 109. - C.S.L., t. 4, p. 328.

(272) Henri Passet, chapelain de Saint-Lambert, est cité du $1^{\text {er }}$ octobre 1360 au 8 novembre 1372. Il était probablement décédé le 21 septembre 1390 . Cf. C.S.L., t. 3, p. 527 n. 1 ; t. 4 , pp. 329,497 ; t. 5 , p. 2.

(273) Église Sainte-Marie-Madeleine à Liège. Cf. LaHaye, Paroisses, p. 14 et passim.

(274) Saint Privat, évêque, martyr. Fête : le 21 août.

(275) Jaspar de Morialmé, chanoine (prêtre) de Saint-Materne, commence à émarger en avril 1471. Il est cité le 21 juin 1480 , le 26 janvier 1481 et le 29 septembre 1482 . Il testa le 22 août 1483. Cr. LAHAYE, Saint-Materne, p. 111. - C.S.L., t. 5, pp. 209, 211, 580. 


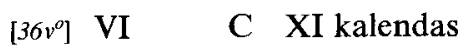

(22 août)

Octava Beate Marie. Duplex. Tymothei et Simphoriani (276) martyrum. Collecta.

Commemoratio domini Wilhelmi de Heers (277), presbyteri concanonici nostri, pro quo habemus apud Fyes (278) super III jornalia terre arabilis ut patet in cartulario Iwani inferius scripto, I modium spelte cum $V$ sextariis. Item habemus pro eodem. Item habemus IIII sextarios spelte apud Woroz ut patet in litera $\left({ }^{a}\right)$ de XX sextariis.

Anno Domini XIIII LXXX septimo, mensis augusti, die $X X I I$, obiit venerabilis vir dominus Johannes Lathomi (279), presbyter quondam canonicus Parve Mense, qui legavit nobis I calice argenteum cum suis pertinenciis et corporale ac custodiam eiusdem necnon unum missale papireum manualiter scriptum. Cuiusquidem calicis pondus est unius marche trium unciarum et decem esterlingorum $\left(^{b}\right)\left({ }^{c}\right)$.

D X kalendas

(23 août)

Timothei et Appollinaris (280) martyris. Collecta. Vigilia.

$\left({ }^{a}\right)$ Un mot illisible. $-\left({ }^{b}\right)$ Un mot illisible. $-\left({ }^{c}\right) E$ : écriture de type littera cursiva $\left(X V^{e}\right.$-début $X V F^{e}$ s.).

(276) Saints Timothée et Simphorien, martyrs. Fête : le 22 août.

(277) Guillaume de Heers, chanoine (sous-diacre) de Saint-Materne, est cité le 4 mai 1412. Il mourut en 1440. Cf. Lahaye, Saint-Materne, p. 139. - C.S.L., t. 6, p. 163.

(278) Il s'agit probablement de Fize-le-Marsal. Cf. note 113.

(279) Jean Lathomé (Lathomi) est admis comme chanoine de la collégiale Sainte-Croix de Liège, le 17 janvier 1453, mais résigna cette charge par échange en août 1457. Par son testament, daté du 20 août 1488, Jean Lathomé, chanoine de la Petite-Tabie, lègue un calice d'argent et un missel manuscrit aux chanoines de Saint-Materne. Il lègue sa maison en Basse-Sauvenière pour partie à son neveu Gérard Coppin, lequel fit don de cette maison aux chanoines de Saint-Materne par testament, approuvé en 1506. Jean Lathomé, qui fonda également son anniversaire aux Guillemins, est cité jusqu'au 5 mai 1496. Il était décédé le 21 juin 1500, date à laquelle est mentionné un autre testament de ce chanoine, daté du 15 juin 1500. Cf. LAHAYE, Saint-Materne, p. 125. - Poncelet, Sainte-Croix, t. 1, p. CXXXVII. - C.S.L., t. 5, p. 227. - VAN DeR MADE, Guillemins, pp. 164, 170-171, 174. - Bormans, Conclusions capilulaires, p. 63 . BORMANS-SCHOOLMEESTERS, Liber officiorum, p. 457.

(280) Saints Timothée et Apollinaire, martyrs. Fête : le 23 août. 
XIIII E IX kalendas

Bartholomei (281) apostoli. Duplex.

Commemoratio Hermanni, militis de Holongnele, pro quo habemus I modium cum dimidium siliginis et I caponem super XVI virgatas terre site apud Adyncurt prope Glendis $\left(^{a}\right)(282)$.

[37r" III $\quad$ F VIII kalendas

(25 août)

Genesii (283) martyris. Commemoratio.

G VII kalendas

(26 août)

Herenei et Habundi (284) martyrum. Commemoratio.

Commemoratio $\left({ }^{b}\right)$ domini Yvani Hebscaep (285) de Turnout, dyaconi concanonici nostri, pro quo habemus $I$ modium spelte apud Fyez (286) super III jornalia, de quibus habemus litteras.

Item commemoratio Henrici de Tegelen (287), quondam computatoris nostri, pro quo habemus supra easdem terras II sextarios et $\left({ }^{c}\right)$ II tertiarias spelte $\left({ }^{c}\right)$.

$\left({ }^{a}\right)$ Dans le bas du folio $36 v^{\circ}$, notula (indications comptables datant de l'époque moderne). - $\left({ }^{b}\right)$ Le mot est interpolé. La fin de la commémoration sanctorale (com) a été employée comme début de la notice-obituaire. - ( ) Le mot unum est biffé. - $\left.{ }^{d}{ }^{d}\right)$ E : écriture de type littera cursiva ( $X V^{e}$ s.).

(281) Saint Barthẻlemy, apôtre. Fête : le 24 août.

(282) Gelinden. Cf. note 162.

(283) Saint Genès, martyr. Fête : le 25 août.

(284) Saints Ireneus (Hereneus, Hireneus) et Abundus (Habundus), martyrs. Fête : le 26 août.

(285) Yvan Haebscap, chanoine (sous-diacre) de Saint-Materne, occupa cette charge de 1416 à sa mort, le 25 août 1439. Cf. LaHaye, Saint-Materne, p. 143.

(286) $\mathrm{Cf}$. note 113.

(287) Le 6 novembre 1432, une maison de la rue Pont d'Avroy est occupée par Henri de Teghelen et est chargée d'une redevance à payer au prieur et au couvent des Bons-Enfants à Liège. Cf. L. HALKIN, La maison des Bons-Enfants de Liège, B.I.A.L., t. 64, 1940, pp. 33-34. Un Henricus de Teghelen, civis Leodiensis, décédé le 6 octobre 1439, était inhumé au couvent des Croisiers de Liège. Cf. Naveau-Poullet, t. 1, p. 335. 
XI A VI kalendas

(27 août)

Rufi (288) martyris. Collecta.

Augustini (289) episcopi et confessoris $\left(^{a}\right)$. Hermetis (290) martyris. Collecta.

Commemoratio domini Nicholai Fabri (291), confratris nostri, pro quo habemus III marchas bone $\left({ }^{b}\right)$.

C IIII kalendas

(29 août)

Decollatio Sancti Johannis Baptiste $\left({ }^{c}\right)(292)$. Sabine (293) virginis. Collecta.

VIII D III kalendas

(30 août)

Felicis et Adaucti (294) martyrum. Collecta et missa.

E II kalendas

(31 août)

Paulini (295) episcopi et martyris. Commemoratio.

$\left({ }^{a}\right)$ duplex, suscrit. $-\left({ }^{b}\right)$ D : écriture de type littera cursiva formata $\left(X V^{e}\right.$ s.). (c) duplex, suscrit.

(288) Saint Ruf, martyr (évêque). Fête : le 27 août.

(289) Saint Augustin, évêque, conf. Fête : le 28 août.

(290) Saint Hermès, martyr. Fête : le 28 août.

(291) Nicolas Fabri succéda à Baudouin de Theux comme chanoine-prêtre de Saint-Materne. Il émargea aux comptes de 1476-1477 au prorata de six mois. Cf. LAHAY,, Saint-Materne, p. 122.

(292) Décollation de saint Jean-Baptiste (S.J. Décolasse, la Décolasse). Fête : le 29 août.

(293) Sainte Sabine, vierge (martyre). Fête : le 29 août.

(294) Saints Félix et Adaucte (Auctus, Audactus), martyrs. Fête : le 30 août.

(295) Saint Paulin, évêque, martyr. Fête : le 31 août. 
Commemoratio domini Florentii de Dyonanto (296), concanonici nostri et presbyteri, pro quo habemus II modios spelte.

NOX HABET HORAS X, DIES XIIII

\section{SEPTEMBER HABET DIES XXX, LUNA XXIX}

XVI $\quad F$ kalendas

(1 septembre)

Egidii (297) abbatis $\left({ }^{a}\right)$. IX lectiones. Prisci (298) martyris. Collecta.

Commemoratio magistri Walteri de Morseilh (299), concanonici nostri et presbyteri, pro quo habemus III modios spelte recipiendos in granario nostro.

Commemoratio domini Hermanni de Zanctis (300), presbyteri canonici Leodiensis, pro quo habemus I modium IIII sextarios spelte in granario nostro $\left(^{b}\right)$.

$\left({ }^{a}\right)$ et confessoris, suscrit. - $\left(^{b}\right) D:$ écriture de type littera cursiva formata $\left(X I V^{e}-X V^{e}\right.$ s.).

(296) Florent de Dinant, chanoine de Saint-Materne, est cité le 26 août 1333 et le 26 mars 1343. Il était décédé le $1^{\text {er }}$ octobre 1360 . Cf. LaHAYE, Saint-Materne, p. 109 . C.S.L., t. 3, pp. 423,579 ; t. 4, pp. 4, 328. RENARDY, Les maitres universitaires, p. 218 , propose d'identifier Florent de Dinant à maître Florent, chanoine de Sainte-Croix, cité le 14 février 1348. Remarquons qu'il ne porte pas le titre de magister dans sa commémoration.

(297) Saint Gilles, abbé (conf.). Fête : le $1^{\text {er }}$ septembre.

(298) Saint Priscus, martyr. Fête : le $1^{\text {er }}$ septembre.

(299) Cf. note 3.

(300) Cf. note 4. 
V G IIII nonis

(2 septembre)

Commemoratio Gerardi de Sarto (301), concanonici nostri. I modius spelte in granario $\left({ }^{a}\right)$.

A III nonis

(3 septembre)

Remacli (302) episcopi et confessoris. IX lectiones.

$\left[38 v^{\circ}\right]$ XIII B II nonis

(4 septembre)

Commemoratio Johannis de Hemricurt (303), concanonici nostri, pro quo habemus II modios spelte et dimidium in granario nostro.

II

C nonis

(5 septembre)

Commemoratio domini Johannis de Nazareyo, concanonici $\left({ }^{b}\right)$ nostri, pro quo habemus dimidium modium spelte recipiendum supra granarium nostrum.

\section{VIII idus}

(6 septembre) $\left({ }^{c}\right)$

$\left({ }^{a}\right)$ E : écriture de type littera cursiva $\left(X V^{e} s.\right) .-\left({ }^{b}\right)$ et presbyteri, suscrit. $-\left({ }^{c}\right)$ En bas $d u f^{\circ} 38 v^{\circ}$, notula (indications comptables datant de l'époque moderne).

(301) Frère probable de Ghislain de Sart (cf. note 200), Gérard de Sart, chanoine (sous-diacre) de Saint-Materne, fut reçu en 1410 et ne siégea que quelques années. Il est, en effet, cité le 21 juin 1412 et le 27 mai 1413. Il devint chanoine de la collégiale Saint-Barthélemy de Liège en 1432 et chanoine de Saint-Lambert le 29 mai 1445. Un Gérard de Sart (ou de Sars) est cité comme prêtre dès 1378 , le 8 mars 1401 et comme chapelain de Sainte-Croix le 14 mars 1405. C'est l'oncle du chanoine de Saint-Lambert. Cf. A. DE GERADON, L'étrange carrière du chanoine Gilain de Sart (1379-1444), chancelier de Liège et de Brabant, B.I.A.L., t. 88, 1976, pp. 123-150. PonCelet, Sainte-Croix, t. 1, pp. 427, 430. - PonCelet, Saint-Pierre, pp. 124, 141. - LAHAYE, Saint-Materne, p. 143.

(302) Saint Remacle, évêque, conf. Fête : le 3 septembre.

(303) Il est assez difficile d'identifier ce personnage qui appartient à la couche initiale, antérieure à 1372. Le plus vraisemblable est Jean-Thomas de Hemricourt, qui obtint une expectative de prébende à la collégiale Saint-Denis de Liège le 23 avril 1321 et est cité le 30 juin 1352. Cf. Hemricourt, t. 1, pp. 287, 291 ; t. 2, p. 246. - FAYEN, Lettres Jean $X X I I, \mathrm{t} .1, \mathrm{n}^{\circ}$ 912. p. 361 . 
Marci (304) pape et confessoris. Marcelli et Apulei (305) martyrum. Collecta et missa.

XVIII A VIII idus

(8 octobre)

Amoris (306) confessoris. Commemoratio.

VII B VII idus

(9 octobre)

Dyonisii (307), cum sociis suis, martyrum. Duplex.

$\left[39 v^{\circ}\right]$

C VI idus

(10 octobre)

Gereonis (308), cum sociis suis, martyrum. III lectiones.

Commemoratio Johannis de Longovico (309), dyaconi et concanonici nostri, pro quo habemus dimidium modium spelte supra granarium nostrum.

XV D V idus

(11 octobre)

IIII E IIII idus

(12 octobre)

(304) Saint Marc, pape, conf., martyr. Fête : le 7 octobre.

(305) Saints Marcel ct Apulée, martyrs. Fête : le 7 octobre.

(306) Saint Amour, diacre, conf. Fête : le 8 octobre, à Liège.

(307) Saint Denis, évêque et martyr. Fête, en compagnie de Rustique et Éleuthère : le 9 octobre.

(308) Saint Géréon et ses compagnons, martyrs. Fête : le 10 octobre.

(309) Maître Jean dit de la Longue Rue est cité le 24 décembre 1318, le 8 février 1322 et le 24 août 1333. Il était décédé le $1^{\text {er }}$ octobre 1360 . Cf. Renardy, Les maitres universitaires, p. 357. - LAHAYE, Saint-Materne, p. 109. - C.S.L., t. 3, pp. 187, 234, 423; t. 4, p. 328. 
Triumphus Sancti Lamberti (310). Totum duplex.

XII G II idus

(14 octobre)

Calixti (311) pape et martyris $\left({ }^{a}\right)$. Collecta $\left({ }^{b}\right)$.

Commemoratio domini Bastiani de Gluns (312), nostri concanonici et presbyteri, pro quo habemus IIII modios spelte.

I A idus

(15 octobre)

Sanctorum Maurorum (313) martyrum. Collecta.

Galli (314) abbatis et confessoris. Commemoratio.

IX C XVI kalendas

(17 octobre)

Commemoratio Gerardi, condam vicarii de Villari Episcopi, pro quo habemus I modium spelte hereditarie super domo et curte una sita in dicta villa prope le Flox (315).

$\left({ }^{a}\right)$ cum sociis suis, suscrit. $-\left({ }^{b}\right)$ missa, suscrit.

(310) Triomphe de saint Lambert à Steppes (1213). Fête : le 13 octobre.

(311) Saint Calixte ${ }^{\text {er }}$, pape, martyr. Fête : le 14 octobre.

(312) Maitre Bastien de Glons, chanoine de Saint-Materne, est cité du 3 février 1314 au 3 décembre 1336. Son testament est daté du 3 novembre 1334. Cf. ReNdRDY, Les mấtres universitaires, p. 189. - C.S.L., t. 3, pp. 187 n. 3, 188, 210, 464-465 - PONCELET, F.A.M., pp. 126, 433. Il est commémoré le 14 octobre dans l'O.S.L., éd. MarCHandisse, p. 140 et n. 881 .

(313) Les Maures, martyrs CCCLX (CCC). Fête : le 15 octobre.

(314) Saint Gall, conf. Fête : le 16 octobre.

(315) Floxhe, lieu-dit à Vottem (pr. Liège, arr. Liège, comm. Herstal). 
Commemoratio domini Gerardi Melter (316), concanonici nostri, pro quo habemus III modios VI sextarios spelte $\left({ }^{a}\right)$.

D XV kalendas

(18 octobre)

Luce (317) evangeliste. Duplex. Mononis (318) martyris. Commemoratio.

Commemoratio $\left({ }^{b}\right)$ domini Johannis Digma (319), confratris nostri, qui nobis legavit omnia sua antiqua debita unatum suo anno gratie necnon $V$ modios spelte hereditarie et $X$ florenos colonienses solutos ad redeficationem nostrarum domorum in Gerardria $\left(^{(}\right)(320)$.

$\left[41 r^{\circ}\right]$ XVII E XIIII kalendas

(19 octobre)

VI $\quad$ F XIII kalendas

(20 octobre)

Caprasii (321) martyris. Commemoratio.

$\left(^{a}\right) E$ : écriture de type littera cursiva $\left(X V^{e} s.\right) .-\left({ }^{b}\right)$ Le mot est interpolé. La fin de la commémoration sanctorale $(\mathrm{com})$ a été employée comme début de la notice-obituaire. (c) D: écriture de type littera cursiva formata (fin $X V^{e}$ s.).

(316) Gérard Melter reçut sa prébende sous-diaconale à Saint-Materne le 3 novembre 1417 et est cité comme chanoine de 1417 à sa mort, le 17 octobre 1427. Cf. LAHAYE, Saint-Jean, t. 1, p. 362 ; t. 2, p. 104. - LAHAYE, Saint-Materne, p. 147.

(317) Saint Luc, évangéliste, conf. Fête : le 18 octobre.

(318) Saint Monon, ermite, martyr. Fête : le 18 octobre, à Liège.

(319) Jean de Dyck, d'Eyck ou Digma est cité le 27 avril 1438 comme bénéficier de l'église de Tongres; le 20 novembre 1439 comme chapelain représentant l'Aumône de la Cathédrale; le 16 mai 1444 également comme chapelain de Saint-Lambert. Il devint chanoine sous-diacre de Saint-Materne le 20 février 1454 et est cité comme tel ou comme receveur de l'Aumône de la Cathédrale du 7 décembre 1458 au 21 juin 1480 . Il présenta son testament au chapitre le 27 novembre 1480 et mourut en 1482. Cf. LAHAYE, Saint-Materne, p. 143. - C.S.L., t. 5, pp. 119, 128, 160, 161, 164, 166, 168, 169, 196, 209.

- Paquay, Cartulaire N.-D. Tongres, t. 2, p. 151.

(320) Gérardrie. Cf. note 126.

(321) Saint Caprais, martyr. Fête : le 20 octobre. 
G XII kalendas

(21 octobre)

Undecim milium virginum (322). IX lectiones.

Commemoratio domini Gerardi de Cortenbach (323) pro quo habuimus $L$ florenos renenses semel solutos, ad opus redificationis nostrarum domorum in Gerardria (324) per manus domini Cristiani de Weert (325), sui capellani et confratris nostri.

Eodem die, commemoratio domini Cristiani Cortenbach, confratris nostri, qui emit $V$ modios IIII sextarios spelte in granario nostro, pro quibus solvit $L X$ florenos renenses colonienses conversos per confratres nostros ad perfectionem et constructionem horrei nostri in Vilari (326) pro decima colligenda et conservanda. Qui quidem confrater noster adiecit conditionem illam ut hii V modii et IIII sextarii spelte distribuantur inter presentes in promptu hoc die anniversarii generosi domini Gerardi de Cortembach, domini sui, ipso veniente pro nunc et post ejus decessum ab humanis perpetuis temporibus pro anima ejus et animabus parentum benefactorum et omnium Christi fide$\operatorname{lium}\left({ }^{a}\right)$.

(a) $I d$.

(322) Les onze mille Vierges. Fête : le 21 octobre.

(323) Gérard de Cortembach était chanoine d'Aix-la-Chapelle et de la collégiale Saint-Denis de Liège en 1427. Il résida comme chanoine de Saint-Lambert et comme abbé de Visé de 1431 à 1456. Il mourut le 21 octobre 1471. Cf. De TheuX, S.L., t. 2, p. 219. - Paquay, Cartulaire N.-D. Tongres, t. 2, p. 187. - C.S.L., t. 5, p. 192. - FaIRON, Regestes, t. 4, pp. 98, 106, 158. Des dispositions testamentaires sont évoquées dans Bormans, Conclusions capitulaires, p. 29. Pour son épitaphe à la Cathédrale, cf. NaveauPoullet, t. 1, p. 28.

(324) Gérardrie. Cf. note 126.

(325) Christian de Weerdt, chanoine (diacre) de Saint-Materne. En 1450-1451, il partage les revenus de la deuxième prébende diaconale de Saint-Materne avec les exécuteurs testamentaires d'Arnold de Bouchout, revenus qu'il perçoit pour huit mois. Il siégea plus de 50 ans. Il est cité comme chanoine de Saint-Materne le 21 juin 1480 et le 12 juillet 1485. Son testament fut approuvé le 24 février 1502. Cf. LaHAye, Saint-Materne, p. 129.

- C.S.L., t. 5, pp. 192, 209, 221.

(326) Villers-l'Évêque. Cf. note 25. 
$\left[41 v^{\circ}\right]$ XIIII A XI kalendas

(22 octobre)

III B X kalendas

( 23 octobre)

Severini (327) episcopi et confessoris. IX lectiones.

C IX kalendas

(24 octobre)

Ode (328) vidue. IX lectiones.

$\left[42 r^{\circ}\right]$ XI D VIII kalendas

(25 octobre)

Crispini et Crispiniani (329) martyrum. Collecta.

Commemoratio Goblionis (330), concanonici nostri, qui legavit nobis $\mathrm{X}$ modios spelte in granario nostro.

XIX E VII kalendas

(26 octobre)

Amandi (331) episcopi et confratris. Commemoratio.

Commemoratio domini Nicholai Grucheir (332), concanonici nostri et presbyteri, pro quo habemus XV solidos

(327) Saint Séverin, évêque, conf. Fête : le 23 octobre.

(328) Sainte Ode, veuve. Fête : le 23 ou le 24 octobre, à Liège.

(329) Saints Crépin et Crépinien, frères martyrs. Fête : le 25 octobre.

(330) Goblion, chanoine de Saint-Materne, est cité dans les actes de novembre 1237 au 13 avril 1272. Il intervint notamment comme arbitre dans un conflit opposant le chapitre de la collégiale Saint-Jean l'Évangéliste à son prévôt. Cf. C.S.L., t. 1, p. 392; t. 2, p. 72. - LAHAYE, Saint-Jean, t. 1, pp. 80, 98. Il est commémoré le 24 octobre dans le Polyptyque de Saint-Lambert, pp. 170-171 et le 25 octobre dans l'O.S.L., éd. MarCHaNDISSE, p. 146 et n. 929.

(331) Saint Amand, évêque, martyr, conf. Fête : le 26 octobre.

(332) Nicolas Grucheir, chanoine de Saint-Materne, est cité le 26 août 1333 mais n'est plus mentionné le $1^{\text {er }}$ octobre 1360. Cf. LaHAYE, Saint-Materne, p. 109. - C.S.L., t. 3 , p. 423 ; t. 4 , pp. 328 , 444. Il est commémoré le 27 octabre dans l'O.S.L., éd. MARCHANDISSE, p. 148 et n. 943. 
bone monete supra domum unam sitam iuxta portam Pontis Avroti (333).

F VI kalendas

(27 octobre)

Rumoldi (334) episcopi et martyris. Collecta. Vigilia.

Commemoratio domini Jacobi Bocherul (335). XXXVIII solidi bone $\left({ }^{a}\right)$.

$\left[42 v^{\circ}\right]$ VIII G V kalendas

(28 octobre)

Symonis et Judae (336) apostolorum. Totum duplex.

A IIII kalendas

(29 octobre)

Narcisci (337) episcopi et martyris. Commemoratio.

Commemoratio Gerardi de Bomalia (338), canonici Leodiensis, pro quo habemus in anniversario ipsius, III modios et dimidium spelte super terram quam emimus apud Limont (339).

( $\left.{ }^{a}\right) D$ : écriture de type littera cursiva formata $\left(X I V^{e}-X V^{e}\right.$ s.).

(333) Pont d'Avroy, rue de Liège. Cf. Gobert, Les rues de Liège, t. 5, pp. 36-45.

(334) Rumoldus (Rombaldus), évêque, martyr. Fête : le 27 octobre, à Liège.

(335) Jacques Bocheroul est cité le $1^{\text {er }}$ octobre 1360, le 2 mai 1363, le 15 décembre 1366, comme chanoine de Saint-Materne. Il était décédé le 22 décembre 1372. Cf. C.S.L., t. 4, pp. 328, 376, 444, 507. - TiHon, Lettres Grégoire XI, t. 2, n 1941, p. 228.

(336) Saints Simon et Jude, apôtres. Fête : le 28 octobre.

(337) Saint Narcisse ou Narciscus, évêque (conf.), martyr. Fête : le 29 octobre.

(338) Gérard de Bomal, chanoine de Saint-Lambert, est cité de 1215 à 1254. Son testament est daté de mai 1256. Il mourut en 1259. Cf. ChOt-STASSART, p. 62. - É. PonCeLET, Le monastère de Bernardfagne dit de Saint-Roch, B.S.A.H.D.L., t. 13, 1902, p. 205. Il est commémoré le 30 octobre dans l'O.S.L., éd. MakCHaNDisse, p. 150 et n. 953 et dans le Polyptyque de Saint-Lambert, p. 171. Pour son épitaphe à l'abbaye de Robermont, cf. Naveau-Poullet, t. 2, p. 16.

(339) Limont (pr. Liège, arr. Waremme, comm. Donceel). 
XVI B III kalendas

(30 octobre)

V C II kalendas

(31 octobre)

Quintini (340) et Foillani (341) martyrum. Collecta. Vigilia.

Commemoratio Willermi de Fiez, concanonici nostri, et Reneri dicti Wasar, monachi fratris sui, et Agnetis, matris eorumdem, pro quibus habemus II modios siliginis in territorio de Horpale (342).

NOX HABET HORAS XIIII, DIES X

$\left[43 r^{\circ}\right]$ QUINTA NOVEMBRIS ACUS VIX TERTIA MANSIT IN URNA

NOVEMBER HABET DIES XXX, LUNA XXVII

D kalendas

(1 novembre)

Festivitas Omnium Sanctorum. Totum duplex.

Commemoratio Arnuldi de Hollant (343), canonici Leodiensis, pro quo habemus ultra Mosam (344), XXVII solidos VII denarios obolum bone monete.

Commemoratio domini Hermanni de Zanctis (345), pres-

(340) Saint Quentin, martyr. Fête : le 31 octobre.

(341) Saint Feuillien de Fosses, martyr. Fête : le 31 octobre.

(342) Horpmaal, commune de Heers (pr. Limbourg, arr. Tongres, comm. Heks).

(343) Arnoul de Hollande, chanoine de Saint-Lambert, apparaît sous son nom complet de 1215 au 11 avril 1236, date à laquelle il était décédé. C'est peut-être également lui qui est cité par son seul prénom en 1203 et en 1209. Cf. Chot-STassart, p. 82. C.S.L., t. 1, pp. 357-358 - PAQUaY, Cartulaire N.-D. Tongres, t. 1, p. 69. - H. DE L'EsCAILlE, Notice sur les seigneuries de Vyle et Tharoul, B.I.A.L., t. 16, 1881, p. 481.

(344) Outremeuse. Cf. note 107.

(345) $\mathrm{Cf}$. note 4 . 
byteri canonici Leodiensis, pro quo habemus I modium spelte in granario nostro $\left({ }^{a}\right)$.

XIII E IIII nonis

(2 novembre)

Commemoratio animarum (346). Eustacii (347), cum sociis suis, martyrum. III lectiones.

Commemoratio omnium benefactorum nostrorum et defunctorum pro quibus habemus in granario nostro III modios spelte distribuendos in promptu presentibus in vigiliis, de quibus legavit Johannes Leonardi (348) II modios et pro domino Henrico Laecman(349) I modium spelte $\left(^{b}\right)$.

II $\quad$ F III nonis

(3 novembre)

Huberti (350) episcopi et confessoris. Duplex.

Commemoratio Yde de Puteo, matris domini Henrici Oneal (351) concanonici nostri, pro qua habemus VI sextarios spelte apud Villari Episcopi (352) de quibus habemus litteras in cophinio nostro $\left({ }^{c}\right)$.

${ }^{(a)} I d .-\left({ }^{b}\right) E$ : écriture de type littera cursiva $\left(X V^{e}\right.$ s.). $-\left({ }^{c}\right) I d$.

(346) Fête des âmes: le 2 novembre.

(347) Saint Eustache et ses compagnons, martyrs. Fête: le 2 novembre.

(348) Jean Leonard(i) ou Lynar succéda à Guillaume de Heers comme chanoine sous-diacre de Saint-Materne. Il est cité à ce titre du $1^{\text {er }}$ juillet 1446 à novembre 1470 . Il produisit son testament au chapitre de Saint-Materne, le 7 août 1483. Cf. LaHAYE, Saint-Materne, p. 139. - C.S.L., t. 6, pp. 180, 185, 188, 195.

(349) Cf. note 48.

(350) Saint Hubert, évêque, conf. Fête : le 3 novembre.

(351) Cf. note 24.

(352) Villers-l'Évêque. Cf. note 25. 
Perpetui (353) et Amandi (354) episcoporum et confessorum. Collecta.

Commemoratio domini Goberti de Sancto Servatio, presbyteri, pro quo habemus II modios spelte.

$\mathrm{X} \quad \mathrm{A}$ nonis

(5 novembre)

B VIII idus

(6 novembre)

Leonardis (355) abbatis et confessoris. IX lectiones.

$\left[44 r^{\circ}\right]$ XVIII C VII idus

(7 novembre)

Willebrordi (356) episcopi et confessoris. Collecta.

VII D VI idus

(8 novembre)

Quatuor coronatorum (357) martyrum. Collecta et missa.

E V idus

(9 novembre)

Theodori $\left({ }^{a}\right)(358)$. Collecta et missa.

$\left({ }^{a}\right)$ Les mots episcopi et sont biffés.

(353) Saint Perpète, évêque, conf. Fête : le 4 novembre.

(354) Saint Amand, évêque, conf. Fête : le 4 novembre. À son propos, cf. J. Dufour, Les évêques d'Albi, de Cahors et de Rodez, des origines à la fin du XIT siècle, Paris, 1989 , pp. 73-74.

(355) Saint Léonard, abbé, conf. Fête : le 6 novembre.

(356) Saint Willibrord, évêque, conf. (martyr). Fête : le 7 novembre.

(357) Les quatre saints couronnés (Sévère, Séverin, Victorin, Carpofore), frères martyrs. Fête : le 8 novembre.

(358) Saint Théodore, martyr. Fête : le 9 novembre. 
Martini (359) pape et martyris. Commemoratio.

Commemoratio Egidii de Prella (360), subdyaconi nostri concanonici, pro quo habemus $\mathrm{XX}$ solidos bone. Item $\mathrm{XX}$ sextarios spelte.

IIII G III idus

(11 novembre)

Martini (361) episcopi et confessoris. Duplex. Mennae (362) martyris. Collecta. Bertuini (363) episcopi et confessoris. Commemoratio.

A II idus

(12 novembre)

Cuniberti (364) episcopi et confessoris. Commemoratio.

B idus

(13 novembre)

Brictii (365) episcopi et confessoris. IX lectiones.

Anno Domini XIIII $L X X X^{\circ}$, mensis novembris, die, obiit honorabilis vir Johannes de Wellynes (366), quondam

$\left.{ }^{(}\right)$En bas du $f^{\prime} 44 v^{\circ}$, taches et griffonnages.

(359) Saint Martin $\mathrm{I}^{\text {er }}$, pape, martyr. Fête : le 10 novembre.

(360) Gilles de Presles, chanoine de Saint-Materne, cité du 26 janvier 1264 (n. st.) à février 1308 (n. st.). Cf. G. KunTh, Chartes de l'abbaye de Saint-Hubert en Ardenne, t. 1, Bruxelles, 1903, p. 375. - C.S.L., t. 2, pp. 382, 485 ; t. 3, p. 85. - ScHOONBROODT, Val-Saint-Lambert, t. 1, p. 114. Il est cité dans le Polyptyque de Saint-Lambert, pp. 79, 80, et est commémoré le 7 novembre dans l'O.S.L., éd. MarChandisSe, p. 156 et n. 992.

(361) Saint Martin, évêque, conf. Fête : le 11 novembre.

(362) Saint Menne, martyr. Fête : le 11 novembre.

(363) Saint Bertuin, conf. Fête: le 11 novembre, à Liège uniquement.

(364) Saint Cunibert, évêque, conf. Fête : le 12 novembre.

(365) Saint Brice (Bris, Bres), évêque, conf. Fête : le 13 novembre.

(366) Jean de Wellynes, clerc, citain de Liège, homme de la cour allodiale et receveur de la Grande Compterie de la Cathédrale, est cité du 11 juin 1441 au 20 décembre 1476. Il mourut, semble-t-il, le 13 novembre 1480 . Cf. C.S.L., t. 5, pp. 122, 129, 141, 142, 143, $150,185,186,190,194,195,197,202,234,250$; t. 6, p. 197. - CUVELIER, Inventaire Val-Benoît, pp. 299, 307, 314. - Adrien d'Oudenbosch, Chronique, éd. C. de Borman, Liège, 1902, p. 218. Selon LAHAYE, Saint-Materne, p. 117, Jean de Wellynes aurait été 
computator Sancti Lamberti, qui legavit fabrice ecclesie Leodiensis $X X$ marchas census bone hereditarie quas habuit hereditarie supra domum dictam dez Vingnerons in Foro Leodiensis situatam. Quos tenentur solvere hereditarie Petrus de Equo (367) quondam tam Martinus de Viridi Equo (368) commorans in parochia Sancti Severini (369) Leodiensis. Ex quibus XX marchis census legavit idem testator canonicis Sancti Materni III marchas, canonicis Parve Mense, similiter III marchas, beneficiatis ecclesie Leodiensis similiter III marchas et fraternitati Sancti Luce (370) prope scolas ecclesie III marchas census hereditarie faciendus. Similiter XII marchas census quos fabrica dicte ecclesie tenetur solvere membrorum prescriptis communibus annis hereditarie seu computatoris dicte fabrice ${ }^{a}$ ).

${ }^{(a)}$ D : écriture de type littera cursiva formata (début $X V I^{e}$ s.).

nommé chanoine de Saint-Materne en juillet 1398 et aurait été admis à prendre possession de sa charge le 9 septembre 1400. Faire du chanoine et du receveur une seule et même personne serait lui accorder une carrière d'une étonnante longévité (de 1398 à $1480)$.

(367) Le 19 juin 1450, Jean de Wellynes, clerc et citain de Liège (cf. note précédente), fait savoir qu'il a donné à Pierre de Cheval, clerc, la maison des Trois-Pierres, au coin de Féronstrée et de la rue du Pont. Cf. C.S.L., t. 5, p. 143. Pierre de Cheval, clerc, citain de Liège, maire de la cour des tenants du Val-Benoît, compteur et receveur de cette abbaye, est cité du 19 juin 1450 au $1^{\text {er }}$ février 1507 . Il était décédé le 3 janvier 1509 . Cf. C.S.L., t. 5, p. 143. - CuVelier, Val Benoît, pp. 492, 494. - Cuvelier, Inventaire Val-Benoit, pp. 327, 328, 331, 332, 333, 334, 337, 344, 349, 350, 356, 357, 358, 359, 360, $364,371,374,389,436,441$.

(368) Martin du Vert Cheval, brasseur, mambour du Val-Benoît, est cité dans un record donné par la cour des tenants du Val-Benoît et daté du 8 juillet 1503 . Cf. CuveLIER, Val-Benoît, p. 491.

(369) Saint-Severin, paroisse et église de Liège. Cf. Gobert, Les rues de Liège, t. 5, pp. 324-333. - Lahaye, Paroisses, p. 14 et passim.

(370) La confrérie de Saint-Luc fut fondée au XIII ${ }^{\mathrm{e}}$ siècle par Jean de Moregny, doyen de Saint-Lambert (cf. note 119). Elle avait pour but d'apporter à ses membres trente, dont sept chanoines tréfonciers, quatre chanoines de Saint-Materne, quatre chanoines de la Petite-Table et quinze bénéficiers - une aide efficace, en cas de maladie, et une assistance spirituelle. À son propos, cf. É. SchoolmeEsTERS, Jean de Moregny et la confrérie de Saint-Luc, Leodium, t. 9, 1910, pp. 37-42. - A. DuBors, Le chapitre cathédral de Saint-Lambert à Liège au XVIr siècle, Liège, 1949, pp. 170-171. 
Commemoratio domini Theoderici Rotarii (371), concanonici nostri, pro quo habemus apud Voruz (372) prope Lieerses $\left({ }^{a}\right)$ (373) I modium spelte hereditarie de quo habemus litteram in cophinio nostro $\left({ }^{b}\right)$ de $X X$ sextariis emptis anno plurimo, mensis augusti, erga Collino de Worouz $\left(^{c}\right)(374)$.

D XVII kalendas (15 novembre)

Eugenii (375) episcopi et martyris. Commemoratio.

Commemoratio Marchi, concanonici nostri, pro quo habemus XI solidos bone $\left({ }^{d}\right)$.

Othmari $\left(^{e}\right)(376)$ confessoris. Commemoratio.

F XV kalendas (17 novembre)

Aniani (377) episcopi et confessoris. Commemoratio.

$\left({ }^{a}\right)$ Le toponyme pourrait également être Bieerses. $-\left({ }^{b}\right)$ Un mot illisible. $-\left({ }^{c}\right) E$ : écriture de type littera cursiva $\left(X V^{e} s.\right)$. - (d) Sous la commémoration apparaît le nombre $18287 .-\left({ }^{(}\right)$Les mots episcopi et sont biffés.

(371) Thierry Rotarii, chanoine (prêtre) de Saint-Materne, fut nommé en 1410. Il est cité avec ce titre le 10 janvier 1426 et le 14 octobre 1427. Le 10 juillet 1440, il est admis comme chanoine de la collégiale Sainte-Croix de Liège mais cette admission est annulée le 12 décembre 1440. Il meurt le 15 novembre 1441. Cf. LAHAYE, Saint-Materne, p. 121. - C.S.L., t. 6, pp. 170, 171. - Poncelet, Sainte-Croix, t. 1, p. ClXV.

(372) Si le toponyme qui suit est Lieerses, il s'agit de Voroux-lez-Liers (pr. Liège, arr. Liège, comm. Juprelle); si, par contre, il s'agit de Bieerses, Voruz correspond à VorouxGoreux (pr. Liège, arr. Waremme, comm. Fexhe-le-Haut-Clocher).

(373) Liers (pr. Liège, arr. Liège, comm. Herstal) ou Bierset (pr. Liège, arr. Liège, comm. Grâce-Hollogne).

(374) Cf. note 372.

(375) Saint Eugène, évêque, martyr. Fête : le 15 novembre.

(376) Saint Otmar, abbé, conf. Fête : le 16 novembre.

(377) Saint Agnan (Aignan), évêque, conf. Fête : le 17 novembre. 
Commemoratio domini Johannis de Machlinia (378), canonici Leodiensis et subdyaconi, pro quo habemus I modium spelte apud Vorous (379) supra.

XVII G XIIII kalendas

(18 novembre)

Octava Sancti Martini ( $\left.{ }^{(}\right)$. III lectiones. Severi (380) confessoris. Commemoratio.

[46r ${ }^{\circ}$ VI A XIII kalendas

(19 novembre)

Elyzabeth (381) vidue. III lectiones. Gelasii (382) pape et confessoris. Commemoratio.

B XII kalendas

(20 novembre)

XIIII C XI kalendas

(21 novembre)

Columbani (383) abbatis et confessoris. Commemoratio.

$\left[46 v^{\circ}\right]$ III

D X kalendas

(22 novembre)

Cecilie (384) virginis et martyris. IX lectiones.

E IX kalendas

(23 novembre)

Clementis $\left(^{b}\right)(385)$ martyris. IX lectiones. Felicitatis (386) martyris. Collecta.

$\left({ }^{a}\right)$ confessoris, suscrit. $-\left({ }^{b}\right)$ pape et, suscrit.

(378) Cf. note 68

(379) Cf. note 20.

(380) Saint Sévère, évêque, conf. Fête : le 18 novembre.

(381) Sainte Élisabeth de Hongrie, $\uparrow 1231$, canonisée en 1235 . Fête : le 19 novembre.

(382) Saint Gélase I ${ }^{\mathrm{er}}$, pape, conf. Fête : le 19 novembre.

(383) Saint Colomban, abbé, conf. Fête : le 21 novembre.

(384) Sainte Cécile, vierge et martyre. Fête : le 22 novembre.

(385) Saint Clément $I^{\text {er }}$, pape, martyr. Fête : le 23 novembre.

(386) Sainte Félicité, martyre avec ses sept fils. Fête: le 23 novembre. 
XI F VIII kalendas

(24 novembre)

Trudonis (387) confessoris. IX lectiones $\left({ }^{a}\right)$.

Commemoratio domini Galteri (389), decani Leodiensis et abbatis Sancte Marie, qui instituit $X$ canonicos in ecclesia Beate Marie (390), pro quo habemus V modios et dimidium spelte in granario nostro et fit dictum anniversarium in hospitali novo supra Mosam (391) in quo corpus dicti decani jacet et requiescit.

$\left[47 r^{\circ}\right]$ XIX G VII kalendas

( 25 novembre)

Katherine (392) virginis $\left({ }^{b}\right)$. Duplex. Petri (393) episcopi et martyris. Commemoratio.

A VI kalendas

(26 novembre)

Lini (394) pape et martyris. Collecta.

$\left({ }^{a}\right)$ Crisogoni (388) martyris. Collecta, suscrit. $\quad\left({ }^{b}\right)$ et martyris, suscrit.

(387) Saint Trudon, conf., presbyter. Fête : le 23 ou le 24 novembre, à Liège.

(388) Saint Chrysogone, martyr. Fête : le 24 novembre.

(389) Maître Gauthier de Chauvency est cité dès 1171 (comme diacre), en 1176, en 1178, en 1184 (comme prévôt de Fosses), en 1185, 1186 (comme prévôt de Fosses), en 1189 (comme écolâtre de Saint-Lambert), en 1192 (comme archidiacre), en 1194 (comme écolâtre et archidiacre), en 1196 (comme archidiacre), en 1197 et de 1198 à 1207 comme doyen de Saint-Lambert et archidiacre. Il est abbé de Notre-Dame-aux-Fonts de 1200 à 1207. Il est à l'origine de la création du chapitre de Saint-Materne et de la fondation de l'hôpital de Saint-Mathieu-à-la-Chaîne. Il mourut le 22 novembre 1207. Cf. RenaroY, Les maitres universitaires, pp. 122-124.

(390) Notre-Dame-aux-Fonts, chapelle de la Cathédrale de Liège, affectée au ministère paroissial. Cf. LaHaye, Paroisses, pp. 1-13.

(391) Hôpital de Saint-Mathieu-à-la-Chaîne, hôpital de Liège, situé dans les cloîtres de la Cathédrale. Cf. P. DE SPIEGELer, Les hôpitaux et l'assistance à Liège ( $X-X V^{e}$ siècles). Aspects institutionnels et sociaux, Paris, 1987, pp. 65-69. Cf. également

U. BERLIÈRE et autres, Monasticon belge, t. 2, Maredsous, 1928-1955, pp. 384-387.

(392) Sainte Catherine, vierge et martyre. Fête : le 25 novembre.

(393) Saint Pierre, évêque, martyr. Fête : le 25 novembre.

(394) Saint Lin, pape, martyr. Fête : le 26 novembre. 
VIII B V kalendas

(27 novembre)

$\left[47 v^{\circ}\right]$

C IIII kalendas

(28 novembre)

XVI D III kalendas

(29 novembre)

Saturnini (395), cum sociis suis, martyrum. Collecta. Vigilia.

V E II kalendas

(30 novembre)

Andree (396) apostoli. Duplex.

NOX HABET HORAS XVI, DIES VIII

\section{$\left[48 r^{\circ}\right]$ DAT DUODENA COHORS SEPTEM, INDE DECEMQUE DECEMBER (397)}

\section{DECEMBER HABET DIES XXXI, LUNA XXIX}

F kalendas

(1 décembre)

Eligii (398) episcopi et confessoris. Collecta.

Commemoratio domini Hermanni de Zanctis (399), presbyteri canonici Leodiensis, pro quo habemus I modium spelte in granario nostro $\left({ }^{\circ}\right)$.

$\left.{ }^{a}{ }^{a}\right)$ D : écriture de type littera cursiva formata $\left(X I V^{e}-X V^{e} s.\right)$.

(395) Saint Saturnin, évêque, et ses compagnons [Saturninus, Chrysanthus, (Maurus) et Daria] martyrs. Fête : le 29 novembre.

(396) Saint André, apôtre. Fête : le 30 novembre.

(397) La forme habituelle est Dat duodena cohors septem, inde decemque decembris. Cf. Grotefend, Zeitrechnung, t. 1, p. 36 et ici-même, pp. 25-27.

(398) Saint Éloi, évêque, conf. Fête : le $1^{\text {er }}$ décembre.

(399) Cf. note 4. 
II.XIII G IIII nonis

(2 décembre)

Commemoratio Beatricis pro qua habemus III modios spelte in granario nostro.

A III nonis

(3 décembre)

Commemoratio Rodulphi, concanonici nostri, pro quo habemus I modium siliginis in granario nostro.

B II nonis

(4 décembre)

Barbare (400) virginis et martyris. IX lectiones. Duplex.

C nonis

(5 décembre)

Commemoratio domini Anthonii Muselere (401), concanonici nostri, de quo habemus I modium spelte hereditarie apud Woruz (402) ut patet in littera $\left(^{a}\right)$ quam habemus in cophinio nostro $\left({ }^{b}\right)$ de $X X$ sextariis spelte $\left({ }^{c}\right)$.

XVIII D VIII idus

(6 décembre)

Nicolai (403) episcopi et confessoris. Duplex.

E VII idus

(7 décembre)

Octava Sancte Andree (404) apostolis. III lectiones.

$\left({ }^{a}\right)$ de, biffé. $-\left({ }^{b}\right)$ Un mot illisible. $-\left({ }^{c}\right)$ E : écriture de type littera cursiva $\left(X V^{e} s.\right)$.

(400) Sainte Barbe, vierge, martyre. Fête : le 4 décembre.

(401) Antoine Museler remplaça Thierry Bucke $(\dagger 1411)$ comme chanoine prêtre de Saint-Materne. Il resta en fonction jusqu'au 5 décembre 1442, jour de sa mort. Cf. LAHAYE, Saint-Materne, p. 114.

(402) Voroux-lez-Liers ou Voroux-Goreux. Cf, note 20.

(403) Saint Nicolas, évêque, conf. Fête : le 6 décembre.

(404) Cf. note 396. L'octave est célébrée le 7 décembre. 
Commemoratio Colardi de Meffia (405), subdyaconi concanonici nostri, pro quo habemus $\mathrm{V}$ solidos bone.

F VI idus

(8 décembre)

Conceptio Beate Marie virginis (406). Totum duplex.

XV G V idus

(9 décembre)

$\left[49 v^{\circ}\right]$ IIII A IIII idus

(10 décembre)

B III idus

(11 décembre)

Damasi (407) pape et confessoris. Collecta.

XII C II idus

(12 décembre)

Commemoratio domini Jacobi de Sarto (408), canonici Sancti Dyonisii et presbyteri ac dudum canonici Sancti Materni, pro quo habemus III modios spelte recipiendos a provisore fabrice ecclesie Leodiensis et debent fieri due candele de dimidia libra cere que ardere debent in capella Sancti Materni ab initio vigiliarum usque ad finem misse diei sequentis continue.

(405) Collard de Meeffe détenait un canonicat et une prébende à Saint-Materne le 28 juin 1352 . Il est cité le $1^{\text {er }}$ octobre 1360 mais n'est plus mentionné le 30 avril 1374. Cf. LAHAYE, Saint-Materne, p. 109. - C.S.L., t. 4, pp. 162, 328, 508.

(406) L'Immaculée Conception : le 8 décembre.

(407) Saint Damase I ${ }^{\text {er }}$, pape, conf. Fête : le 11 décembre.

(408) Jacques de Sart, chanoine de Saint-Denis, est cité le 19 et le 20 octobre 1326. Cf. Cuvelier, Val-Benott, pp. 377, 378, 381, 383. 
XIIII D XIII kalendas

(20 décembre)

Vigilia.

III E XII kalendas

(21 décembre)

Thome (409) apostoli. Duplex.

Commemoratio Egidii dicti le Frongut (410), dyaconi concanonici nostri, pro quo habemus III sextarios spelte.

XIX A IX kalendas

(24 décembre)

Vigilia.

Nativitas Domini nostri Jhesu Christi. Totum duplex.

VIII C VII kalendas

(26 décembre)

Stephani (411) prothomartyris. Totum duplex.

(409) Saint Thomas, apôtre. Fête : le 21 décembre.

(410) Gilles dit le Frongnut est cité comme clerc de Liège dans la Paix d'Angleur, le 14 février 1313 , et, sans titre, le 7 août 1313. Il est également mentionné comme clerc de Liège le 16 août 1318 et le 4 novembre 1328 ; comme clerc juré de la Cité de Liège, le 6 octobre 1321. Il était chanoine de Saint-Materne le 6 mai 1351 et était probablement décédé le 9 décembre 1353. Cf. C.S.L., t. 4, pp. 140, 181 ; t. 6, pp. 51, 311. - FAIron, Regestes, t. 1, pp. 524, 525-527. - E. Poswick, Documents inédits sur la haute avouerie de Hesbaye, B.I.A.L., t. 11, 1872, p. 193. - St. Bormans, Recueil des ordonnances de la principauté de Liège, Première série (974-1506), Bruxelles, 1878, p. 146 n. 2.

(411) Cf. note 5. 
D VI kalendas

Johannis evangeliste (412). Totum duplex.

Commemoratio domini Alberti (413), canonici Parve Mense et sacerdotis, pro quo habemus I modium spelte jacentem apud Nawerulle (414) super curtem unam et domum quam tenet relicta Hanetay et eius filia Elizabeth. Descendit autem illa curtis et domus de curia de Odor (415).

Commemoratio domini Henrici de Alken (416), presbyteri concanonici nostri, pro quo habemus apud Kemexhe (417), ut patet in registro nostro, $I X$ sextarios spelte $\left({ }^{a}\right)$.

$\left[51 v^{\circ}\right]$ XVI E V kalendas

(28 décembre)

Sanctorum Innocentium (418) martyrum. Totum duplex.

Commemoratio domini Cristiani de Gutphania $\left({ }^{b}\right)$, capellani ecclesie Leodiensis presbyteri, pro quo habemus $V$ solidos bone $\left({ }^{c}\right)$.

V $\quad$ F IIII kalendas

(29 décembre)

Thome (419) archiepiscopi et martyris. IX lectiones.

${ }^{(a)}$ id. $-\left({ }^{b}\right)$ Il s'agit peut-être de Zutphania (?). - ( $\left.{ }^{c}\right)$ E : écriture de type littera cursiva $\left(X I V^{e}-X V^{e}\right.$ s.).

(412) Cf. note 6.

(413) Albert, chanoine de la Petite-Table, est commémoré le 27 décembre dans l'O.S.L., éd. MarChaNdisSE, p. 174 et n. 1111.

(414) Naveroule, dépendance de Villers-l'Évêque (pr. Liège, arr. Liège, comm. Awans).

(415) Odeur (pr. Liège, arr. Waremme, comm. Crisnée).

(416) Cf. note 209.

(417) Kemexhe. Cf. note 210.

(418) Cf. note 7.

(419) Saint Thomas Becket, archevêque de Canterbury, martyr, $\dagger 1170$, canonisé en 1173. Fête : le 29 décembre. Sur ce culte, très précocement attesté à Liège, voir Chr. RENARDY, Notes concernant le culte de saint Thomas Becket dans le diocèse de Liège, R.B.P.H., t. 55, 1977, pp. 381-389. 
G III kalendas

(30 décembre)

IIII A II kalendas

(31 décembre)

Silvestri (420) pape et confessoris. III lectiones.

NOX HABET HORAS XVIII, DIES VI

(420) Saint Sylvestre $I^{\text {er }}$, pape, conf. Fête : le 31 décembre. 


\section{Index}

Aucune référence n'est donnée pour les noms Liège (en tant que localité) et Saint-Materne (désignant le chapitre et ses chanoines, en général), cités très souvent. Les fêtes n'ont pas été reprises, ni les noms de saints insérés dans ces désignations de fêtes (exemple: octave de la Saints-Pierre-et-Paul). Les anthroponymes et les toponymes difficilement traduisibles, sujets à discussion ou permettant plusieurs traductions ont, comme parfois dans l'édition, été maintenus en latin. Il en va de même de certains titres (presb.=presbyter; diac. = diaconus ; miles; famula). Lorsqu'il est question d'un chanoine ou d'un dignitaire de Saint-Barthélemy, de Saint-Denis, de Saint-Jean l'Évangéliste, de Saint-Lambert, de Saint-Materne, de Saint-Pierre, de Sainte-Croix ou de la Petite-Table, sans autre précision, il s'agit toujours de l'un des établissements religieux liégeois.

Abundus, Habundus (saint), 41, 84.

ACHILÉE, Achilleus (saint), 61.

Achilleus, voir ACHILÉE.

ADALBERT, Adalbertus (saint), 56.

Ada, voir ADAM.

ADAM, Ada, chanoine de SaintMaterne, 60.

Adaucte, Adauctus (saint), 85.

Adyncurt, toponyme non identifié, 84 .

AGAPET, Agapitus (saint), 81.

Agapitus, voir Agapet.

AGathe, Agatha (sainte), 39.

AgNan, Anianus (saint), 99.

AGNÈs (sainte), 36. - Mère de Willermus de Fiez, 94.

Aignan, voir AgNan.

AIx (Herbert d'), chanoine de Saint-

Lambert, 63. - (Jean d') junior, chanoine de Saint-Materne, 43. (Jean d') senior, chanoine de SaintMaterne, 43.

AIX-LA-Chapelle (R.F.A., RhénanieWestphalie). - Chanoine, voir Cortembach. - Chapitre, voir NOTRE-DAME.
Alain, Alvanus, voir LIÈGE.

Albamus, voir Aubain.

AlBert $I^{\text {er }}$ de Louvain, Albertus, évêque de Liège, 40. - Chanoine de la Petite-Table, 106.

Albertus, voir AlBERT.

AlBIN (saint), 24.

Aldegonde, Aldegundis (sainte), 37.

Aldegundis, voir ALDEGONDE.

Alexander, voir AlEXANDRE.

Alexandre I ${ }^{\mathrm{er}}$, Alexander (saint), pape, $24,59$.

AleXIS (saint), 79.

Alken (Henri d'), chanoine de SaintMaterne, 28, 72, 106.

Alleur (Liège, Liège, Ans), 46. Voir PuITs.

Alleur (Jean d'), parfois dit de Pu(i)che, chanoine de Saint-Materne, $46,47$.

Alvanus, voir AlaIN.

AmAND, Amandus (saint), fêté avec saint Vaast, 40. - (saint), évêque, 92. - (saint), évêque de Rodez, 96. 
Ambroise, Ambrosius (saint), 52.

Ambrosius, voir AMBrolse.

Amel, Amelius, voir Fize.

Amelius, voir AMEL.

Amor, voir AMOUR.

AMOUR, Amor (saint), 88.

ANaclet, voir Clet.

Anazar, voir NAZAIRE.

ANDRÉ, Andrea (saint), 102. - Frère de Jean de Enzivaux, 57. - Voir ÎLE, PINTE.

Andrea, voir ANDRÉ.

ANGLEUR (Paix d'), 105.

Anianus, voir AGNAN.

Anthonius, voir ANTOINE.

AntoIne, Anthonius, voir Fize, MoYLANT, MUSElER, NuCERIo.

Appollinaire, Appolinar, Appollinar (saint), 80. -- (saint), fêté avec saint Timothée, 83.

Appolonie (sainte), 24.

ApuléE, Apuleus (saint), 88.

ArLes (France, Bouches-du-Rhône).

- Concile, 12.

ArNold, voir Bouchout, Brouckem.

Arnoul, Arnuldus, Arnulphus (saint),

81. - Voir CoKiers, Hollande.

Arnould, Arnuldus, chanoine de la

Petite-Table, prédicateur, 69.

Arnuldus, voir ARnoul, ARNoUld.

Arnulphus, voir ARNOUL.

Aubain, Albanus (saint), 71.

Auctus, voir ADAUCTE.

Audactus, voir ADAUCTE.

Augustin, Augustinus (saint), philo-

sophe médiéval, $25,85$.

Avold, voir NABOR.

Avon, voir NABOR.

Balduinus, voir BAUDOUIN.

Banou, voir BANOW.

BANOW [Jean (de)], chanoine de Saint-

Materne, 62, 65. - Ses parents,

65.

Barbara, voir BARBE.
BARBE, Barbara (sainte), 103.

Barnaba, voir BARNABÉ.

BARNABé, Barnaba (saint), 67.

BARTHÉLEMY, Bartholomeus (saint),

84.

Bartholomeus, voir BARTHÉLEMY.

BASILE, Basilidis (saint), 24, 68.

Basilidis, voir BaSILE.

Basse-SAuvenière, rue de Liège, 83.

Bastianus, voir GLons.

Baudouin, Balduinus, voir Theux, TrimeIES.

BéATrice, Beatrix, 103. - Famula de maître Gauthier, 68.

Beatrix, voir BÉATRICE.

BÈde LE VÉnÉRable, philosophe médiéval, 25.

BENoîT DE NuRSIE, Benedictus (saint), 49, 77.

BERLIÈRE (dom Ursmer), historien belge, 2 .

Bermonbech, voir BERMONBÈCHE.

BERMONBÈche (Gilles, dit Fichon), chapelain puis chanoine de SaintDenis, chanoine de Saint-Pierre, de Saint-Lambert, abbé séculier de Maaseik, 76.

Bernard de Clairvaux, Bernardus (saint), 82.

Bernardus, voir BERNARD.

BERTUIN, Bertuinus (saint), 97.

Bieerses, voir BIERSET.

Bierses, voir BIERSET.

BIERSET, Bieerses, Bierses (Liège,

Liège, Grâce-Hollogne), 63, 99.

Blaise, Blasius (saint), 39.

Blasius, voir BlaIsE.

BLEHEN (Liège, Waremme, Hannut), 71.

BLEHEN (Herman de), chanoine de Saint-Materne, 77.

Bocheroul (Jacques), chanoine de

Saint-Materne, 21, 83.

Bocherul, voir BOCHEROUL.

Boechem, voir BROECHEM. 
Bomal (Gérard de), chanoine de

Saint-Lambert, 93.

Bomalia, voir BomaL.

Bonefacius, voir BonifaCE.

Boniface, Bonefacius (saint), 66. -

VIII, pape, 11.

Bons-Enfants, couvent de Liège, 84 .

- Prieur, 84.

Borgondi, voir BourguIGNoN.

Bouchout (Arnold de), chanoine de

Saint-Materne, 92.

Bouillon (Elie de), chanoine de

Saint-Lambert, écolâtre de Tirlemont, 22, 28, 40, 50, 55 .

Bourguignon (Jean le), 48. - (Jean

le), chanoine de Saint-Materne,

48. - (Josse le), chanoine de

Saint-Materne, 48. - (Pierre le), 76.

BouviGNes (Godefroid de), frère de

Jean de Bouvignes, 51. -... (Jean de), doyen de Sainte-Croix, 51.

Bovingue, voir Bouvignes.

BRABANT, duché. - Chancelier du duc, voir SART.

Bréda (Jean de), chanoine de SaintMaterne, 77.

Bres, voir BRICE.

Bresine Bastin, maison de Liège, 35.

BRICE, Brictius (saint), 97.

Brictius, voir BRICE.

BRIGIDE, Brigida (sainte), 23, 38.

Bris, voir BRICE.

Broechem (Brunon de) ou de Bruxhem, chanoine et compteur de Saint-Lambert, prévôt de SaintRombaut de Malines, 68.

Brono, voir BRUNON.

Brouckem (Arnold de), chanoine de

Saint-Materne, 16.

Bruges (Jean de), chanoine de Saint-

Materne, 80.

BRUNON, Brono, voir BROECHEM.

Bruxhem, voir BROECHEM.
BuCke (Thierry), chanoine de SaintMaterne, 43, 103.

Buke, voir BUCKE.

Buten (Guillaume de), chanoine de Saint-Materne, 46.

Caliga, voir Chausse.

Calixte I $^{\text {er }}$, Calixtus (saint), pape, 89.

Canistrata, voir ChInstréE.

CaPelle (Jacques), chanoine de SaintMaterne, 42.

Caprais, Caprasius (saint), 90.

Caprasius, voir CAPRAIS.

Casale (Ubertin de), franciscain spirituel italien, 20.

Catherine, Katherina (sainte), 101 .Sœur de Jean de Enzivaux, 57.

CécIle, Cecilia (sainte), 100.

Cecilia, voir Cécile.

Celles (Namur, Dinant, Houyet). Abbé, voir HADELIN (saint).

Снавот (Gérard), dit d'Ochain, doyen de Saint-Denis, chanoine de Saint-Paul de Nivelles, chanoine de Saint-Lambert, prévôt de SaintPaul de Liège, abbé séculier de Ciney, 37.

Chache, voir CHAusse.

Chapelle-des-Clercs, rue de Liège, 57.

Chauche, voir Chausse.

Chausse, Caliga (maison delle), maison de Liège, 57.

Chauvency (Gauthier de), doyen et écolâtre de Saint-Lambert, archidiacre, abbé de Notre-Dame-auxFonts, prévôt de Fosses, 9, 10, 11, 101.

Cheval (Pierre de), clerc, maire de la cour des tenants du Val-Benoît, compteur et receveur du ValBenoît, 98. - Voir VerT-Cheval.

Chilianus, voir KILIEN.

Chimay (Jean de) ou Mouton, cha- 
noine de Saint-Materne, chapelain de Notre-Dame de Heigne, 66.

Chinstrée (Gilles de), chanoine de Sainte-Croix, 64.

Christian, Cristianus, voir CortemBaCh, GutPhania, WeERdt.

Christine, Cristina (sainte), 80.

Chr ysogone, Crisogonus (saint), 101.

Cill Dara, voir Kildare.

Ciney (Namur, Dinant, Ciney), chapitre. - Abbé, voir СНавот.

Clemens, voir CléMEnT.

Clément I ${ }^{e r}$, Clemens (saint), pape, 100.

Clet, Cletus (saint), pape, 77.

Cockerill, place de Liège, 63.

Cokiers (Arnoul de), chanoine de Saint-Materne, 37, 73.

Cokins (Jacques le) ou Coquina, chanoine et doyen de Notre-Dame de Huy, 67.

Colardus, voir COLLARD.

Colin, Collinus, voir Worouz.

Collard, Colardus, voir Datonghe, MEEFFE.

Collinus, voir CoLIN.

Cologne (R.F.A., Rhénanie-Westphalie). -- Diocèse, 12. - Évêque, voir MATERNE (saint).

Colomannus, compagnon de saint Kilien, 77.

Colomban, Columbanus (saint), 100.

Columbanus, voir Colomban.

Condé (Jean de), prévôt de SaintLambert, 63.

Conrad, évêque de Pơrto, légat apostolique, 12.

CoppIN (Gérard), neveu de Jean Lathomé, 83.

Coquina, voir CoKINs.

Cortembach (Christian de), chanoine de Saint-Materne, 91. - (Gérard de), chanoine d'Aix-la-Chapelle, de Saint-Denis, de Saint-Lambert, abbé de Visé, 91.
Cortenbach, voir CORTEMBach.

Couronnés (les quatre saints), 24, 96.

CréPIN, Crispinus (saint), 92.

CrépINIEN, Crispinianus (saint), 92.

Crescence (sainte), 68.

Crisogonus, voir ChRysogone.

Cristianus, voir Christian.

Cristina, voir Christine.

Croisiers, couvent de Liège, 84.

Cunibert, Cunibertus (saint), 97.

CYRIN (saint), 68.

Damase $I^{\mathrm{er}}$, Damasus (saint), pape, 104.

Damasus, voir DAmase.

Datonghe (Collard), 61.

Daules (Gilles de), chanoine de SaintMaterne, 82.

DenIS, Dyonisius (saint), 24.

Digma (Jean), Dyck ou d'Eyck, chanoine de Saint-Materne, chapelain et receveur de l'Aumône de SaintLambert, 90.

Dinant (Namur, Dinant, Dinant). Chapitre, voir NoTrE-DAME.

Dinant (Florent de), chanoine de Saint-Materne, 86.

Domicianus, voir DoMITIEN.

Domitien, Domicianus (saint), 60.

DOMPIERRE (Godescalc de), chanoine de Saint-Lambert, prévôt de SaintBarthélemy, 79.

Dompire, voir DOMPIERRE.

Dormants (les sept), 24, 72.

Durant (Guillaume), évêque de Mende, 25, 26.

Dyck, voir Digma.

Dyonisius, voir DENIS.

Egidius, voir GiLLes.

ÉLEUTHÈRE (saint), compagnon de saint Denis, 88.

Élie, Helya, voir BoulLlon.

Eligius, voir ÉLoI.

ÉLISABETH, Elizabeth, Elyzabeth 
(sainte), 23, 75. - ÉLISABETH DE HONGRIE (sainte), 100. - Fille de Hanetay, 106. - Voir Purrs.

ÉLoI, Eligius (saint), 102.

Elyzabeth, voir ÉLISABETH.

Émichon, doyen de Notre-Dame de Huy, 67.

EMPIRE GERMANIQUE, 2.

ENGis, Engys (Liège, Huy, Engis), 47.

Engys, voir ENGIS.

ENoc, chanoine de Saint-Materne, 53.

Ensivaiz, voir ENZIVAUX.

Enzivaux (Jean de), chanoine de Saint-Materne et de la PetiteTable, 57, 78.

ÉPIMAQue, Epymachus (saint), 60.

EPPES (Jean d'), archidiacre de Brabant, prévôt de Saint-Lambert, 66. Epymachus, voir ÉPIMAQUE.

ÉTIENNE, Stephanus (saint), 34, 105.

EUGÈNE, Eugenius (saint), 99. - IV, pape, 70.

Eugenius, voir EugÈNE.

EusìBE, Eusebius (saint), 80.

EustaCHE, Eustacius, Eustatius, Stassines, Stassinus (saint), 24, 95. voir Langdris, Stasson.

Eustacius, voir EustaCHE.

Eustatius, voir EUSTACHE.

ÈvE, Eva, femme, 40.

Eventius (saint), 24, 59.

Evrard, abbé de Saint-Mathias de Trèves, 13.

Eyck, voir Digma.

FABRI (Nicolas), chanoine de SaintMaterne, 78, 85.

Fehe, voir FexhE.

Felicitas, voir FéLICITÉ.

FÉLICItÉ, Felicitas (sainte), fêtée avec sainte Perpétue, 46. - (sainte), mère des sept frères martyrs, 77 , 100.

FÉliCole, Felicula (saint), 24, 42.

Félicien, Felitianus (saint), 67.
FÉLIX (saint), 85.

FÉronstrée, rue de Liège, 98.

Feuillien, Foillanus (saint), 94.

Fexhe, voir FeXHE-SLIns, Sohe, SOXHE.

FeXhe (Jean de), bourgeois de Tongres, 39. - (Jean de), fruitier, 39.

Fexhe-Slins, Fehe, Fexhe (Liège, Liège, Juprelle), 48, 78. - Lieudit, voir SoXHE.

FichON, voir BERMONBĖCHE.

Ficon, voir FICHON.

Fies, voir Fize.

Fiez, voir FIZE-LE-MarsaL.

Fiez (Willermus de), chanoine de Saint-Materne, 94.

Fıze, famille d'ecclésiastiques liégeois, 28. - (Amel de), collecteur apostolique dans le diocèse de Liège, chanoine et doyen de Saint-Denis, chanoine de Saint-Materne, de Notre-Dame de Tongres, official du prévôt Jean de Condé, 63. (Antoine de), chanoine de NotreDame de Tongres, de Saint-Lambert, de Saint-Jean l'Évangéliste, prévôt de Saint-Denis et de SainteCroix, official, 30, 35, 54. - (Gilles de), chanoine de SaintMaterne, 35, 55. -- (Gilles de), chapelain de Saint-Lambert, 35. - (Gilles de), chantre de NotreDame de Tongres, 35. - (Gilles de), frère des frères Antoine et Jean de Fize, lui-même, chapelain de Saint-Lambert et chanoine de Saint-Denis, 35. - (Jean de), chanoine de Saint-Jean l'Évangéliste, de Saint-Lambert, 35, 61. - (Jean de), chanoine de Saint-Pierre, 61.

Fize-le-Marsal, Fiez, Fyes (Liège, Waremrne, Crisnée), 55, 83, 84.

FLoRE (Joachim de), mystique italien, 20.

FLOREBERT, Florebertus (saint), 57. 
Florennes (Namur, Philippeville, Philippeville). - Chapelain, voir SART.

FLORENT, Florentius, maître, chanoine de Sainte-Croix, 86. - Voir DinANT.

Florentius, voir FLORENT.

Flox, voir FLOXHE.

Floxhe, Flox, lieu-dit à Vottem, 89.

Foillanus, voir FeuiLlien.

Fosses-la-Ville (Namur, Namur,

Fosses-la-Ville). - Chapitre, voir

SAINT-FEUILLIEN.

FrédÉgAND, Fredegandus (saint), 79.

Freris, voir VREREN.

Froidmont, (Namur, Namur, Jemeppe-sur-Sambre). - Seigneur, voir SART.

Frongnut (Gilles dit le), clerc de

Liège, chanoine de Saint-Materne, 105.

Frongut, voir FRONGNUT.

Fyes, voir Fize-LE-MARSAL.

GaLl, Gallus (saint), 89.

Gallican, Gallicanus (saint), 72.

Galterus, voir GAUTHIER.

Gaufridus, voir GAUFROID.

GAUFroID, Gaufridus, voir RUPIN.

GAUTHIER, Galterus, Walterus, maître, 68. - Voir Chauvency, HesDIN, MOORSELE.

Gélase, Gelasius (saint), 100.

Gelasius, voir GÉLASE.

Gelenus, voir GHISLAIN.

GELINDEN, Glendis, Glenes, Glins

[Limbourg, Hasselt, Saint-Trond

(Gelmen)], 12, 14, 63, 69, 84.

Gembluz (Jean de), dit del Sohe de

Fehe, 39.

Genefe, voir JENEFFE-EN-HESBAYE.

Geneffe, voir JENEFFE-EN-HESBAYE.

GENÈse, Genesius (saint), 84.

GengulPhe, Gengulphus (saint), 23, 60.
Georges, Georgius (saint), 56.

GÉRARD, Gerardus, vicaire de Villersl'Évêque, 89. - Voir Bomal, CHABOt, COPPIN, CoRTEMbach, MELTER, SART.

Gerardria, voir GÉRARDRIE.

GÉrARDRIE, Gerardria, rue et lieu-dit de Liège, 13, 57, 90, 91.

GÉrÉon, Gereo (saint), 23, 24, 88.

Germain, Germanus (saint), 63.

Germanus, voir GERMAIN.

Gertrude, Gertrudis (sainte), 49.

GervaIs, Gervasus (saint), 69.

Gervasus, voir GeRvaIs.

GHISLaIN, Gelenus, voir SART.

GILlES, Egidius (saint), 86. - Chanoine de Saint-Materne, 63. Voir Bermonbèche, Chinstrée, Daules, Fize, Frongnut, Gilorin(I), Presles, Rosettes, SainteMarie, Vinalmont.

Gilles (Jean), chanoine de Paris, prévôt de Saint-Lambert, cardinaldiacre au titre des Saints-Cosmeet-Damien, 28, 74.

Gilloriro, voit Gilorin(I).

GILORIN(I) (Gilles) ou Gilloriro, chanoine de Saint-Materne, 39.

Glendis, voir GELINDEN.

Glenes, voir GELINDEN.

Glins, voir GELINDEN.

Glons (Bastien de), chanoine de Saint-Materne, 89.

Gluns, voir GLons.

GOAR (saint), 76.

Gobert, Gobertus, voir SAINT-SERVAIS.

Goblion, Goblio, chanoine de SaintMaterne, 92.

GODEFROID, voir BOUVIGNES.

Godescalc, Godescalcus, voir DoMPIERRE.

Goffardus, voir THIERTEMPS.

GonDon, Gondulphus (saint), 79.

Gondulphus, voir GoNDoN. 
Gordianus, voir GoRDIEN.

GoRDIEN, Gordianus (saint), 60.

GréGoIre I $^{\text {er }}$ le Grand, Gregorius (saint), pape, 48. - IX, pape, 13.

GruCHEIR (Nicolas), chanoine de Saint-Materne, 92.

Guido, voir VIT.

Guillaume, Wilhelmus, voir Buten, Durand, Heers, Ryckel, SCheVART, STUT, WAVERA.

Guillemins, couvent de Liège, 83.

GutPhania (Christian de), chapelain de l'Église de Liège, 106.

GuY, évêque de Palestrina, légat pontifical, 11.

Habundus, Abundus (saint), 41, 84.

HADELIN, Hadelinus (saint), abbé de Celles, 23, 39.

HaEbscap (Yvan de), chanoine de Saint-Materne, 84.

HAENSANGH (Jean), chanoine de Notre-Dame de Tongres, de Sainte-Croix, de Saint-Lambert, scelleur, coûtre de Notre-Dame de Dinant, abbé séculier de Thuin, 23,45 .

Haensank, voir HAENSANGH.

HALKEN, voir ALKEN.

Hallois (Henri de), chanoine de Saint-Lambert, 75.

Haloes, voir HaLloIs.

HaNeTAY, 106.

Hanezanc, voir HAENSANGH.

Hansange, voir HEANSANGH.

Harstapel, voir HERSTAPPE.

Harstaple, voir HeRsTAPPE.

Harstappe, voir HeRSTAPPE.

Haute-SAuveniÈre, rue de Liège, 64.

Hebscaep, voir HAEBSCAP.

HeERS (Guillaume de), chanoine de

Saint-Materne, 83, 95. - (Jean de), sous-diacre, 41 .

Heigne (Hainaut, Charleroi, Jumet).

- Chapelain, voir ChIMAY.
Hélie, voir BouIllon.

Hélyas, voir Bouillon.

Helya, voir ELIE.

Hemricourt (Jean de), chanoine de

Saint-Materne, 87. - (Jean-Thomas de), chanoine de Saint-Denis, 87.

Henri $I^{\text {er }}$ de Verdun, évêque de Liège, 23. - Prêtre, 54. - Voir Alken, Hallois, Laecman, Oneal, PasSET, Pinte, Teghelen.

Herber T, Herbertus, voir AIx.

Hereneus, Hiereneus, Hireneus, Ireneus (saint), 24, 41, 84.

Herman, Hermannus, voir Blehen, Holongnele, XANTEN.

Hermannus, voir HERMAN.

Hermès (saint), 85.

HerstapPe, Harstapel, Harstaple, Harstappe (Limbourg, Tongres, Tongres), 47, 68, 80 .

Hers, voir HeERs.

Hesbaye, région de Belgique, 28.

HesDin (Gauthier de), chanoine de Saint-Materne, 53.

Hiereneus, voir Hereneus, Ireneus.

HIPPOLYTE, voir HYPPOLITE.

Hireneus, voir Hereneus, Ireneus.

Hollande (Arnoul de), chanoine de Saint-Lambert, 94.

Hollant, voir Hollande.

Holongnele (Herman de), miles, 84. HoNORIUS III, pape, 14.

Horion-HozÉmont (Liège, Liège, Grâce-Hollogne). - Dépendance, voir LEXHY.

HORNES, vOir THIERRY.

Horpale, voir HoR PMAAL.

HorPMAAL, Horpale [Limbourg, Tongres, Heks (Heers)], 54, 64, 94.

HubERT, Hubertus (saint), 23, 95.

Hugues II de Pierrepont, Hugo, évêque de Liège, $9,10,11,12,22,28$, 53.

Huy (Liège, Huy, Huy), 12. - Chapi- 
tre, voir Notre-DaME. - Voir Malechair.

HUYGHEBAERT (dom Nicolas), historien belge, 18.

HyPPOLITE, Ypolitus (saint), 24, 80.

IDE, Yda, voir PuTEo.

IGNACE, Ignatius (saint), 38.

Ignatius, voir IGNACE.

Île (André d'), chanoine de SaintMaterne, 41.

INNOCENT VII, pape, 74.

INNOCENTS, Innocentes (saints), 34, 106.

Insula, voir ÎLE.

Ireneus, voir Hereneus.

IRLANDE. - Monastère, voir KIL-

DARE.

Iuvenalis, voir JUVÉNAL.

IvaN, Iwanus, 83.

Iwanus, voir IVAN.

Jacinctus (saint), 24, 41.

Jacobus, voir JACQUES.

JACQUES le Mineur, Jacobus (saint),

59. - Voir Bocheroul, CAPElle,

Cokins, Looz, Lovelde, MaleChaIR, SART, UGREES.

JASPAR, Jasper, voir MORIALMÉ.

JEAN-BAPTISTE (saint), 72, 74, 85.

JeAN Chrysostome (saint), 36.

JEAN L'ÉvaNGÉLISTE (saint), 34, 106.

JEAN, Johannes (saint), frère de Paul,

72. - JEAN DEVANT LA PORTE D'ARSEYN (saint), 60. - II d'Eppes, évêque de Liège (auparavant prévôt de Saint-Lambert), 12, 13, 14. - VII de Heinsberg, évêque de Liège, 70. - XXI, pape, 12. Voir AIx, Alleur, Bourguignon, Bouvignes, Bruges, Chimay, Conde, Digma, Enzivaux, Fize, Gembluz, Gilles, Haensangh, HeERs, Hemricourt, JupILLE, LABELle, LATHOMÉ, LeONARD,
Longue Rue, Marcella, MarliNes, Moregny, Nazareyo, NefFe, Perwez, Pexhon, Pirlin, Preit, Salm, Soxhe, Tasseron, WellyNES.

JenEFFe-En-Hesbaye, Genefe, Geneffe (Liège, Waremme, Donceel), 56, 60.

JOACHIM, vOir FLORE.

JosSE, voir BOURGUIGNON.

Juda, voir JUDE.

JuDE, Juda (saint), 93.

Juliana, voir JULIENNE.

JULIENNE, Juliana (sainte), 42.

JuPILLE (Jean de), chanoine de SaintLambert, 71.

JuvÉNAL, Iuvenalis (saint), 24, 59.

Katherina, voir CATHERINE.

KemeXhe (Liège, Waremme, Crisnée), $72,106$.

Kildare, Clll Dara, monastère irlandais, 38.

Kilian, voir KILLIEN.

Killien, Kylianus (saint), 23, 24, 77.

Killanus, voir KILIEN.

KirPeCH (Pierre de), chanoine de

Saint-Lambert, de Saint-Paul, de

Saint-Séverin de Cologne, 23, 48.

Kirpeck, voir KIRPECH.

KoKIN (Pierre), chanoine de Saint-

Materne, tenant de la cour de

Saint-Denis et de Saint-Lambert, 50.

Kylianus, voir KILIEN.

LABELLE (Jean), chanoine de SaintMaterne, chapelain de Saint-Lambert, 70 .

LAECMan (Henri), chanoine de SaintMaterne, 41, 95.

LAHAYE (Léon), historien belge, 29.

LAMBERT, Lambertus (saint), évêque de Liège, 23, 89. - Voir OpHEERS, STAVELOT. 
Landris, voir LANGDRIS.

LANGDRIS (Eustache de), chanoine de Saint-Materne, 48.

LATHOMÉ (Jean) ou Lathomi, chanoine de Sainte-Croix, de la PetiteTable, 83.

LAURENT, Laurentius (saint), 81.

Laurentius, voir LAURENT.

LEMAÎTRE (Jean-Loup), historien français, 18, 19.

LEON I ${ }^{\mathrm{er}}$, Leo (saint), pape, 53. - II

(saint), pape, 73 .

LÉONARD (saint), 96.

LEONARD (Jean) ou Lynar, chanoine de Saint-Materne, 95.

Leonardi, voir LEONARD.

LEXHY, Lexi, dépendance de HorionHozémont, 78.

Lexi, voir LEXHY.

Lieerses, voir LIERs.

LIÈGE. - Abbayes, voir ROBERMONT, SAINT-JacQues, SaInT-LauRent, VAL-BENoît. - Archidiacres, voir Chauvency, EPPes, Ludol.PHE, Moregny. - Cathédrale, voir SAINT-LAMBert. - Chancelier de l'évêque, voir SART. - Chapitres secondaires de la cathédrale, voir Saint-Materne, Petite-Table. Collecteur de la chambre apostolique pour le diocèse, voir SART. Collégiales, voir SAINT-BARTHÉLEMY, SAINT-Denis, SAINT-JEAN L'Évangéliste, SaInt-Paul, Saint-Pierre, Sainte-Croix. Couvents, voir Bons-EnFants, Croisiers, Guillemins. - Diocèse, $1,2,12,23,70$; collecteur apostolique, 63. - Églises, voir SAINT-Christophe, SAINT-Servais, Saint-Severin, Saint-Thomas, SaINTE-MARIE-MAdeleine. - Élu, voir THIERry dE HORNES ou DE Perwez. -- Évêques, voir Albert $I^{\text {er }}$ DE Louvain, Henri $I^{\text {er }}$ de Verdun, Hugues II de PierrePONT, Jean II D'Eppes, JeAn VII DE HEINSBERG, LAMBERT (SAINT). - Hôpital, voir SaINT-MathIEUÀ-LA-CHAî́NE. - Lieux-dits, voir GÉrardrie, Vivegnis. - Maisons, voir BRESINE Bastin, TroisPierres, Vignerons. - Official, voir FIzl. - Paroisses, voir Saint-Servais, Saint-Severin. Places, voir Cockerill, RéPUbliQUE Française. - Principauté, 3. - Quartiers, voir Outremeuse, Sauvenière, Torrent. - Rues, voir Basse-Sauvenière, ChaPELle-DES-Clercs, FÉronstrée, Gérardre, Haute-Sauvenière, Pont, Pont d'Avroy, Régence, UnIVERSITÉ. - Scelleur, voir HAENSANGH.

LIÈGE (Alain de), chanoine de NotreDame de Tongres, secrétaire de l'archidiacre et coûtre de SaintLambert, Jean d'Eppes, 66.

LIERS, Lieerses (Liège, Liège, Herstal), 99.

Limont (Liège, Waremme, Donceel), 93.

LIN, Linus (saint), pape, 101.

Linus, voir LiN.

LizIEUX (France, Calvados). - Archidiacre, voir TEXTOR.

LOMBARDIE, région italienne, 58.

Longue Rue (Jean dit de), chanoine de Saint-Materne, 88.

LoOz (Jacques de), chanoine de SaintLambert, prévôt de Saint-Denis, 58.

Lovelde (Jacques de), chanoine de Saint-Lambert, 42.

LuC, Luca(s) (saint), 90.

LUDOLPHE (ou Lydulphe), archidiacre, 13.

Lydulphe, voir LUDOLPHE.

Lynar, voir LEONARD. 
MAASeIK (Limbourg, Maaseik, Maaseik). - Chapitre, voir NOTREDAME.

MaAstricht (Pays-Bas, Limbourg). -Chapitre, voir SaINT-Servais.

Machinia, voir MARLINES.

Machlinia, voir MARLINES.

Magnus (saint), 82.

MAIRSU, 49.

Malechair (Jacques ou Jakemars) de Huy, chanoine de la Petite-Table, de Saint-Materne, de Saint-Paul, chanoine et chantre de NotreDame de Huy, 56.

Malines (Anvers, Malines, Malines). - Chapitre, voir SAINT-RombaUt. Malle (Olivier de), chanoine de Saint-Materne, 42.

MARC, Marchus, Marcus, 54. (saint), évangéliste, 57. - (saint), fêté avec Marcellin, 69. - (saint), pape, 88. - Chanoine de SaintMaterne, 99.

MARCEL, Marcellus (saint), 88.

Marcella (Jean de), chanoine de Saint-Materne, 54. - Ses parents, 54.

Marcellianus, voir MARCELLIN.

MARCELlin, Marcellianus, Marcellinus (saint), fêté avec saint Marc, 69. - (saint), fềté avec saint Pierre, 65.

Marchus, voir MARC.

MarciUs (saint), 24, 42.

Margareta, voir MARGUERITE.

MARGUERITE, Margareta (sainte), 78.

Maria Magdalena, voir MARIE-MADELEINE.

Marie, Maria, servante de Jean le Bourguignon, 48.

MARIE ad martyres, Maria (sainte), 61.

MARIE L'ÉGYPTIENNE, Maria (sainte), 52.
MARIE-MAdeleine, Maria Magdalena (sainte), 80.

Marlines (Jean de), chanoine de Saint-Lambert et de Saint-Paul, 46,100 .

Maroie, voir Mont.

MARTIN, Martinus (saint), 75, 97. $\mathrm{I}^{\text {er }}$ (saint), pape, 97. - Voir VERTCheval.

Martinianus, voir MARTINIEN.

MARTINIEN, Martinianus (saint), 75 .

Martyrs (les sept frères), 24, 77.

Materne (saint), évêque de Cologne, évêque de Trèves (?), 11, 12, 13.

Mathia, voir MAT(T)HIAS.

Mat(t)hias, Mathia (saint), 44.

Maures (saints), 24, 89.

Maximin, Maximinus (saint), fêté avec saints Tiburce et Valerien, 54. - (saint), 64.

Maximinus, voir MAXIMIN.

MECHELEN-Bovelingen, Marlines [Limbourg, Tongres, Heks (Heers)], 69.

Medardus, voir MÉDARD.

MÉDARD, Medardus (saint), 67.

MEEFFE (Collard de), chanoine de Saint-Materne, 21, 104.

Melter (Gérard), chanoine de SaintMaterne, 90.

MENDE (France, Lozère). - Évêque, voir Durant.

Menne, Menna (saint), 97.

Milmort, Miremorte (Liège, Liège, Herstal), 71.

Miremorte, voir MILMORT.

MODESTE (saint), 68.

MonéGonde, Monegundis (sainte), 74. Monegundis, voir MONÉGONDE.

Monon, Mono (saint), 90.

Mont (Maroie de), épouse de Jean delle Soxhe de Fexhe, 39.

Monulphe, Monulphus (saint), 79.

MoOrSEle (Gauthier de) chanoine de Saint-Materne, 28, 33, 59, 86. 
Morealmes, voir MORIALMÉ.

MOREGNY (Jean de), chanoine et doyen de Saint-Lambert, archidiacre, prévôt de Saint-Jean l'Évangéliste, écolâtre de Saint-Denis, 56, 98.

Morialmé (Jaspar de), chanoine de

Saint-Materne, 82.

Morseilh, voir MoORSELE.

Morselhe, voir MOORSELE.

Mouthon, voir MouToN.

Mouton, voir Chimay.

Moylant (Antoine de), 74.

Museler (Antoine), chanoine de

Saint-Materne, 103.

Muselere, voir MUSELER.

NABOR (saint), 68.

NAMUR (Namur, Namur, Namur), 12.

- Diocèse, 12.

Narciscus, voir NARCISSE.

NARCISSE, Narciscus (saint), 93.

NAveroule, Nawerulle, dépendance

de Villers-l'Évêque, 106.

Nawerulle, voir NAVEROULE.

NAZAIRE (saint), 68.

Nazar, voir NAZAIRE.

NAzAREYo (Jean de), chanoine de

Saint-Materne, 87.

NefFe (Jean delle), 39.

NÉRÉE, Nereus (saint), 61.

Nereus, voir NÉRÉE.

NézIGNan (France, Hérault), église.

- Recteur, voir TeXTOR.

Nicholaus, voir NicOLAS.

Nichomedis, voir NicomèDE.

Nicolas, Nicholaus, Nicolaus (saint),

103. - Voir Fabri, GrucheIR,

TURRE.

Nicolaus, voir Nicolas.

NicOMÈDE, Nichomedis (saint), 65 .

Nivelles (Brabant, Nivelles, Nivel-

les). - Chapitre, voir SAINT-PAUL.

Notre-Dame, chapitre d'Aix-la-Cha-

pelle. - Chanoine, voir SART.
Notre-DAME, chapitre de Dinant. Coûtre, voir HAENSANGH.

Notre-Dame, chapitre de Huy. Chanoines, voir CoKIns, MaleCHAIR. - Chantre, voir MaLECHAIR. - Doyens, 28; voir CoKIns, Emichon, Xanten.

Notre-DAme, chapitre de Maaseik. - Abbé séculier, voir BERMONBÈCHE.

Notre-Dame, chapitre de Tongres, 12. - Chanoines, voir Fize, Haensangh, Liège. - Chantre, voir FIZE.

Notre-Dame, voir SaINTE-Marie.

Notre-Dame (Jean de), chanoine de la Petite-Table, 67. - (Jean de), clerc de Liège, 67.

Notre-Dame-AuX-Fonts, église de Liège, 10, 11, 101. - Abbé, 13, voir Chauvency. - Chanoines (chapitre, prébendes), 10.

NuCERIo (Antoine de), 61.

Ochain, voir Chabot.

ODE, Oda, Ooda (sainte), 24, 92. Mère de maître Élie, 28, 50 .

ODEuR, Odor (Liège, Waremme, Crisnée), 68, 106.

Odor, voir ODEUR.

OfFeT (Simon), chanoine de SaintMaterne, 44.

OHEY (Namur, Namur, Namur), 70.

Okires, voir COKIERS.

OlIVIER, voir MALLE.

ONEAL (Henri), chanoine de SaintMaterne, 28, 37, 38, 95. - - Ses parents, 38. - (Henri), père d'Henri Oneal, chanoine de SaintMaterne, 38.

ONZE Mille Vierges, 23, 91.

Op Here, voir OPHEERS.

Opeyum, voir OUPEYE.

OpHEERS (Lambert de), chanoine de 
Saint-Materne et de Saint-Jean l'Évangéliste, 34.

Oteey, voir OTHÉE.

OTHÉ, Oteey, Othee, Othey (Liège,

Liège, Awans), 41, 48, 58, 62.

Ohee, voir OTHÉE.

Othey, voir OTHÉE.

Othmarus, voir OTMAR.

Otmannus, voir Totmannus.

OtMar, Othmarus (saint), 99.

OUPEYE, Opeyum (Liège, Liège, Oupeye), 46.

Outremeuse, ultra Mosam, quartier de Liège, 54, 94.

OvIDE, poète latin, 25.

Oxhen, voir OCHAIN.

Palestrina (Italie, Latium, pr. Rome). - Évêque, voir GuY.

Pancrace (saint), 61 .

PARIS (France, Paris). - Chanoine, voir GiLles.

Passet (Henri), chapelain de SaintLambert, 82.

Paul, Paulus (saint), 73. - (saint), frère de Jean, 72.

Paulin, Paulinus (saint), 71. (saint), 85.

Pepenges, voir Pepingen.

Pepingen, Pepenges [Limbourg, Tongres, Heers (Mechelen-Bovelingen)], 66.

Perpétue, Perpetua (sainte), 46.

Per pète, Perpetuus (saint), 96.

Perwez (Jean de), chanoine de SaintMaterne, 47. - Voir THIERRY.

Petite-Table, chapitre secondaire de la cathédrale de Liège. - Chanoines, 2, 28, 98; voir Albert, ARnould, EnzivauX, LATHomé, MALECHAIR, Notre-DaME.

PÉtronille, Petronilla (sainte), 64.

Petrus, voir PIERRE.

Pexhon (Jean), chanoine de SaintMaterne, de Saint-Pierre, 81.
PhILIBERT, Philibertus (saint), 82.

Philippe, Philippus (saint), 59.

Pierre, Petrus (saint), 12, 73. (saint), fềté avec saint Marcellin, 65. - (saint), évêque, 101. Pierre de Vérone (saint), 58. Voir Bourguignon, Cheval, KIRPECH, KoKIN.

PINTE (André), Chapelain, 78. -

(Henri), chapelain de Saint-Denis et de Saint-Lambert, employé du Val-Benoît, 46.

PIrLIN (Jean) de Villers-l'Évêque, 41. PISE (Italie, Toscane, pr. Pise), 74.

Poitilers (France, Vienne). - Bénéficier, voir RuPIN.

Polycarpe, Policarpus (saint), 36.

Ponce, Pontius, voir Textor.

PonCELET (Édouard), historien belge, 9, 10, 11.

Pons Avroti, voir Pont D'Avroy.

PonT, rue de Liège, 98.

Pont D'Avroy, Pons Avroti, rue de Liège, 84, 93.

Pontius, voir PONCE.

PorTo. - Évêque, voir Conrad.

Poucet, Pucey, Puchey (Liège, Waremme, Hannut), 37, 56, 67.

Pousset, Pucey, Puchey (Liège,

Waremme, Remicourt), 37, 56, 67.

Prato, voir PreIT.

PraXèDE, Praxedis (sainte), 79.

PREgt, voir Prix.

Preit (Jean de), chanoine de Saint-

Materne, de Saint-Servais de

Maastricht, 76.

Prella, voir PRESLes.

Presles (Gilles de), chanoine de SaintMaterne, 97.

Prey, voir PRIX.

Priest, voir PRIX.

Prime, Primus (saint), 67.

Priscus (saint), 86.

Privat, Privatus (saint), 82.

PrIX, Proiectus (saint), 36. 
Processe, Processus (saint), 75.

Processus, voir PROCESSE.

Proiectus, voir PrIX.

Protais, Prothasus (saint), 69.

Prothasus, voir Protais.

Psalmis, voir SALM.

Pucey, voir Poucet, Pousset.

Puche, voir Purts.

Puchey, voir Poucet, Pousset.

Puiche, voir PuITs.

PuITs (Élisabeth du), épouse de Thonard du Puits d'Alleur, 46. (Thonard du) d'Alleur, homme allodial, 46, 47 .

Puteo (Ide de), mère de Henri Oneal, 95.

Quellien, voir KILIEN.

Quentrs, Quintinus (saint), 94.

Quintinus, voir QUENTIN.

RAOul, Rodulphus, chanoine de SaintMaterne, 103.

RéGENCE, rue de Liège, 63.

ReIMs (France, Marne), 40.

REMACLE, Remaclus (saint), 87.

Renerus, voir RENIER, RÉNIER.

RENIER, Renerus, voir VILHE.

RÉNIER, Renerus, voir WASAR.

RÉPUBLIQUE FRANÇAISE, place de Liège, 63.

Richel, voir RYCKEL.

Richelle, voir RYCKEL.

RoBERMONT, abbaye de Liège, 93 . Chapelain, voir SART.

Rodulphus, voir RAOUL.

Rombaldus, voir Rumoldus.

Rome (Italie, Latium, pr. Rome).

Concile, 12.

Ros(s)ETtes (Gilles de), chanoine de Saint-Materne, 36.

RoTARII (Thierry), chanoine de SaintMaterne, 99.

Roussetes, voir Ro(s)SETTES.

RuF, Rufus (saint), 85.
Rumoldus (saint), 93.

RUPIN (Gaufroid), chanoine et vicedoyen de Saint-Lambert, bénéficier dans la région de Poitiers, 23, 42.

Russon (Limbourg, Tongres, Tongres), chapitre. - Chanoine, voir Stavelot.

RUSTIQUE (saint), compagnon de saint Denis, 88.

RYCKel (Guillaume de), chanoine de Saint-Materne, 52, 75.

SABINE, Sabina (sainte), 85.

Sabuletum, voir SAUVENiÈRE.

SaInt-Barthélemy, collégiale de Liège. - Chanoine, voir SART. -... Prévôt, voir DOMPIERRE.

SaInT-Chair (Jean de), chanoine de Saint-Materne, 67. - Voir Cokins.

Saint-Chrisrophe, église de Liège, 70.

SAINT-Denis, collégiale de Liège, 70 . - Chanoines, voir BERMONBÈCHE, Cortembach, Fize, Hemricourt, SART. - Chapelains, voir BERMONBÈCHE, PINTE. - Doyens, 28 ; voir Chabot, Fize, Sart. - Écolâtre, voir MoRegny. - Prévôts, voir FIZE, Looz. - Tenant de la cour, voir KoKIN.

Saint-Feuillien, chapitre de Fosses.

- Prévôt, voir Chauvency.

SAINT-GoAR (R.F.A., Rhénanie-Palatinat), chapitre. - Chanoine, voir SALM.

Sant-Hadelin, chapitre de Visé. Abbés séculiers, voir CORTEMBACH, XANTEN.

SAINT-JACQUES, abbaye de Liège, 14, $16,17$.

SAINT-JEAN L'Évangéliste, collégiale de Liège, 76. - Chanoines, voir Fize, OpheERs, Wavera. - Cha- 
pitre, 92. - Prévôt, 92; voir MOREGNY.

SAINT-LAMBERT, cathédrale de Liège, $2,9,11,34,37,46,53,58,61,91$, 101. - Bénéficiers, 2, 11, 98. Chanoines, 2, 3, 9, 11, 12, 22, 28 ; voir Aix, BERMONBÈCHE, BOMAL, Boulllon, Broechem, Chabot, Cortembach, Dompierre, Fize, HaEnsangh, Hallois, Hollande, JuPille, KirPech, Looz, Lovelde, Marlines, Moregny, Rupin, SART, TeXtor, XANTEN. Chapelains, voir DIgMA, FIzE, Labelle, Passet, Pinte. - Chapitre, 13, 19, 51. - Cloîtres, 101. Compteur, voir Broechem. Confrérie, voir SaInT-Luc. Doyens, 13, 28 ; voir Chauvency, MOREGNY. - Écolâtre, voir Chauvency. - Prévôts, 13 ; voir CONDÉ (son official, voir FIZE), Eppes, Gilles, Jean II d'EpPes. Receveur de la Grande Compterie, voir Wellynes. - Receveur de l'Aumône, voir Digma. - Receveur, voir XANTEN. - Tenant de la cour jurée, voir KoKIN. - Vicedoyens, voir RuPIN, Xanten.

SaInT-LaUrent, abbaye de Liège, 23. SAINT-Luc, confrérie de la cathédrale de Liège, 98.

SaInT-Materne, chapitre secondaire de la cathédrale de Liège. - Chanoines, voir Arx, Alken, Alleur, BANOW, BleHEN, Bocheroul, Bouchout, Bourguignon, Bréda, Bruges, Bucke, Buten, Capelle, Cokiers, Cortembach, Daules, Digma, Dinant, Enoch, Enzivaux, Fabri, Fiez, Fize, Frongnut, Gilles, Gilorin(I), Glons, Goblion, Grucheir, HaEbscap, HeErs, Hemricourt, Hesdin, Île, KoKin, Labelle,
LAECMAN, LEONARD, LONGUE Rue, Malechair, Marcelle, Marc, Meeffe, Melter, MoorSele, Morialmé, Mouton, MuseLer, Nazareyo, Offet, Oneal, Opheers, Perwez, Pexhon, Preit, Presles, Raoul, Rotarit, RycKel, Saint-Chair, Sainte-Marie, Salm, Sart, Schevart, Stut, Theux, Vilhe, Wellynes. - Chapelain, voir TURRE. - Compteur, voir TEGHELEN.

SaInT-Mathias, abbaye de Trèves. Abbé, voir Evrard.

SaINT-Mathieu-À-LA-Cha îne, hôpital de Liège, 11, 101.

SaInT-Paul, collégiale de Liège, 78. - Chanoines, voir KIRPECH, Malechair, Marlines. - Prévôt, voir Chabot.

SaINT-PaUl, chapitre de Nivelles.

Chanoine, voir ChaBot.

SaINT-Pierre, collégiale de Liège. Cellérier, 35. - Chanoines, voir Bermonbèche, Fize, Pexhon, SART. - Coûtre, voir TeXtor. Prévôt, voir XANTEN.

SAINT-Rombaut, chapitre de Malines.

- Prévôt, voir Broechem.

SaInT-Servais, chapitre de Maastricht. - Chanoine, voir PreIT. Église et paroisse de liège, 35, 46. SaInT-Servais (Gobert de), presb., 96. SAINT-SEVERIN, paroisse et église de Liège, 98.

SAInT-SÉverin, chapitre de Cologne.

- Chanoine, voir KIRPECH.

SaINT-Thomas, église de Liège, 57.

SAINTE-CroIX, collégiale de Liège. --. Chanoines, voir Chinstrée, Florent, Haensangh, Lathomé. Chapelain, voir SART. - Doyen, 28 ; voir Bouvignes. - Prévôt, voir Fize.

SaINTE-Marie, voir Notre-Dame. 
SaINTE-Marie (Gilles) ou de NotreDame, chanoine de SaintMaterne, 30, 40.

Sainte-Marie-Madeleine, église de Liège, 82.

SAINTS-CoSme-ET-DAmien, titre cardinalice. - Voir Gilles.

Salm (Jean de), chanoine de SaintGoar et de Saint-Materne, 64.

Sancto Caro, voir SAINT-ChalR.

SART (Gérard de), chanoine de SaintMaterne, de Saint-Barthélemy, de Saint-Lambert, 70, 87. - (Gérard de), prêtre, chapelain de SainteCroix, 87. - (Ghislain de), chanoine de Saint-Materne, collecteur de la Chambre apostolique pour le diocèse de Liège, chanoine et doyen de Saint-Denis, de NotreDame d'Aix, chapelain de Florennes et de Robermont, seigneur de Froidmont, chanoine de SaintPierre, chancelier de l'évêque de Liège et du duc de Brabant, 70. (Ghislain de), 87. - (Jacques de), chanoine de Saint-Denis, de SaintMaterne, 104.

Saturnin, Saturninus (saint), et ses compagnons, 24, 102.

SAUVENIĖRE, Sabuletum, quartier de Liège, 54.

SCHEVART (Guillaume), chanoine de Saint-Materne, 62.

SCHOlastique, Scolastica (sainte), 41. Scolastica, voir ScholastiQUE.

Senari, voir NAZAIRE.

ServaIS, Servatius (saint), 23, 61.

Servatius, voir SERvaIS.

SÉVÈRE, Severus (saint), 100.

Severinus, voir SÉvERIN.

SÉvERIN, Severinus (saint), 92.

Severus, voir SÉvĖRE.

Silvester, voir SYLVESTRE.

SIMON, Symo (saint), 93. - Voir OFFET.
Simphorianus, voir SIMPHORIEN.

SIMPHORIEN, Simphorianus (saint), 83.

Sotheris (sainte), 41.

SoxhE, lieu-dit à Fexhe-Slins, 39.

Soxhe (Jean delle) de Fexhe, époux de

Maroie de Mont, 39, 48, 78.

Stabulis, voir Stavelot.

Stassines, voir EustaCHE.

Stassinus, voir EUSTACHE.

Stasson (Eustache), tailleur ou drapier, 49.

Stavelot (Lambert de), chanoine de Russon, 40, 69.

Stephanus, voir ÉTIENNE.

StUT (Guillaume), chanoine de SaintMaterne, 72.

SyLVESTRE I ${ }^{\text {er }}$, Silvester (saint), pape, 107.

Symo, voir SIMON.

TASSERON (Jean), 53.

Tectis, voir THEUX.

Teghelen (Henri de), compteur de Saint-Materne, 84.

Textor (Ponce), chanoine de SaintLambert, coûtre de Saint-Pierre, recteur de l'église paroissiale de Nézignan et archidiacre de Lizieux, 81.

Theodericus, voir THIERRY.

Theodolus (saint), 59.

THÉODORE, Theodorus (saint), 96.

Theux (Baudouin de), chanoine de Saint-Materne, 78, 85.

ThiERry de Hornes ou de Perwez, élu irrégulièrement à l'évêché de Liège, 43.

THIERRY, Theodericus, voir BUCKE, ROTARII.

THIERTEMPS (Goffardus), 68.

Thoma, voir THOMAs.

Thomas, Thoma (saint), 75, 105.

THOMAS BECKET, Thoma (saint), 106.

Thonard, Thonardus, voir PuIts.

THuin (Hainaut, Thuin, Thuin), cha- 
pitre. - Abbé séculier, voir HAENSANGH.

TrBurCE, Tyburtius (saint), 54.

TIMOTHÉE, Timotheus, Tymotheus (saint), fêté avec saint Simphorien, 83. - (saint), fêté avec saint Appolinaire, 83.

Timotheus, voir TimothéE.

TIRLEMONT (Brabant, Louvain, Tirlemont), chapitre. - Écolâtre, voir BoulLLoN.

TONGRES (Limbourg, Tongres, Tongres), 12. - Bourgeois, voir Fexhe. - Chapitre, voir Notre-DAME.

Toringium, voir TORRENT.

TORRENT (en), Toringium, quartier de Liège, 63, 65.

Totmannus, compagnon de saint Kilien, 77.

Totnanus, voir Totmannus.

TrÈves (R.F.A., Rhénanie-Palatinat).

- Abbaye, voir SaINT-Mathias.

- Diocèse, 12. - Évêque, voir MATERNE (saint) (?).

Trimeies (Baudouin de), presb., 79.

Trois-PIERRES, maison de Liège, 98.

TRUDON, Trudo (saint), 101.

TURRE (Nicolas de), chapelain de

Saint-Materne, 57.

Tyburtius, voir TIBURCE.

Tymotheus, voir TIMOTHÉE.

Ubertin, voir Casale.

UdALRIC, Ulricus (saint), 75.

UGREES (Jacques d'), presb., 71.

Ulricus, voir UDALRIC.

Ultra Mosam, voir OUTREMEUSE.

UNIVERSTIÉ, rue de Liège, 63.

URBAIN $I^{\text {er }}$, Urbanus (saint), pape, 62.

Urbanus, voir URBAIN.

Ursmarus, voir URSMER.

URSMER, Ursmarus (saint), 23, 55.

VAAST, Vedastus (saint), 40.

VALENTIN, Valentinus (saint), 42.
VALERIEN, Valeriamus (saint), 54.

VALERIUS (saint), 37.

VAL-BENoîT, abbaye de Liège. Abbesse, 39. - Compteur, voir Cheval. - Cour des tenants, 98. - Maire de la cour des tenants, voir Ver T-Cheval. - Mambour, voir Vert-Cheval. - Personnel, voir PiNTE. - Receveur, voir CHEVAL.

Vedastus, voir VAast.

Vert Cheval (Martin du), brasseur, mambour du Val-Benoît, 98.

VIGNeRons (maison des), maison de Liège, 98.

Vilari, voir VILLERS-L'Évitoue.

Vilarium Episcopi, voir VILlersL'ÉVÊQUE.

Vileri Episcopi, voir VILLRS-L'ÉvêQUE.

VILHE (Renier de), chanoine de SaintMaterne, 52, 75.

Villare, voir VILLERS-L'ÉvÊQUE.

Villari Episcopi, voir VILLERS-L'ÉvêQUE.

Villarium Episcopi, voir VILLERSL'ÉVÊQuE.

VILLERS-L'ÉvÊQue, Vilari, Vilarium Episcopi, Vileri Episcopi, Villare, Villari Episcopi, Villarium Episcopi (Liège, Liège, Awans), 11, 13, 14, $38,39,40,43,44,50,53,55,91,95$. - Dépendance, voir Naveroule. - Vicaire, voir GÉRARD. - Voir Pirlin.

Vinalmont (Gilles de), doyen de Saint-Denis, 70.

Vinetum, voir VIVEGNIS.

VISÉ (Liège, Liège, Visé), 39. - Chapitre, voir SAINT-HadeliN.

VIT, Vitus (saint), 68.

VitAL (saint), 24, 42.

Vitalis (saint), 58.

Vitus, voir ViT.

VOROUX-GOREUX, Vorous, Vorrus, 
Voruz, Woruz (Liège, Waremme, Fexhe-le-Haut-Clocher), 37, 63, $73,99,103$.

VoROUX-LEZ-LiERS, Vorous, Vorrus,

Voruz, Woruz (Liège, Liège, Juprelle), 37, 73, 99, 103.

Vorous, voir VOROUX-GOREUX, VOROUX-LEZ-LIERS.

Vorrus, voir VOROUX-GoreuX, VOROUX-LEZ-LIERS.

Voruz, voir VOROUX-GoreUX, VOROUX-LEZ-LIERS.

Voteme, voir VotTem.

Votemme, voir VoTtEM.

Votтем, Voteme, Votemme (Liège, Liège, Herstal), 53, 71. - Lieu-dit, voir Floxhe.

VREREN, Freris (Limbourg, Tongres, Tongres), 51.

WALBURGE, Walburgis (sainte), 59.

Waldetrudis, voir WAUDRU.

Walterus, voir GAUTHIER.

Wamon, voir WAMONT.

WAMONT, Wamon [Brabant, Louvain, Landen (Houtain-l'Évêque)], 36, 75.

Warnerus, voir WARNIER.

WARNIER, Warnerus, 66.

WASAR (Rénier, dit), moine, frère de

Willermus de Fiez, 94.

WAUDRU, Waldetrudis (sainte), 39.

WaVERA (Guillaume de), chanoine de Saint-Jean l'Évangéliste, 42.
WeErdt (Christian de), chanoine de Saint-Materne, 91.

Weert, voir WEERDT.

Wellynes (Jean de), chanoine de Saint-Materne, 97, 98. - (Jean de), clerc, homme de la cour allodiale, receveur de la Grande Compterie de Saint-Lambert, 97, 98.

Wido, voir VIT.

Wilhe, voir VILHE.

Wilhelmus, voir Guillaume.

Willebrordus, voir WILLIBRORD.

Willermus, voir Fiez.

Willibrord, Willebrordus (saint), 96.

Worouz (Colin de), 99.

Woruz, voir VOROUX-GOREUX, VOROUX-LEZ-LIERS.

XANTEN (Herman de), chanoine, vicedoyen et receveur de Saint-Lambert, prévôt de Saint-Pierre, doyen de Notre-Dame de Huy, abbé séculier de Visé, 28, 33, 38, 45, 51, $59,65,74,86,94,102$.

Yde, voir IDE.

Ypolitus, voir HyPPOLITE.

YVAN, Yvanus, voir HAEBSCAP.

Yvanus, voir YvaN.

Zanctis, voir XANTEN.

ZÉNON, Zeno (saint), 24, 42.

Zoticus (saint), 24, 41. 